UNIVERSIDADE DE BRASÍlIA

Departamento de Matemática

Curso de Pós-graduaÇÃo em Matemática

Aderson Araujo Silva Porto

Estimativas de altura e representação para superfícies de curvatura Gaussiana constante em $\mathbb{S}^{2} \times \mathbb{R} e \mathbb{H}^{2} \times \mathbb{R}$ 
Aderson Araujo Silva Porto

Estimativas de altura e representação para superfícies de curvatura Gaussiana constante em $\mathbb{S}^{2} \times \mathbb{R} e \mathbb{H}^{2} \times \mathbb{R}$

Dissertação apresentada ao Curso de Matemática da UnB, como requisito para a obtenção parcial do grau de MESTRE em Matemática.

Orientador: João Paulo dos Santos Doutor em Matemática - UnB

Brasília 
Ficha catalográfica elaborada automaticamente, com os dados fornecidos pelo(a) autor(a)

Porto, Aderson Araujo Silva

Estimativas de altura e representação para superfícies de curvatura Gaussiana constante em $\mathrm{S}^{2} \mathrm{x}$ $\mathrm{R}$ e $\mathrm{H}^{2}$ x R / Aderson Araujo Silva Porto; orientador João Paulo dos Santos. -- Brasilia, 2015. $82 \mathrm{p}$.

Dissertação (Mestrado - Mestrado em Matemática) -Universidade de Brasília, 2015.

1. Superfícies com curvatura constante. 2. Espaços produto. 3. Estimativas de altura. 4. Fórmulas de representação. I. Santos, João Paulo dos, orient. II. Título. 


\section{Estimativas de altura e representação para superfícies de curvatura Gaussiana constante em $\mathbb{S}^{2} \times \mathbb{R} e \mathbb{H}^{2} \times \mathbb{R}$}

Dissertação apresentada ao Curso de Matemática da UnB, como requisito para a obtenção parcial do grau de MESTRE em Matemática.

Aprovado em 24 de abril de 2015

\section{BANCA EXAMINADORA}
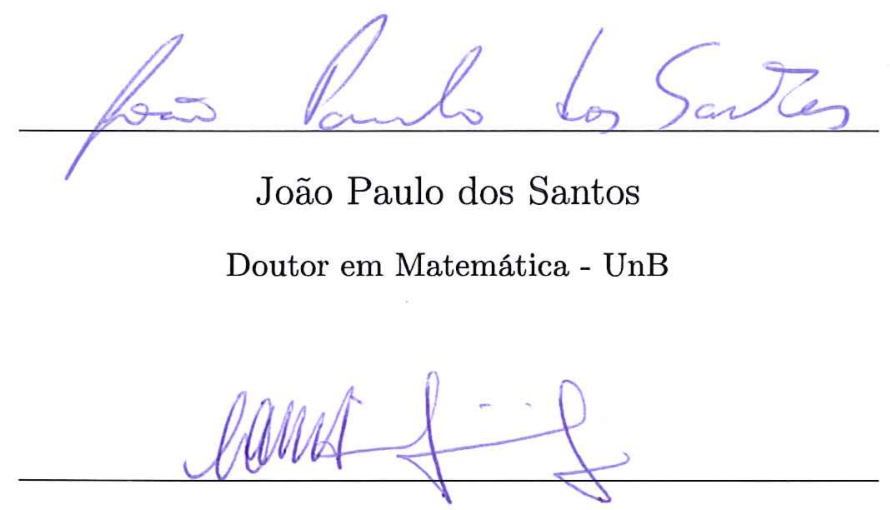

Luciana Maria Dias de Ávila Rodrigues

Doutor em Matemática - UnB

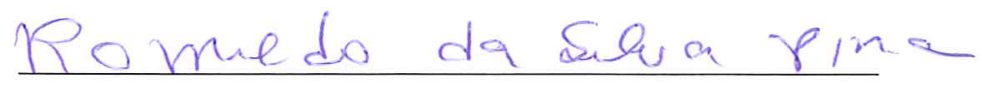

Romildo da Silva Pina

Doutor em Matemática - UFG 
Dedico este trabalho à minha família, especialmente à minha mãe. 


\section{Resumo}

Nesta dissertação, baseada em um artigo de Juan A. Aledo, José M. Espinar e José A. Gálvez, apresentamos estimativas de altura ótimas para superfícies em $\mathbb{S}^{2} \times \mathbb{R}$ e $\mathbb{H}^{2} \times \mathbb{R}$ com curvatura Gaussiana $K(I)$ constante e curvatura extrínseca positiva, caracterizando os casos extremos como superfícies de revolução. Além disso, apresentamos uma fórmula de representação para superfícies com curvatura Gaussiana constante em tais espaços ambientes, dando especial atenção aos casos de $K(I)=1$ em $\mathbb{S}^{2} \times \mathbb{R}$ e $K(I)=-1$ em $\mathbb{H}^{2} \times \mathbb{R}$.

Palavras-chaves: superfícies com curvatura constante, espaços produto, estimativas de altura, fórmula de representação. 


\begin{abstract}
In this master thesis, based on a paper of Juan A. Aledo, José M. Espinar and José A. Gálvez, we present optimal height estimates for surfaces in $\mathbb{S}^{2} \times \mathbb{R}$ and $\mathbb{H}^{2} \times \mathbb{R}$ with constant Gaussian curvature $K(I)$ and positive extrinsic curvature, characterizing the extreme cases as the revolution ones. Moreover, we present a representation formula for surfaces with constant Gaussian curvature in such ambient spaces, with special attention to the cases of $K(I)=1$ in $\mathbb{S}^{2} \times \mathbb{R}$ and $K(I)=-1$ in $\mathbb{H}^{2} \times \mathbb{R}$.

Keywords: surfaces with constant curvature, product spaces, height estimates, representation formula.
\end{abstract}




\section{Agradecimentos}

Acima de tudo, agradeço a Deus, fonte da minha força e conhecimento.

Agradeço a minha família, especialmente a minha mãe. O seu apoio e os sacrifícios que você fez para que eu tivesse a melhor educação me permitiram chegar até aqui.

Agradeço aos professores do meu curso de graduação em matemática, especialmente aos professores Marcus Vinícius, Mauro Rabelo, José Alfredo (in memoriam) e Noraí Rocco.

Agradeço aos professores do meu curso de mestrado: Ricardo Ruviaro, Noraí Rocco, Ary Vasconcelos e João Paulo.

Agradeço aos meus amigos e colegas do mestrado, em especial a Ilton, Elaine e Leandro, pelos nossos momentos de estudo; ao Valter por muitas vezes ter tirado as minhas dúvidas de geometria Riemanniana.

Agradeço a todos os funcionários do Departamento de Matemática, especialmente à Bruna Ribeiro de Vasconcelos, modelo de funcionária competente e dedicada. Agradeço pela presteza e profissionalismo com que você resolve os nossos problemas burocráticos.

Agradeço ao meu orientador, o professor João Paulo dos Santos, por quem tenho grande admiração e respeito. Sou grato por tudo que você me ensinou nos cursos de Geometria Diferencial e Geometria Riemanniana, pela paciência que você sempre teve comigo nas orientações. Seus conselhos tornaram possível este trabalho. Devo à você a quase totalidade dos meus conhecimentos sobre Geometria Diferencial.

Agradeço aos professores João Paulo dos Santos, Luciana Maria Dias de Ávila Rodrigues e Romildo da Silva Pina, que compuseram a banca avaliadora. A atenção e sugestões que deram me permitiram tornar este trabalho melhor.

Agradeço à CAPES e ao CNPq pelo apoio financeiro. 
"O temor do Senhor é o princípio do conhecimento; mas os insensatos desprezam a sabedoria e a instrução".

Provérbios 1:7 


\section{Sumário}

Introdução 1

1 Preliminares 3

1.1 Pares fundamentais . . . . . . . . . . . . . . . . . . . . 3

1.1.1 Complexificação de uma forma quadrática . . . . . . . . . . . . 9

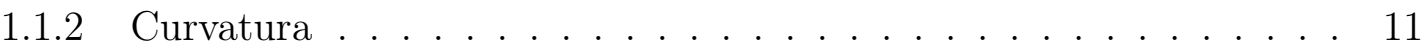

1.2 O tensor de Codazzi . . . . . . . . . . . . . . . . . . 12

1.3 Parametrizações especiais . . . . . . . . . . . . . . . 16

1.3.1 Parâmetros isotérmicos . . . . . . . . . . . . . . 16

1.3.2 Parâmetros duplamente ortogonais . . . . . . . . . . . . . . . . . 19

1.3.3 Parâmetros assintóticos . . . . . . . . . . . . . . . . . . 21

1.4 Formas Espaciais . . . . . . . . . . . . . . . . . . . 22

1.4.1 O espaço Euclidiano . . . . . . . . . . . . . . . 23

1.4.2 A esfera Euclidiana . . . . . . . . . . . . . . . . 23

1.4.3 O espaço hiperbólico . . . . . . . . . . . . . . . . . 23

1.5 Equações fundamentais . . . . . . . . . . . . . . . . . . . 24

1.5.1 Segunda forma fundamental . . . . . . . . . . . . . . . 25

1.5.2 As equações fundamentais de uma imersão isométrica . . . . . . . . 26

1.6 O princípio do Máximo . . . . . . . . . . . . . . . . . . . . . . . 31

2 Espaços produto $\quad 34$

2.1 Aspectos gerais . . . . . . . . . . . . . . . . . 34

2.2 Equações de compatibilidade . . . . . . . . . . . . . . . . . . . . 37 
3.1 Estimativas de altura para superfícies de curvatura Gaussiana constante . . 49

4 Representação

4.1 Representação de $K(I)$-superfícies com $K>0 \ldots \ldots$. . . . . . 63

4.2 Representação de $K(I)$-superfícies com $K<0$. . . . . . . . . . . . . 70

$\begin{array}{lll}5 & \text { Resumo e aplicações } & 74\end{array}$

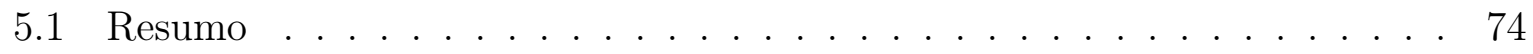

5.2 Aplicações . . . . . . . . . . . . . . . . . . . . 80

$\begin{array}{ll}\text { Referências Bibliográficas } & 83\end{array}$ 


\section{Introdução}

Neste trabalho vamos lidar com superfícies de curvatura Gaussiana constante nos espaços ambientes $\mathbb{S}^{2} \times \mathbb{R}$ e $\mathbb{H}^{2} \times \mathbb{R}$. Estes dois espaços ambientes são chamados de espaços produto, que são variedades tridimensionais dadas pelo produto de uma superfície Riemanniana $\left(\mathbb{S}^{2}\right.$ ou $\left.\mathbb{H}^{2}\right)$ com a reta real $\mathbb{R}$. Estes espaços estão sendo muito estudados. Citamos, como exemplos, [2] onde são estudadas superfícies completas; em [4] são dadas condições necessárias e suficientes para que uma variedade Riemanniana $n$-dimensional seja isometricamente imersa nos espaços produto mais gerais $\mathbb{S}^{n} \times \mathbb{R}$ e $\mathbb{H}^{n} \times \mathbb{R}$.

$\mathrm{Na}$ maior parte deste trabalho, não precisaremos distinguir explicitamente entre $\mathbb{S}^{2} \times \mathbb{R}$ e $\mathbb{H}^{2} \times \mathbb{R}$, assim usaremos a notação usual $\mathbb{M}^{2}(\varepsilon) \times \mathbb{R}$, com $\varepsilon=1,-1$, onde $\mathbb{M}^{2}(1)=\mathbb{S}^{2}$ e $\mathbb{M}^{2}(-1)=\mathbb{H}^{2}$. Vamos considerar $\mathbb{S}^{2} \times \mathbb{R}$ como uma hipersuperfície do espaço Euclidiano usual $\mathbb{R}^{4}$ e $\mathbb{H}^{2} \times \mathbb{R}$ como uma subvariedade Riemanniana do espaço de Lorentz $\mathbb{L}^{4}$. Sejam $\Sigma$ uma superfície Riemanniana e $\psi: \Sigma \rightarrow \mathbb{M}^{2}(\varepsilon) \times \mathbb{R}$ uma imersão. Chamaremos de função altura $h$ a quarta coordenada de tal imersão, e de função ângulo $\nu$ a quarta coordenada do campo normal unitário $N$.

A referência principal que utilizamos é o artigo [1]. Os dois temas principais deste artigo são estimativas de altura e representação para superfícies de curvatura Gaussiana constante. A estimativa de altura estabelece uma cota máxima ótima para a altura medida sobre um conjunto $\Omega \subset \mathbb{M}^{2}(\varepsilon)$. Veremos também que tal cota é atingida se, e somente se, $\psi$ é um hemisfério de uma superfície completa de rotação. Lidaremos somente com superfícies de curvatura Gaussiana constante, mas resultados semelhantes foram encontrados pelos mesmos autores para superfícies de curvatura média positiva constante no artigo [3].

O teorema de representação nos permite escrever, para superfícies transversas, os coeficientes da primeira e segunda formas fundamentais em termos da função altura $h, \nu$ e da curvatura Gaussiana $K(I)$, suposta constante. Reciprocamente, se $K(I)$ é constante e as funções $h$ e $\nu$ satisfazem algumas condições, então existe uma única imersão, a menos de isometria do espaço ambiente, cujos coeficientes de sua primeira e segunda forma fundamentais são dados em termos de $h, \nu$ e $K(I)$. Assim, teoremas de representação 
são importantes ferramentas, pois permitem garantir a existência de imersões e também conhecer as expressões para suas primeira e segunda formas fundamentais. Como exemplo de utilização de representação, citamos [8], no qual os autores utilizaram teoremas deste tipo para classificar superfícies helicoidais planas no espaço hiperbólico $\mathbb{H}^{3}$.

A presente dissertação está dividida em cinco capítulos da seguinte forma:

O capítulo 1 é reservado para revisão de alguns conceitos básicos de geometria Riemanniana, fixar a notação utilizada ao longo do texto, introdução de algumas parametrizações especiais, dedução das equações fundamentais de uma imersão isométrica (equações de Gauss, Ricci e Codazzi) e também para recordar o Princípio do Máximo de Hopf.

No capítulo 2 , discorremos sobre os espaços produto $\mathbb{S}^{2} \times \mathbb{R}$ e $\mathbb{H}^{2} \times \mathbb{R}$. Aqui damos a definição, vemos algumas propriedades e deduzimos as equações de compatibilidade em tais espaços.

O capítulo 3 iniciamos com a introdução de uma forma quadrática. Damos dois exemplos de superfícies completas. No primeiro teorema deste capítulo vemos que se tal forma quadrática é identicamente nula, então a superfície deve ser uma parte de uma superfície de revolução. E o segundo teorema deste capítulo estabelece a estimativa de altura.

No capítulo 4, dividido em duas seções, temos o teorema da representação. Na primeira seção são consideradas superfícies com curvatura extrínseca positiva, e na segunda seção, superfícies com curvatura extrínseca negativa. Damos especial atenção ao caso em que $K(I)=1$ em $\mathbb{S}^{2} \times \mathbb{R}$ e $K(I)=-1$ em $\mathbb{H}^{2} \times \mathbb{R}$. Nestes casos, uma das condições de integrabilidade torna-se a clássica equação sinh-Gordon elíptica e a sinh-Gordon hiperbólica, respectivamente.

No capítulo 5, em sua primeira seção, fizemos um resumo dos principais resultados apresentados nesta dissertação. Na segunda seção, apresentamos alguns resultados em cuja demonstração são utilizados estimativas de altura e representação semelhantes aos que foram estudados neste trabalho. Fizemos isto com o intuito de demonstrar a relevância dos temas que aqui foram abordados.

Ao longo deste trabalho, iremos supor que a diferenciabilidade a que nos referimos é sempre $C^{\infty}$. 


\section{Preliminares}

Neste capítulo, vamos introduzir alguns conceitos básicos, fixar a notação utilizada, enunciar alguns resultados, bem como apresentar algumas equações fundamentais para uma imersão isométrica que serão utilizadas em todo o trabalho.

\subsection{Pares fundamentais}

Neste trabalho, usaremos o termo superfície para nos referir a uma variedade diferenciável orientável de dimensão 2. Dada uma superfície $\Sigma$, denotaremos por

- $\mathcal{X}(\Sigma)$ o conjunto de campos de vetores diferenciáveis em $\Sigma$ e por $C^{\infty}(\Sigma)$ o conjunto das funções reais diferenciáveis em $\Sigma$.

- $[$, ] o colchete de Lie de campos.

- $\Lambda^{r}(\Sigma)$ o conjunto de aplicações diferenciáveis de produto cartesiano de $\mathcal{X}(\Sigma) r$ vezes em $\mathcal{X}(\Sigma)$, isto é

$$
T: \mathcal{X}(\Sigma) \times \cdots \times \mathcal{X}(\Sigma) \rightarrow \mathcal{X}(\Sigma)
$$

- $\tau^{r}(\Sigma)$ o subconjunto de aplicações de $\Lambda^{r}$ que são $C^{\infty}(\Sigma)$ - multilineares.

- $\mathcal{Q}(\Sigma)$ o conjunto de formas bilineares simétricas sobre $\Sigma$, que identificaremos com suas formas quadráticas associadas. $\mathcal{M}(\Sigma) \subset \mathcal{Q}(\Sigma)$ ao subconjunto formada por aquelas que definem uma métrica Riemanniana $\mathcal{R}(\Sigma)$ ou Lorentziana $\mathcal{L}(\Sigma)$ sobre $\Sigma$, isto é, $\mathcal{M}(\Sigma) \equiv \mathcal{R}(\Sigma) \cup \mathcal{L}(\Sigma)$.

Uma forma bilinear em $\Sigma$ é uma aplicação

$$
T: \mathcal{X}(\Sigma) \times \mathcal{X}(\Sigma) \rightarrow C^{\infty}(\Sigma)
$$

tal que

(i) $T(f X, Y)=f T(X, Y)=T(X, f Y)$ para quaisquer $X, Y \in \mathcal{X}(\Sigma), f \in C^{\infty}(\Sigma)$; 
(ii) $T(X+Y, Z)=T(X, Z)+T(Y, Z)$ e $T(X, Y+Z)=T(X, Y)+T(X, Z)$ para quaisquer $X, Y, Z \in \mathcal{X}(\Sigma)$.

A propriedade (i) implica que o valor de $T(X, Y)$ em $p \in \Sigma$ depende apenas dos valores de $X$ e $Y$ em $p$. Assim, para cada $p \in \Sigma$, a forma bilinear $T$ define uma aplicação bilinear

$$
T_{p}: T_{p} \Sigma \times T_{p} \Sigma \rightarrow \mathbb{R}
$$

em que $T_{p} \Sigma$ denota o plano tangente a $\Sigma$ em $p$.

Diz-se que a forma bilinear $T$ é simétrica se $T(X, Y)=T(Y, X)$ para quaisquer $X, Y \in \mathcal{X}(\Sigma)$. Equivalentemente, $T$ é simétrica se $T_{p}$ é uma aplicação bilinear simétrica para todo $p \in \Sigma$. Se, além disso, para cada $p \in \Sigma$ a aplicação bilinear $T_{p}$ é positiva definida, isto é, $T_{p}\left(X_{p}, X_{p}\right)>0$ para qualquer $X_{p} \in T_{p} \Sigma$, então $T$ é uma métrica Riemanniana em $\Sigma$.

Se $T$ é uma forma bilinear simétrica em $\Sigma$, sua forma quadrática associada é a aplicação

$$
\hat{T}: \mathcal{X}(\Sigma) \rightarrow C^{\infty}(\Sigma)
$$

dada por $\hat{T}(X)=T(X, X)$ para qualquer $X \in \mathcal{X}(\Sigma)$. Em geral, usamos o mesmo símbolo para representar uma forma bilinear simétrica e sua forma quadrática associada.

Vejamos como se exprime uma forma bilinear simétrica em uma superfície $\Sigma$ em termos de uma sistema de coordenadas $(x, U)$ em um aberto $U \subset \mathbb{R}^{2}$, com $x=\left(x_{1}, x_{2}\right)$. Denotamos por $\frac{\partial}{\partial x_{1}}, \frac{\partial}{\partial x_{2}}$ os campos coordenados e por $d x_{1}, d x_{2}$ as 1-formas duais, ou seja

$$
d x_{i}\left(\frac{\partial}{\partial x_{j}}\right)= \begin{cases}1, & \text { se } i=j \\ 0, & \text { se } i \neq j\end{cases}
$$

Então, para cada $p \in x(U)$, as aplicações bilineares $\left\{d x_{i} \otimes d x_{j} \mid i, j=1,2\right\}$ formam uma base do espaço de aplicações bilineares $\left\{\alpha: T_{p} \Sigma \times T_{p} \Sigma \rightarrow \mathbb{R}\right\}$. Portanto, toda forma bilinear $T$ em $\Sigma$ se exprime em $x(U)$ por

$$
T=\sum_{i, j=1}^{2} T_{i j} d x_{i} \otimes d x_{j}
$$


em que $T_{i j}=T\left(\frac{\partial}{\partial x_{i}}, \frac{\partial}{\partial x_{j}}\right), 1 \leq i, j \leq 2$, são funções reais diferenciáveis em $x(U)$. Em particular se $T$ é simétrica, sua forma quadrática associada (denotada com o mesmo símbolo $T)$ se exprime em $x(U)$ por

$$
T=E d x_{1}^{2}+2 F d x_{1} d x_{2}+G d x_{2}^{2}
$$

em que $E=T_{11}, F=T_{12}=T_{21}$ e $G=T_{22}$.

Definição 1.1. A um par

$$
(I, I I) \in \mathcal{R}(\Sigma) \times \mathcal{Q} \equiv \mathcal{P}(\Sigma)
$$

chamaremos par fundamental sobre $\Sigma$. Ao conjunto $\mathcal{P}(\Sigma)$ chamaremos conjunto de pares fundamentais.

Ao par fundamental $(I, I I)$ está associado $S: \mathcal{X}(\Sigma) \rightarrow \mathcal{X}(\Sigma)$ chamado operador forma de $(I, I I)$, dado por

$$
I I(X, Y)=I(S X, Y)
$$

Reciprocamente, dada uma forma quadrática $I I$ sobre a superfície, podemos definir $S$ associada a $I I$ como o único endomorfismo $S$ que satisfaz

$$
I I(X, Y)=I(S X, Y)
$$

Escrevendo

$$
\begin{gathered}
I=E d x^{2}+2 F d x d y+G d y^{2}, \\
I I=e d x^{2}+2 f d x d y+g d y^{2},
\end{gathered}
$$

definimos

$$
\begin{aligned}
& H=H(I, I I)=\frac{1}{2} \frac{E g-2 f F+G e}{E G-F^{2}}, \\
& K=K(I, I I)=\frac{e g-f^{2}}{E G-F^{2}}
\end{aligned}
$$

as curvaturas média e extrínseca, respectivamente, do par $(I, I I)$.

A partir da definição anterior, definimos as curvaturas principais do par como sendo

$$
\begin{aligned}
& k_{1}=H+\sqrt{H^{2}-K}, \\
& k_{2}=H-\sqrt{H^{2}-K} .
\end{aligned}
$$


De onde observamos que denotamos $k_{1}$ como a curvatura principal maior. Além disso, denotaremos por a a curvatura assimétrica, isto é,

$$
a=a(I, I I)=H^{2}-K^{2}=\frac{\left(k_{1}-k_{2}\right)^{2}}{4} .
$$

Diremos que o par $(I, I I)$ é umbílico em $p \in \Sigma$ se $I I$ é proporcional a $I$, isto é $I I(p)=\lambda(p) I(p)$, ou equivalentemente, as curvaturas principais coincidem nesse ponto. No que se segue denotaremos por $\mathcal{U}_{(I, I I)}$ o conjunto de pontos umbílicos de $\Sigma$ associados a $(I, I I)$ e se não houver confusão somente escreveremos $\mathcal{U}$. Claramente tal conjunto é um fechado da superfície e ainda $\Sigma^{\prime}=\left(\Sigma \backslash \mathcal{U}_{(I, I I)}\right) \cup \operatorname{int}\left(\mathcal{U}_{(I, I I)}\right)$ é um conjunto denso em $\Sigma$.

Sabe-se que todo endomorfismo autoadjunto $S$ é diagonalizável quando $I$ é Riemanniana, isto é, dado um ponto $p \in \Sigma$, existe uma base ortonormal do espaço tangente $\left\{e_{1}, e_{2}\right\} \in T_{p} S$ tal que

$$
S\left(e_{i}\right)=k_{i}(p) e_{i}, \quad i=1,2
$$

isto é, os autovalores, $k_{i}$ de $S$ são as curvaturas principais do par. Logo, a curvatura média (respectivamente curvatura extrínseca) do par é metade do traço (respectivamente determinante) do endomorfismo $S$.

Denotaremos por $\left\{\frac{\partial}{\partial x}, \frac{\partial}{\partial y}\right\}$ os campos coordenados associados a parametrização e por $\nabla$ a conexão de Levi-Civita de $I$. Então as funções $\Gamma_{i j}^{k}$ definidas em $U$ por

$$
\begin{aligned}
& \nabla_{\frac{\partial}{\partial x}} \frac{\partial}{\partial x}=\Gamma_{11}^{1} \frac{\partial}{\partial x}+\Gamma_{11}^{2} \frac{\partial}{\partial y} \\
& \nabla_{\frac{\partial}{\partial x}} \frac{\partial}{\partial y}=\Gamma_{12}^{1} \frac{\partial}{\partial x}+\Gamma_{12}^{2} \frac{\partial}{\partial y} \\
& \nabla_{\frac{\partial}{\partial y}} \frac{\partial}{\partial y}=\Gamma_{22}^{1} \frac{\partial}{\partial x}+\Gamma_{22}^{2} \frac{\partial}{\partial y}
\end{aligned}
$$


são os coeficientes da conexão $\nabla$ em $U$ associados aos parâmetros $(x, y)$, chamados símbolos de Christoffel associados a métrica $I$, e veem dados por

$$
\begin{aligned}
\Gamma_{11}^{1} & =\frac{1}{2\left(E G-F^{2}\right)}\left(G E_{x}-2 F F_{x}+F E_{y}\right), \\
\Gamma_{11}^{2} & =-\frac{1}{2\left(E G-F^{2}\right)}\left(E E_{y}-2 E F_{x}+F E_{x}\right), \\
\Gamma_{12}^{1} & =\frac{1}{2\left(E G-F^{2}\right)}\left(G E_{y}-F G_{x}\right), \\
\Gamma_{12}^{2} & =\frac{1}{2\left(E G-F^{2}\right)}\left(E G_{x}-F E_{y}\right), \\
\Gamma_{22}^{1} & =-\frac{1}{2\left(E G-F^{2}\right)}\left(G G_{x}-2 G F_{y}+F G_{y}\right), \\
\Gamma_{22}^{2} & =\frac{1}{2\left(E G-F^{2}\right)}\left(E G_{y}-2 F F_{y}+F G_{x}\right)
\end{aligned}
$$

que podem ser calculados facilmente a partir de (1.2).

Seja $f: \Sigma \rightarrow \mathbb{R}$ uma aplicação diferenciável. O gradiente da aplicação $f$ é uma aplicação diferenciável grad $f: \Sigma \rightarrow \mathcal{X}(\Sigma)$ que associa a cada ponto $p \in \Sigma$ um vetor $\operatorname{grad} f(p) \in T_{p} \Sigma$ tal que

$$
d f_{p}(v)=I(\operatorname{grad} f(p), v), \quad \forall v \in T_{p} \Sigma
$$

Se

$$
I=E d u^{2}+2 F d u d v+G d v^{2}
$$

pode-se mostrar que

$$
\operatorname{grad} f=\frac{G f_{u}-F f_{v}}{E G-F^{2}} \partial_{u}+\frac{E f_{v}-F f_{u}}{E G-F^{2}} \partial_{v}
$$

(veja [7], Exercício 14, pág. 120).

Seja $u:(M, g) \rightarrow(N, h)$ uma aplicação diferenciável entre as variedades Riemannianas $M$ e $N$, de dimensões $m$ e $n$, respectivamente. Sejam $\left(x^{i}\right)$ e $\left(y^{\alpha}\right)$ sistemas de coordenadas locais em $M$ e $N$, respectivamente. Com essas coordenadas locais, expressamos a aplicação $u$ por

$$
u(x)=\left(u^{1}\left(x^{1}, \ldots, x^{m}\right), \ldots, u^{n}\left(x^{1}, \ldots, x^{m}\right)\right)=\left(u^{\alpha}\left(x^{i}\right)\right) .
$$

A aplicação $u$ é uma aplicação harmônica se $u$ satisfaz o seguinte sistema de equações

$$
\Delta u^{\alpha}+\sum_{i, j=1}^{m} \sum_{\beta, \gamma=1}^{n} g^{i j} \Gamma_{\beta \gamma}^{\prime \alpha}(u) \frac{\partial u^{\beta}}{\partial x^{i}} \frac{\partial u^{\gamma}}{\partial x^{j}}=0, \quad 1 \leq \alpha \leq n,
$$


onde

$$
\Delta u^{\alpha}=\sum_{i, j=1}^{m} g^{i j}\left\{\frac{\partial^{2} u^{\alpha}}{\partial x^{i} \partial x^{j}}-\sum_{k=1}^{m} \Gamma_{i j}^{k} \frac{\partial u^{\alpha}}{\partial x^{k}}\right\}
$$

é o Laplaciano de $u$ em $M, g^{i j}$ é a matriz inversa de $g_{i j}$, e $\Gamma_{i j}^{k}$ e $\Gamma_{\beta \gamma}^{\prime a}$ representam os coeficiente da conexão de Levi-Civita em $M$ e $N$, respectivamente.

Agora, considere em

$$
\mathbb{H}^{n}=\left\{\left(x_{1}, \ldots, x_{n}\right) \in \mathbb{R}^{n} ; x_{n}>0\right\}
$$

a métrica

$$
g_{i j}=\frac{\delta_{i j}}{F^{2}}
$$

onde $F$ é uma função positiva diferenciável. Escreveremos $g^{i j}=F^{2} g_{i j}$ para indicar a matriz inversa de $g_{i j}$, e façamos $\ln F=f$. Indicaremos $\frac{\partial}{\partial x_{j}} f=f_{j}$. Então podemos concluir que (veja cap. 8 de [5]):

$$
\Gamma_{i j}^{k}=0, \quad \Gamma_{i j}^{i}=-f_{j}, \quad \Gamma_{i i}^{j}=f_{j}, \quad \Gamma_{i j}^{j}, \quad \Gamma_{i i}^{i}=-f_{i} .
$$

Sejam $(M, g)$ e $(N, h)$ 2-variedades Riemannianas orientáveis. Sejam $\left(x^{1}, x^{2}\right)$ e $\left(y^{1}, y^{2}\right)$ coordenadas isotérmicas em $M$ e $N$, respectivamente, por meio das quais, $g$ e $h$ são expressas localmente como

$$
\begin{aligned}
& g=\sigma(w)^{2}\left(\left(d x^{1}\right)^{2}+\left(d x^{2}\right)^{2}\right)=\sigma^{2} d w d \bar{w}, \quad \sigma>0, \\
& h=\rho(v)^{2}\left(\left(d y^{1}\right)^{2}+\left(d y^{2}\right)^{2}\right)=\rho^{2} d v d \bar{v}, \quad \rho>0,
\end{aligned}
$$

onde $w=x^{1}+i x^{2}$ e $v=y^{1}+i y^{2}$. Nessas condições, devido à (1.13), o sistema (1.12) assume a forma seguinte

$$
\sigma^{-2} \sum_{i=1}^{2}\left[\frac{\partial^{2} u^{\alpha}}{\left(\partial x^{i}\right)^{2}}+\sum_{\beta, \gamma=1}^{2} \Gamma_{\beta \gamma}^{\prime \alpha}(u) \frac{\partial u^{\beta}}{\partial x^{i}} \frac{\partial u^{\gamma}}{\partial x^{i}}\right]=0, \quad \text { para } \alpha=1,2 .
$$

Este sistema é equivalente à equação

$$
\sigma^{-1}\left(\frac{\partial^{2} u}{\partial w \partial \bar{w}}+\frac{2}{\rho} \frac{\partial \rho}{\partial v} \frac{\partial u}{\partial w} \frac{\partial u}{\partial \bar{w}}\right)=0
$$

onde

$$
\frac{\partial u}{\partial w}=\frac{1}{2}\left(\frac{\partial u}{\partial x^{1}}-i \frac{\partial u}{\partial x^{2}}\right) \quad \text { e } \quad \frac{\partial u}{\partial \bar{w}}=\frac{1}{2}\left(\frac{\partial u}{\partial x^{1}}+i \frac{\partial u}{\partial x^{2}}\right)
$$




\subsubsection{Complexificação de uma forma quadrática}

Uma ferramenta que usaremos com grande frequência é a análise complexa. Dito isto, recordemos como introduzir a variável complexa em uma superfície. Chamaremos fibrado tangente complexificado de $T \Sigma$ o fibrado

$$
T^{\mathbb{C}} \Sigma=\{X+i Y: X, Y \in \mathcal{X}(S)\}
$$

Pode-se provar que se $\{X, Y\}$ é uma base de $T \Sigma$, então $\{X, i X, Y, i Y\}$ é base de $T^{\mathbb{C}} \Sigma$ como fibrado real, e $\{X+i Y, X-i Y\}$ é base como fibrado complexo. Em particular, dada uma parametrização $(x, y)$ em um aberto $U$ de $\Sigma$, se considerarmos o parâmetro complexo $z=x+i y$, podemos definir os campos coordenados

$$
\begin{aligned}
& \frac{\partial}{\partial z}=\frac{1}{2}\left(\frac{\partial}{\partial x}-i \frac{\partial}{\partial y}\right), \\
& \frac{\partial}{\partial \bar{z}}=\frac{1}{2}\left(\frac{\partial}{\partial x}+i \frac{\partial}{\partial y}\right),
\end{aligned}
$$

de modo que $\left\{\frac{\partial}{\partial z}, \frac{\partial}{\partial \bar{z}}\right\}$ é uma base local para $T^{\mathbb{C}} \Sigma$.

Além disso, podemos definir as 1-formas complexas locais

$$
\begin{aligned}
& d z=d x+i d y, \\
& d \bar{z}=d x-i d y,
\end{aligned}
$$

que formam a base dual de $\left\{\frac{\partial}{\partial z}, \frac{\partial}{\partial \bar{z}}\right\}$. Assim, trabalharemos indistintamente com uma parametrização $(x, y)$ ou um parâmetro complexo $z=x+i y$. Portanto, podemos reescrever

$$
\begin{gathered}
I=p d z^{2}+2 \lambda|d z|^{2}+\bar{p} d \bar{z}^{2}, \\
I I=q d z^{2}+2 \rho|d z|^{2}+\bar{q} d \bar{z}^{2} .
\end{gathered}
$$

De (1.2) e (1.3), concluímos que

$$
\begin{aligned}
p & =\frac{1}{4}(E-G-2 i F), \\
\lambda & =\frac{1}{2}(E+G), \\
q & =\frac{1}{4}(e-g-2 i f), \\
\rho & =\frac{1}{2}(e+g) .
\end{aligned}
$$


Podemos assim comprovar com um cálculo direto de (1.4) e (1.5) que

$$
\begin{aligned}
& H=H(I, I I)=\frac{p \bar{q}-2 \lambda \rho+\bar{p} q}{2\left(|p|^{2}-\lambda^{2}\right)}, \\
& K=K(I, I I)=\frac{|q|^{2}-\rho^{2}}{|p|^{2}-\lambda^{2}} .
\end{aligned}
$$

Podemos, ainda, expressar os coeficientes da conexão $\nabla$ associados a $z$ e $\bar{z}$ como

$$
\begin{aligned}
& \nabla_{\frac{\partial}{\partial z} \frac{\partial}{\partial z}}={ }^{\mathbb{C}} \Gamma_{11}^{1} \frac{\partial}{\partial z}+{ }^{\mathbb{C}} \Gamma_{11}^{2} \frac{\partial}{\partial \bar{z}} \\
& \nabla_{\frac{\partial}{\partial z}} \frac{\partial}{\partial \bar{z}}={ }^{\mathbb{C}} \Gamma_{12}^{1} \frac{\partial}{\partial z}+{ }^{\mathbb{C}} \Gamma_{12}^{2} \frac{\partial}{\partial \bar{z}} \\
& \nabla_{\frac{\partial}{\partial \bar{z}} \frac{\partial}{\partial \bar{z}}}={ }^{\mathbb{C}} \Gamma_{22}^{1} \frac{\partial}{\partial z}+{ }^{\mathbb{C}} \Gamma_{22}^{2} \frac{\partial}{\partial \bar{z}}
\end{aligned}
$$

sendo ${ }^{\mathbb{C}} \Gamma_{i j}^{k}$ os símbolos de Christoffel da conexão associados a parametrização $(z, \bar{z})$.

Vejamos como se relacionam os símbolos de Christoffel $\Gamma_{i j}^{k}$ associados a parametrização $(x, y)$ com os símbolos ${ }^{\mathbb{C}} \Gamma_{i j}^{k}$ associados a parametrização $(z, \bar{z})$, onde $z=x+i y$ :

$$
\begin{aligned}
\nabla_{\frac{\partial}{\partial z}} \frac{\partial}{\partial z}= & \frac{1}{4} \nabla_{\frac{\partial}{\partial x}-i \frac{\partial}{\partial y}}\left(\frac{\partial}{\partial x}-i \frac{\partial}{\partial y}\right) \\
= & \frac{1}{4}\left(\nabla_{\frac{\partial}{\partial x}} \frac{\partial}{\partial x}-2 i \nabla_{\frac{\partial}{\partial x}} \frac{\partial}{\partial y}-\nabla_{\frac{\partial}{\partial y}} \frac{\partial}{\partial y}\right) \\
= & \frac{1}{4}\left(\left(\Gamma_{11}^{1}-2 i \Gamma_{12}^{1}-\Gamma_{22}^{1}\right) \frac{\partial}{\partial x}+\left(\Gamma_{11}^{2}-2 i \Gamma_{12}^{2}-\Gamma_{22}^{2}\right) \frac{\partial}{\partial y}\right) \\
= & \frac{1}{4}\left(\Gamma_{11}^{1}-\Gamma_{22}^{1}+2 \Gamma_{12}^{2}+i\left(\Gamma_{11}^{2}-\Gamma_{22}^{2}-2 \Gamma_{12}^{1}\right)\right) \frac{\partial}{\partial z} \\
& +\frac{1}{4}\left(\Gamma_{11}^{1}-\Gamma_{22}^{1}-2 \Gamma_{12}^{2}-i\left(\Gamma_{11}^{2}-\Gamma_{22}^{2}+2 \Gamma_{12}^{1}\right)\right) \frac{\partial}{\partial \bar{z}} .
\end{aligned}
$$

De forma análoga podemos relacionar os outros, obtendo

$$
\begin{aligned}
& { }^{\mathbb{C}} \Gamma_{11}^{1}=\frac{1}{4}\left(\Gamma_{11}^{1}-\Gamma_{22}^{1}+2 \Gamma_{12}^{2}+i\left(\Gamma_{11}^{2}-\Gamma_{22}^{2}-2 \Gamma_{12}^{1}\right)\right), \\
& { }^{\mathbb{C}} \Gamma_{11}^{2}=\frac{1}{4}\left(\Gamma_{11}^{1}-\Gamma_{22}^{1}-2 \Gamma_{12}^{2}-i\left(\Gamma_{11}^{2}-\Gamma_{22}^{2}+\Gamma_{12}^{1}\right)\right), \\
& { }^{\mathbb{C}} \Gamma_{12}^{1}=\frac{1}{4}\left(\Gamma_{11}^{1}+\Gamma_{22}^{1}+i\left(\Gamma_{11}^{2}+\Gamma_{22}^{2}\right)\right) .
\end{aligned}
$$

Prosseguindo, usando (1.24) e (1.25), podemos relacionar os outros coeficientes, obtendo as seguintes relações

$$
\begin{aligned}
& { }^{\mathbb{C}} \Gamma_{11}^{1}={ }^{\mathbb{C}} \overline{\Gamma_{22}^{2}}, \\
& { }^{\mathbb{C}} \Gamma_{11}^{2}={ }^{\mathbb{C}} \overline{\Gamma_{22}^{1}} \\
& { }^{\mathbb{C}} \Gamma_{12}^{1}={ }^{\mathbb{C}} \overline{\Gamma_{12}^{2}}
\end{aligned}
$$


Além disso, um cálculo direto, substituindo as equações (1.17) e (1.18), nas expressões (1.6) - (1.11), obtemos que

$$
\begin{aligned}
{ }^{\mathbb{C}} \Gamma_{11}^{1} & =\frac{1}{2\left(|p|^{2}-\lambda^{2}\right)}\left(\bar{p} p_{z}-2 \lambda \lambda_{z}+\lambda p_{\bar{z}}\right), \\
{ }^{\mathbb{C}} \Gamma_{11}^{2} & =-\frac{1}{2\left(|p|^{2}-\lambda^{2}\right)}\left(p p_{\bar{z}}-2 p \lambda_{z}+\lambda p_{z}\right), \\
{ }^{\mathbb{C}} \Gamma_{12}^{1} & =\frac{1}{2\left(|p|^{2}-\lambda^{2}\right)}\left(\bar{p} p_{\bar{z}}-\lambda \bar{p}_{z}\right), \\
{ }^{\mathbb{C}} \Gamma_{12}^{2} & =\frac{1}{2\left(|p|^{2}-\lambda^{2}\right)}\left(p \bar{p}_{z}-\lambda p_{\bar{z}}\right), \\
{ }^{C} & \Gamma_{22}^{1}=-\frac{1}{2\left(|p|^{2}-\lambda^{2}\right)}\left(\bar{p} \bar{p}_{z}-2 \bar{p} \lambda_{\bar{z}}+\lambda \bar{p}_{\bar{z}}\right), \\
{ }^{\mathbb{C}} \Gamma_{22}^{2} & =\frac{1}{2\left(|p|^{2}-\lambda^{2}\right)}\left(p \bar{p}_{\bar{z}}-2 \lambda \lambda_{\bar{z}}+\lambda \bar{p}_{z}\right) .
\end{aligned}
$$

O superíndice $\mathbb{C}$ se refere claramente a parametrização $(z, \bar{z})$, do qual prescindiremos sempre e quando não existir confusão.

\subsubsection{Curvatura}

Um elemento de muita importância associado com a conexão $\nabla$ é o tensor de curvatura $R$, que é uma aplicação trilinear dado por

$$
R(X, Y) Z=\nabla_{Y} \nabla_{X} Z-\nabla_{X} \nabla_{Y} Z+\nabla_{[X, Y]} Z, \quad X, Y, Z \in \mathcal{X}(\Sigma)
$$

que mede o quanto deixa a métrica de ser plana, isto é, tem curvatura zero (espaço Euclidiano). É um cálculo direto demonstrar que $R$ é linear em todas as suas variáveis e antisimétrica em suas duas primeiras variáveis.

Associada ao tensor de curvatura (1.38), está a curvatura seccional de $I$, que é dada por

$$
K(I)(p)=\frac{\left\langle R\left(X_{p}, Y_{p}\right) X_{p}, Y_{p}\right\rangle}{\left\|X_{p} \wedge Y_{p}\right\|}, \quad p \in S
$$

onde $X_{p}, Y_{p} \in T_{p} S$ são linearmente independentes e

$$
\left\|X_{p} \wedge Y_{p}\right\|=\sqrt{\left\|X_{p}\right\|^{2}\left\|Y_{p}\right\|^{2}-\left\langle X_{p}, Y_{p}\right\rangle^{2}}
$$

Notemos ainda que a definição de $K(I)$ não depende dos vetores escolhidos, mas exclusivamente do ponto onde estamos trabalhando. Além disso, um fato bastante 
conhecido é que $K(I)$ determina completamente a curvatura $R$, quando estamos em superfícies, mediante a identidade

$$
R(X, Y) Z=K(I)(\langle X, Z\rangle Y-\langle Y, Z\rangle X)
$$

Nos referiremos a $K(I)$ como a curvatura intrínseca ou curvatura de Gauss, quando estivermos trabalhando com superfícies.

Vamos escrever o tensor de curvatura e a curvatura intrínseca em um sistema de coordenadas. Assim, dada uma parametrização $(x, y)$, temos que

$$
\begin{aligned}
R\left(\frac{\partial}{\partial x}, \frac{\partial}{\partial y}\right) \frac{\partial}{\partial x}= & \nabla_{\frac{\partial}{\partial y}} \nabla_{\frac{\partial}{\partial x}} \frac{\partial}{\partial x}-\nabla_{\frac{\partial}{\partial x}} \frac{\partial}{\partial y} \frac{\partial}{\partial x} \\
= & \nabla_{\frac{\partial}{\partial y}}\left(\Gamma_{11}^{1} \frac{\partial}{\partial x}+\Gamma_{11}^{2} \frac{\partial}{\partial y}\right)-\nabla_{\frac{\partial}{\partial x}}\left(\Gamma_{12}^{1} \frac{\partial}{\partial x}+\Gamma_{12}^{2} \frac{\partial}{\partial y}\right) \\
= & \left(\left(\Gamma_{11}^{1}\right)_{y}-\left(\Gamma_{12}^{1}\right)_{x}\right) \frac{\partial}{\partial x}+\left(\left(\Gamma_{11}^{2}\right)_{y}-\left(\Gamma_{12}^{2}\right)_{x}\right) \frac{\partial}{\partial y} \\
& +\Gamma_{11}^{1} \nabla_{\frac{\partial}{\partial x}} \frac{\partial}{\partial y}+\Gamma_{11}^{2} \nabla_{\frac{\partial}{\partial y}} \frac{\partial}{\partial y}-\Gamma_{12}^{1} \nabla_{\frac{\partial}{\partial x}} \frac{\partial}{\partial x}-\Gamma_{12}^{2} \nabla_{\frac{\partial}{\partial x}} \frac{\partial}{\partial y} \\
= & \left(\left(\Gamma_{11}^{1}\right)_{y}-\left(\Gamma_{12}^{1}\right)_{x}+\Gamma_{11}^{2} \Gamma_{22}^{1}-\Gamma_{12}^{2} \Gamma_{12}^{1}\right) \frac{\partial}{\partial x} \\
& +\left(\left(\Gamma_{11}^{2}\right)_{y}-\left(\Gamma_{12}^{1}\right)_{x}+\Gamma_{11}^{1} \Gamma_{12}^{2}+\Gamma_{11}^{2} \Gamma_{22}^{2}-\Gamma_{12}^{1} \Gamma_{11}^{2}-\Gamma_{12}^{2} \Gamma_{12}^{2}\right) \frac{\partial}{\partial y}
\end{aligned}
$$

$\log 0$

$$
\begin{aligned}
K(I)= & \frac{F}{E G-F^{2}}\left(\left(\Gamma_{11}^{1}\right)_{y}-\left(\Gamma_{12}^{1}\right)_{x}+\Gamma_{11}^{2} \Gamma_{22}^{1}-\Gamma_{12}^{2} \Gamma_{12}^{1}\right) \\
& +\frac{G}{E G-F^{2}}\left(\left(\Gamma_{11}^{2}\right)_{y}-\left(\Gamma_{12}^{2}\right)_{x}+\Gamma_{11}^{1} \Gamma_{12}^{2}+\Gamma_{11}^{2} \Gamma_{22}^{2}-\Gamma_{12}^{1} \Gamma_{11}^{2}-\Gamma_{12}^{2} \Gamma_{12}^{2}\right)
\end{aligned}
$$

Além disso, usando (1.17) - (1.20) e (1.26) - (1.31), de forma análoga, podemos observar que na parametrização $(z, \bar{z})$ temos

$$
\begin{aligned}
K(I)= & \frac{\lambda}{|p|^{2}-\lambda^{2}}\left(\left({ }^{\mathbb{C}} \Gamma_{11}^{1}\right)_{\bar{z}}-\left({ }^{\mathbb{C}} \Gamma_{12}^{1}\right)_{z}+{ }^{\mathbb{C}} \Gamma_{11}^{2}{ }^{\mathbb{C}} \Gamma_{22}^{1}-{ }^{\mathbb{C}} \Gamma_{12}^{2}{ }^{\mathbb{C}} \Gamma_{12}^{1}\right) \\
& +\frac{\bar{p}}{|p|^{2}-\lambda^{2}}\left(\left({ }^{\mathbb{C}} \Gamma_{11}^{2}\right)_{\bar{z}}-\left({ }^{\mathbb{C}} \Gamma_{12}^{2}\right)_{z}+{ }^{\mathbb{C}} \Gamma_{11}^{1} \Gamma_{12}^{2}+{ }^{\mathbb{C}} \Gamma_{11}^{2}{ }^{\mathbb{C}} \Gamma_{22}^{2}-{ }^{\mathbb{C}} \Gamma_{12}^{1}{ }^{\mathbb{C}} \Gamma_{11}^{2}-{ }^{\mathbb{C}} \Gamma_{12}^{2}{ }^{\mathbb{C}} \Gamma_{12}^{2}\right) .
\end{aligned}
$$

\subsection{O tensor de Codazzi}

De forma intuitiva podemos dizer que, dada uma superfície $\Sigma \subset \mathbb{R}^{3}$, a geometria intrínseca é aquela que depende da métrica e a extrínseca do endomorfismo de Weingarten $S$. Assim como o tensor curvatura $R$ mede o quanto deixa a métrica de ser plana, veremos como podemos associar de forma natural um tensor ao operador $S$. 
Definição 1.2. Definimos o tensor de Codazzi associado a $S$ como a aplicação $T_{S} \in \Lambda^{2}(S)$ dada por

$$
T_{S}(X, Y)=\nabla_{X} S Y-\nabla_{Y} S X-S[X, Y], \quad X, Y \in \mathcal{X}(\Sigma) .
$$

Vejamos algumas propriedades do Tensor de Codazzi que seguem diretamente da definição.

\section{Proposição 1.1.}

$\operatorname{Seja}\langle,\rangle \in \mathcal{R}(\Sigma)$. Então

1. $T_{S}$ é antisimétrico para qualquer $S \in \mathcal{S}(\Sigma,\langle\rangle$,$) .$

2. $T_{S} \in \tau^{2}(\Sigma)$. Ainda a aplicação

$$
\begin{aligned}
T: \mathcal{S}(\Sigma,\langle,\rangle) & \longrightarrow \tau^{2}(\Sigma) \\
S & \longmapsto T_{S}
\end{aligned}
$$

é uma aplicação $\mathbb{R}$-linear. De fato, satisfaz

$$
T_{f S}(X, Y)=f T_{S}(X, Y)+X(f) S Y-Y(f) S X, \quad X, Y \in \mathcal{X}(\Sigma), f \in C^{\infty}(\Sigma) .
$$

Demonstração. A antisimetria é imediata devido a antisimetria do colchete de Lie. A linearidade com respeito a campos também é imediata devido a linearidade de $S$ e do colchete de Lie. Vejamos como se comporta com respeito a funções $f \in C^{\infty}(\Sigma)$.

$$
\begin{aligned}
T_{S}(f X, Y) & =\nabla_{f X} S Y-\nabla_{Y} S(f X)-S[f X, Y] \\
& =f \nabla_{X} S Y-Y(f) S X-f \nabla_{Y} S X-S(f[X, Y]-Y(f) X) \\
& =f\left(\nabla_{X} S Y-\nabla_{Y} S X-S[X, Y]\right) \\
& =f T_{S}(X, Y) .
\end{aligned}
$$

$\operatorname{Logo} T_{S} \in \tau^{2}(\Sigma)$

Além disso, observamos que

$$
\begin{aligned}
T_{f S}(X, Y) & =\nabla_{X} f S Y-\nabla_{Y} f S X-f S[X, Y] \\
& =f T_{S}(X, Y)+X(f) S Y-Y(f) S X .
\end{aligned}
$$


Notemos que (1.43) nos permite estender o Tensor de Codazzi a funções do seguinte modo: tomemos $f \in C^{\infty}(\Sigma)$ e consideremos $S=f I d \in \mathcal{S}(\Sigma,\langle\rangle$,$) . Então, como$ $\nabla$ é livre de torção e $T_{I d}=0$, temos que

$$
T_{f}(X, Y)=X(f) Y-Y(f) X
$$

A motivação da definição deste tensor surge da teoria de superfícies em formas espaciais. Neste caso, se temos uma superfície $\Sigma \subset \mathbb{R}^{3}$, sabe-se que o operador de Weingarten de tais superfícies satisfaz a equação de Codazzi, isto é,

$$
\nabla_{X} S Y-\nabla_{Y} S X-S[X, Y]=0, \quad X, Y \in \mathcal{X}(\Sigma)
$$

ou equivalentemente, pela definição anterior,

$$
T_{S}(X, Y)=0, \quad X, Y \in \mathcal{X}(\Sigma)
$$

Logo, de forma natural, definimos, seguindo a notação empregada

Definição 1.3. Seja $(\langle\rangle, S,) \in \mathcal{P}(\Sigma)$. Então diremos que $S$ é de Codazzi com respeito a $\langle$,$\rangle (ou que (\langle\rangle, S$,$) é de Codazzi) se$

$$
T_{S}(X, Y)=0, \quad X, Y \in \mathcal{X}(\Sigma)
$$

Além disso, denotaremos por $\mathcal{C}(\Sigma) \subset \mathcal{P}(\Sigma)$, chamado conjunto de Pares de Codazzi, ao subconjunto de pares fundamentais cujo endomorfismo autoadjunto é de Codazzi.

O Tensor de Codazzi, de forma intuitiva, mede o quanto deixa um par fundamental de ser de Codazzi.

A partir de agora, escreveremos $\partial_{x}$ no lugar de $\frac{\partial}{\partial_{x}}$.

Vejamos como ficam as equações anteriores quando trabalhamos numa vizinhança coordenada. Dados $(x, y)$ parâmetros locais sobre $U \subset \Sigma$ e usando as propriedades vistas do Tensor de Codazzi, é suficiente conhecer como atua $T_{S}$ sobre os campos fundamentais $\left\{\partial_{x}, \partial_{y}\right\}$. Assim, dado $(\langle\rangle, S,) \in \mathcal{P}(\Sigma)$ temos

$$
T_{S}\left(\partial_{x}, \partial_{y}\right)=a \partial_{x}+b \partial_{y}
$$


para certos $a, b \in C^{\infty}(U)$. Logo de (1.2) e (1.3)

$$
\begin{aligned}
\left\langle T_{S}\left(\partial_{x}, \partial_{y}\right), \partial_{x}\right\rangle & =\left\langle\nabla_{\partial_{x}} S \partial_{y}-\nabla_{\partial_{y}} S \partial_{x}, \partial_{x}\right\rangle \\
& =\partial_{x}\left\langle S \partial_{y}, \partial_{x}\right\rangle-\left\langle S \partial_{y}, \nabla_{\partial_{x}} \partial_{x}\right\rangle-\partial_{y}\left\langle S \partial_{x}, \partial_{x}\right\rangle+\left\langle S \partial_{x}, \nabla_{\partial_{y}} \partial_{x}\right\rangle \\
& =f_{x}-\left\langle S \partial_{y}, \Gamma_{11}^{1} \partial_{x}+\Gamma_{11}^{2} \partial_{y}\right\rangle-e_{x}+\left\langle S \partial_{x}, \Gamma_{12}^{1} \partial_{x}+\Gamma_{12}^{2} \partial_{y}\right\rangle \\
& =f_{x}-e_{y}+e \Gamma_{12}^{1}+f\left(\Gamma_{12}^{2}-\Gamma_{11}^{1}\right)-g \Gamma_{11}^{2}
\end{aligned}
$$

De forma análoga

$$
\left\langle T_{S}\left(\partial_{x}, \partial_{y}\right), \partial_{y}\right\rangle=g_{x}-f_{y}+e \Gamma_{22}^{1}+f\left(\Gamma_{22}^{2}-\Gamma_{12}^{1}\right)-g \Gamma_{12}^{2}
$$

Isto é, para parâmetros locais quaisquer $(x, y)$ em que o par fundamental $(I, I I)$ vem dado por (1.2) e (1.3) se verifica

$$
\begin{aligned}
& \left\langle T_{S}\left(\partial_{x}, \partial_{y}\right), \partial_{y}\right\rangle=g_{x}-f_{y}+e \Gamma_{22}^{1}+f\left(\Gamma_{22}^{2}-\Gamma_{12}^{1}\right)-g \Gamma_{12}^{2}, \\
& \left\langle T_{S}\left(\partial_{x}, \partial_{y}\right), \partial_{x}\right\rangle=f_{x}-e_{y}+e \Gamma_{12}^{1}+f\left(\Gamma_{12}^{2}-\Gamma_{11}^{1}\right)-g \Gamma_{11}^{2}
\end{aligned}
$$

Consequentemente, $S \in \mathcal{S}(\Sigma,\langle\rangle$,$) é de Codazzi se, e somente se, as equações$ (1.44) e (1.45) são identicamente nulas. Estas equações são conhecidas como as equações de Codazzi.

De forma análoga, podemos reescrever as equações anteriores usando um parâmetro local complexo $z=x+i y$, em que o par fundamental $(I, I I)$ vem dado por (1.15) e (1.16). Desta forma

$$
\begin{aligned}
& \left\langle T_{S}\left(\partial_{z}, \partial_{\bar{z}}\right), \partial_{\bar{z}}\right\rangle=\bar{q}_{z}-\rho_{\bar{z}}+q^{\mathbb{C}} \Gamma_{22}^{1}+\rho\left({ }^{\mathbb{C}} \Gamma_{22}^{2}-{ }^{\mathbb{C}} \Gamma_{12}^{1}\right)-\bar{q}^{\mathbb{C}} \Gamma_{12}^{2} \\
& \left\langle T_{S}\left(\partial_{z}, \partial_{\bar{z}}\right), \partial_{z}\right\rangle=-q_{\bar{z}}+\rho_{z}+q^{\mathbb{C}} \Gamma_{12}^{1}+\rho\left({ }^{\mathbb{C}} \Gamma_{12}^{2}-{ }^{\mathbb{C}} \Gamma_{11}^{1}\right)-\bar{q}^{\mathbb{C}} \Gamma_{11}^{2}
\end{aligned}
$$

Exemplo 1.1. Alguns pares de Codazzi surgem de forma natural no estudo de superfícies. Por exemplo, a primeira e segunda formas fundamentais de uma superfície isometricamente imersa em uma forma espacial tridimensional constitui um par de Codazzi. O mesmo ocorre para superfícies tipo espaço em uma forma espacial Lorentziana tridimensional. Mais geralmente, se uma superfície é imersa em uma forma espacial $n$-dimensional (semiRiemanniana) e possui um campo unitário normal $N$, então a métrica induzida e sua segunda forma fundamental associada com $N$ constitui um par de Codazzi. 


\subsection{Parametrizações especiais}

Sobre uma superfície podemos obter uma série de parametrizações especiais onde é mais fácil trabalhar. Dentre elas estão as parametrizações por parâmetros isotérmicos, duplamente ortogonais e os assintóticos. Assim, o que faremos agora é particularizar as equações anteriores para um par fundamental $(I, I I)$ para estes tipos de parametrizações. Observe que as equações a seguir são válidas para superfícies.

\subsubsection{Parâmetros isotérmicos}

Uma parametrização $(s, t)$, com a orientação induzida da superfície em uma vizinhança $U \subset \Sigma$, é dita isotérmica se para uma métrica Riemanniana $I$ dada por (1.2) se verifica

$$
E=G>0 \text { e } F=0
$$

ou visto no parâmetro complexo $z=s+i t$, chamada parâmetro conforme, se para uma métrica Riemanniana $I$. dada por (1.15), verifica-se

$$
p=0 .
$$

Sabe-se que tais parâmetros sempre existem localmente para qualquer métrica Riemanniana e que podemos recobrir a variedade com este tipo de vizinhanças coordenadas. Ainda, a mudança de carta entre vizinhanças isotérmicas positivamente orientadas é holomorfa e portanto podemos ver $\Sigma$ como uma superfície de Riemann.

Quando trabalharmos com parâmetros isotérmicos, sempre o faremos usando o parâmetro complexo associado $z$, por isso veremos as equações para esta parametrização.

\section{Proposição 1.2.}

Seja $(I, I I) \in \mathcal{P}(\Sigma)$ e z um parâmetro conforme para I. Então

$$
\begin{aligned}
I & =2 \lambda|d z|^{2} \\
I I & =q d z^{2}+2 H \lambda|d z|^{2}+\bar{q} d \bar{z}^{2}
\end{aligned}
$$


e se verificam as seguintes equações

$$
\begin{aligned}
K & =H^{2}-\frac{|q|^{2}}{\lambda^{2}} \\
S \partial_{z} & =H \partial_{z}+\frac{q}{\lambda} \partial_{\bar{z}} \\
\Gamma_{11}^{1} & =\frac{\lambda_{z}}{\lambda} \\
\Gamma_{11}^{2} & =0 \\
\Gamma_{12}^{1} & =0 \\
T_{S}\left(\partial_{z}, \partial_{\bar{z}}\right) & =T_{H}\left(\partial_{z}, \partial_{\bar{z}}\right)+\frac{1}{\lambda}\left(\bar{q}_{z} \partial_{z}+q_{\bar{z}} \partial_{\bar{z}}\right), \\
\left\langle T_{S}\left(\partial_{z}, \partial_{\bar{z}}\right), \partial_{z}\right\rangle & =\lambda H_{z}-q_{\bar{z}}, \\
K(I) & =-\frac{1}{\lambda}(\ln \lambda)_{z \bar{z}} .
\end{aligned}
$$

Demonstração. Já que z é um parâmetro conforme para $I$, temos que $p=0$ e, portanto, de (1.21), a curvatura média do $\operatorname{par}(I, I I)$ é dada por $H=\frac{\rho}{\lambda}$. Assim, $\rho=H \lambda$. De onde obtemos (1.48) e (1.49) imediatamente.

Seja $S$ o operador associado a $I I$. Vejamos como atua $S$ sobre campos; em particular, somente necessitamos conhecer como atua sobre o campo $\partial_{z}$. Se tomarmos

$$
S \partial_{z}=a \partial_{z}+b \partial_{\bar{z}}
$$

então já que

$$
\begin{aligned}
q & =\left\langle S \partial_{z}, \partial_{z}\right\rangle=b \lambda, \\
\lambda H & =\left\langle S \partial_{z}, \partial_{\bar{z}}\right\rangle=a \lambda
\end{aligned}
$$

obtemos (1.51).

De (1.32) - (1.34), considerando $p=0$, obtemos (1.52) - (1.54).

Uma vez que tenhamos os símbolos de Christoffel, é fácil ver que

$$
\begin{aligned}
T_{S}\left(\partial_{z}, \partial_{\bar{z}}\right)= & \frac{1}{\lambda}\left((H \lambda)_{z}-q_{\bar{z}}-H \lambda \Gamma_{11}^{1}\right) \partial_{\bar{z}} \\
& -\frac{1}{\lambda}\left((H \lambda)_{\bar{z}}-\bar{q}_{z}-H \lambda \Gamma_{22}^{2}\right) \partial_{z} \\
= & H_{z} \partial_{\bar{z}}-H_{\bar{z}} \partial_{z}+\frac{1}{\lambda}\left(\bar{q}_{z} \partial_{z}-q_{\bar{z}} \partial_{\bar{z}}\right) \\
= & T_{H}\left(\partial_{z}, \partial_{\bar{z}}\right)+\frac{1}{\lambda}\left(\bar{q}_{z} \partial_{z}-q_{\bar{z}} \partial_{\bar{z}}\right),
\end{aligned}
$$

isto é, (1.55) se verifica. É imediato verificar (1.56) tomando o produto escalar de $\partial_{z}$ com $(1.55)$. 
Por último, a equação da curvatura de Gauss é imediata de (1.42), a partir dos símbolos de Christoffel e $p=0$.

Conforme comentamos anteriormente, o parâmetro conforme é sempre possível consegui-lo quando temos uma métrica Riemanniana sobre uma superfície. Assim, se $(I, I I) \in \mathcal{P}(\Sigma)$, onde $I I$ é uma métrica Riemanniana, também podemos considerar um parâmetro conforme $z$ para $I I$. Neste caso o que teríamos é $q=0$ em (1.16). Note ainda que, devido à $I I$ ser Riemanniana, temos $K(I, I I)>0$. Para este parâmetro obtemos:

\section{Proposição 1.3.}

Seja $(I, I I) \in \mathcal{P}(\Sigma)$, onde II é Riemanniana. Seja $S^{I} \in \mathcal{S}(\Sigma, I I)$ o endomorfismo autoadjunto associado a I com respeito a II e $S^{I I} \in \mathcal{S}(\Sigma, I)$ o endomorfismo autoadjunto associado a II com respeito a I. Seja z um parâmetro conforme para II, isto é,

$$
\begin{aligned}
I & =p d z^{2}+2 \lambda|d z|^{2}+\bar{p} d \bar{z}^{2}, \\
I I & =2 \rho|d z|^{2}
\end{aligned}
$$

e denotemos

$$
D=|p|^{2}-\lambda^{2}
$$

Então se verificam as seguintes equações

$$
\begin{aligned}
\lambda K & =\rho H \\
S^{I} \partial_{z} & =\frac{H}{K} \partial_{z}+\frac{p}{\rho} \partial \bar{z} \\
S^{I I} \partial_{z} & =K\left(\frac{H}{K} \partial_{z}-\frac{p}{\rho} \partial_{\bar{z}}\right), \\
\Gamma_{11}^{1}+\Gamma_{12}^{2} & =\frac{D_{z}}{2 D} \\
T_{S^{I I}}\left(\partial_{z}, \partial_{\bar{z}}\right) & =\frac{K_{z}}{2} S^{I} \partial_{\bar{z}}-\frac{K_{\bar{z}}}{2} S^{I} \partial_{z}-\frac{(K \bar{p})_{z}}{\rho} \partial_{z}+\frac{(K p)_{\bar{z}}}{\rho} \partial_{\bar{z}} \\
\left\langle T_{S^{I I}}\left(\partial_{z}, \partial_{\bar{z}}\right), \partial_{z}\right\rangle & =2 \rho\left(\frac{K_{z}}{4 K}+\Gamma_{12}^{2}\right) .
\end{aligned}
$$

Demonstração. A primeira equação (1.60) obtém-se diretamente das definições de $H$ e $K$, (1.21) e (1.22), fazendo $q=0$. Agora, para obter (1.61), se escrevermos $S^{I} \partial_{z}=a \partial_{z}+b \partial_{\bar{z}}$ para certas funções locais $a, b$, teremos de (1.58) e (1.59) que

$$
\begin{aligned}
& p=I I\left(S^{I} \partial_{z}, \partial_{z}\right)=b \rho, \\
& \lambda=I I\left(S^{I} \partial_{z}, \partial_{\bar{z}}\right)=a \rho .
\end{aligned}
$$


Usando (1.60), resulta a expressão desejada. Analogamente, se $S^{I I} \partial_{z}=a \partial_{z}+b \partial_{\bar{z}}$

$$
\begin{aligned}
& 0=I\left(S^{I I} \partial_{z}, \partial_{z}\right)=a p+b \lambda, \\
& \rho=I\left(S^{I I} \partial_{z}, \partial_{\bar{z}}\right)=a \lambda+b \bar{p} .
\end{aligned}
$$

De onde obtemos, substituindo $a$ e $b,(1.62)$.

A equação que envolve os símbolos de Christoffel é geral, isto é, não depende de que o parâmetro $z$ seja conforme para $I I$, como se pode ver de forma direta somando ambos os símbolos das equações (1.32) e (1.35).

A partir de $D=|p|^{2}-\lambda^{2}$, podemos reescrever a curvatura extrínseca como

$$
K=-\frac{\rho^{2}}{D}
$$

Derivando (1.66) em relação a $z$, obtemos a seguinte igualdade

$$
\rho \frac{K_{z}}{2 K}=\rho_{z}+\rho \frac{D_{z}}{2 D}
$$

A equação (1.65) segue de (1.47), usando esta última equação e (1.63), logo

$$
\begin{aligned}
\left\langle T_{S^{I I}}\left(\partial_{z}, \partial_{\bar{z}}\right), \partial_{z}\right\rangle & =\rho_{z}+\rho\left(\Gamma_{12}^{2}-\Gamma_{11}^{1}\right) \\
& =\rho_{z}-\rho \frac{D_{z}}{2 D}+2 \rho \Gamma_{12}^{2} \\
& =\rho \frac{K_{z}}{2 K}+2 \rho \Gamma_{12}^{2} \\
& =2 \rho\left(\frac{K_{z}}{4 K}+\Gamma_{12}^{2}\right) .
\end{aligned}
$$

Denotando $\alpha=\left\langle T_{S^{I I}}\left(\partial_{z}, \partial_{\bar{z}}\right), \partial_{z}\right\rangle$, podemos concluir de (1.46),(1.47) e (1.29) (1.31) que $-\bar{\alpha}=\left\langle T_{S^{I I}}\left(\partial_{z}, \partial_{\bar{z}}\right), \partial_{\bar{z}}\right\rangle$.

\subsubsection{Parâmetros duplamente ortogonais}

Seja $(I, I I)$ um par fundamental sobre uma superfície $\Sigma$ e $p \in \Sigma^{\prime}$, sendo $\Sigma^{\prime}$ o conjunto formado pelo interior do conjunto de pontos umbílicos e o conjunto de pontos não umbílicos. Então, existem parâmetros locais conhecidos comoduplamente ortogonais, isto é, parâmetros em que $F=f=0$,

$$
\begin{gathered}
I=E d u^{2}+G d v^{2}, \\
I I=e d u^{2}+g d v^{2} .
\end{gathered}
$$


Como $\Sigma^{\prime}$ é um conjunto denso em toda a superfície, usaremos que certas propriedades são válidas para parâmetros duplamente ortogonais e deduziremos que essas propriedades são válidas em toda a superfície por continuidade.

É importante não esquecer que os parâmetros anteriores $(u, v)$ da parametrização por linhas de curvatura, isto é, $e=k_{1} E$ e $g=k_{2} G$, onde $k_{1}$ e $k_{2}$ são as curvaturas principais do par $(I, I I)$. Neste caso, se $S$ é o endomorfismo autoadjunto associado a $I I$ e $\left\{\partial_{u}, \partial_{v}\right\}$ são os campos coordenados associadas a parametrização $(u, v)$, então

$$
S \partial_{u}=k_{1} \partial_{u}, \quad S \partial_{v}=k_{2} \partial_{v}
$$

Vejamos agora como ficam as equações nesta parametrização

\section{Proposição 1.4 .}

Sejam $(I, I I) \in \mathcal{P}(\Sigma), p \in \Sigma^{\prime} e(u, v)$ parâmetros locais duplamente ortogonais,

$$
\begin{gathered}
I=E d u^{2}+G d v^{2}, \\
I I=k_{1} E d u^{2}+k_{2} G d v^{2} .
\end{gathered}
$$

Então se verificam as seguintes equações

$$
\begin{aligned}
a & =\left(\frac{k_{1}-k_{2}}{2}\right)^{2} \\
\Gamma_{11}^{1} & =\frac{E_{u}}{2 E}, \\
\Gamma_{11}^{2} & =-\frac{E_{v}}{2 G} \\
\Gamma_{12}^{1} & =\frac{E_{v}}{2 E} \\
\Gamma_{12}^{2} & =\frac{G_{u}}{2 G}, \\
\Gamma_{22}^{1} & =-\frac{G_{u}}{2 E}, \\
\Gamma_{22}^{2} & =\frac{G_{v}}{2 G}, \\
T_{S}\left(\partial_{u}, \partial_{v}\right) & =T_{H}\left(\partial_{u}, \partial_{v}\right)-\sqrt{a}\left((\ln (\sqrt{a} E))_{u} \partial_{v}+(\ln (\sqrt{q} G))_{v} \partial_{u}\right) \\
\left\langle T_{S}\left(\partial_{u}, \partial_{v}\right), \partial_{u}\right\rangle & =\left(k_{1}\right)_{v}+\sqrt{a} \frac{E_{v}}{E}, \\
\left\langle T_{S}\left(\partial_{u}, \partial_{v}\right), \partial_{v}\right\rangle & =-\left(k_{2}\right)_{u}+\sqrt{a} \frac{G_{u}}{G}, \\
K(I) & =-\frac{1}{2 \sqrt{E G}}\left(\left(\frac{E_{v}}{\sqrt{E G}}\right)_{v}+\left(\frac{E_{u}}{\sqrt{E G}}\right)_{u}\right) .
\end{aligned}
$$


Demonstração. Da expressão do par em coordenadas duplamente ortogonais, (1.67) e (1.68), obtemos diretamente (1.69). Os símbolos de Christoffel são facilmente calculados de (1.6) - (1.11), usando (1.67). Por outro lado,

$$
\begin{aligned}
T_{S}\left(\partial_{u}, \partial_{v}\right) & =\nabla_{\partial_{u}} S \partial_{v}-\nabla_{\partial_{v}} S \partial_{u} \\
& =\left(k_{2}\right)_{u} \partial_{v}-\left(k_{1}\right)_{v} \partial_{u}-\left(k_{1}-k_{2}\right) \nabla_{\partial_{u}} \partial_{v} \\
& =H_{u} \partial_{v}-H_{v} \partial_{u}-(\sqrt{a})_{u} \partial_{v}-(\sqrt{a})_{v} \partial_{u}-2 \sqrt{a} \nabla_{\partial_{u}} \partial_{v} \\
& =T_{H}\left(\partial_{u}, \partial_{v}\right)-\sqrt{a}\left((\ln (\sqrt{a} E))_{u} \partial_{v}+(\ln (\sqrt{a} G))_{v} \partial_{u}\right),
\end{aligned}
$$

como queríamos demonstrar. As equações (1.77) e (1.78) são obtidas diretamente desta última. Finalmente, (1.79) é um cálculo direto.

\subsubsection{Parâmetros assintóticos}

Como vimos na Subseção 1.3.1, quando $I I \in \mathcal{R}(\Sigma)$ podemos considerar um parâmetro conforme para esta métrica Riemanniana. Assim, mesmo quando $I I \in \mathcal{L}(\Sigma)$ e portanto $K<0$, podemos obter parâmetros locais reais $(x, y)$ para os quais $e=g=0$, isto é

$$
\begin{aligned}
I & =E d x^{2}+2 F d x d y+G d y^{2}, \\
I I & =2 f d x d y
\end{aligned}
$$

que são conhecidos como parâmetros assintóticos. De maneira intuitiva, podemos pensar em tal parametrização como fizemos em parâmetros conformes $(z, \bar{z})$ para uma métrica Riemanniana. Isto é, em relação aos cálculos, o parâmetro $x$ faz o papel de $z$ e o parâmetro $y$ o papel de $\bar{z}$.

Na proposição seguinte, consideraremos operadores autoadjuntos associados a $I I$, que será uma métrica Lorentziana. Isso não é problema, embora tudo o que foi discutido acima está associado uma métrica Riemanniana para os operadores, uma vez que o que realmente precisamos é que a métrica não degenere, independentemente do índice da métrica.

\section{Proposição 1.5.}

Seja $(I, I I) \in \mathcal{P}(\Sigma)$ onde II é Lorentziana. Suponhamos que existe $S^{I} \in \mathcal{S}(\Sigma, I I)$ endomorfismo autoadjunto associado a I com respeito a II, isto é,

$$
I(X, Y)=I I\left(S^{I} X, Y\right), \quad X, Y \in \mathcal{X}(\Sigma)
$$


e $S^{I I} \in \mathcal{S}(\Sigma, I)$ o endomorfismo autoadjunto associado a II com respeito a $I$, isto é,

$$
I I(X, Y)=I\left(S^{I I} X, Y\right), \quad X, Y \in \mathcal{X}(\Sigma)
$$

Seja $(x, y)$ parâmetros assintóticos para $(I, I I)$, isto é

$$
\begin{aligned}
I & =E d x^{2}+2 F d x d y+G d y^{2} \\
I I & =2 f d x d y
\end{aligned}
$$

e denotemos por

$$
D=E G-F^{2}
$$

Então se verificam as seguintes equações

$$
\begin{aligned}
F K & =f H \\
S^{I} \partial_{x} & =\frac{H}{K} \partial_{x}+\frac{E}{f} \partial_{y} \\
S^{I} \partial_{x} & =\frac{G}{f} \partial_{x}+\frac{H}{K} \partial_{y} \\
S^{I I} \partial_{x} & =K\left(\frac{H}{K} \partial_{x}-\frac{E}{f} \partial_{y}\right) \\
S^{I I} \partial_{y} & =K\left(-\frac{G}{f} \partial_{x}+\frac{H}{K} \partial_{y}\right) \\
\Gamma_{11}^{1}+\Gamma_{12}^{2} & =\frac{D_{x}}{2 D}, \\
\Gamma_{12}^{1}+\Gamma_{22}^{2} & =\frac{D_{y}}{2 D}, \\
T_{S^{I I}}\left(\partial_{x}, \partial_{y}\right) & =\frac{K_{x}}{2} S^{I} \partial_{y}-\frac{K_{y}}{2} S^{I} \partial_{x}-\frac{(K G)_{x}}{f} \partial_{x}+\frac{(K E)_{y}}{f} \partial_{y} \\
\left\langle T_{S^{I I}}\left(\partial_{x}, \partial_{y}\right), \partial_{x}\right\rangle & =2 f\left(\frac{K_{x}}{4 K}+\Gamma_{12}^{2}\right) \\
\left\langle T_{S^{I I}}\left(\partial_{x}, \partial_{y}\right), \partial_{y}\right\rangle & =-2 f\left(\frac{K_{y}}{4 K}+\Gamma_{12}^{1}\right) .
\end{aligned}
$$

Demonstração. Análoga a demonstração da proposição 1.4 .

\subsection{Formas Espaciais}

Descreveremos nesta seção as formas espaciais, que são as variedades de curvatura seccional constante. Embora vamos apresentar também o espaço euclidiano, neste trabalho, vamos lidar apenas com a esfera euclidiana e o espaço hiperbólico. 
Seja $\mathbb{M}^{n+1}(c)$ uma variedade Riemanniana de dimensão $n+1, n \geq 1$, completa, simplesmente conexa e com curvatura seccional constante $c$. Então o Teorema de Cartan nos diz que, a menos de isometrias, $\mathbb{M}^{n+1}(c)$ é uma das seguintes variedades:

\subsubsection{O espaço Euclidiano}

Se $c=0, \mathbb{M}^{n+1}(0)$ é o espaço euclidiano $(n+1)$-dimensional usual $\mathbb{R}^{n+1}$, com a métrica

$$
\langle,\rangle=\sum_{i=1}^{n+1} d x_{i}^{2}
$$

\subsubsection{A esfera Euclidiana}

Se $c>0, \mathbb{M}^{n+1}(c)$ é a esfera $(n+1)$-dimensional usual de raio $\frac{1}{\sqrt{c}}$, isto é,

$$
\mathbb{S}^{n+1}(c)=\left\{\left(x_{0}, x_{1}, \ldots, x_{n+1}\right) \in \mathbb{R}^{n+2}: \sum_{i=0}^{n+1} x_{i}^{2}=\frac{1}{c}\right\}
$$

com a métrica induzida $\langle\rangle=,\sum_{i=0}^{n+1} d x_{i}^{2}$, de $\mathbb{R}^{n+2}$.

\subsubsection{O espaço hiperbólico}

Este é o caso em que $c<0$. Para este espaço veremos três modelos diferentes.

\section{O hiperbolóide de uma folha}

Se $c<0, \mathbb{M}^{n+1}(c)$ é o espaço hiperbólico $(n+1)$-dimensional usual, isto é, o conjunto de $\mathbb{L}^{n+2}$ dado por

$$
\mathbb{H}^{n+1}(c)=\left\{\left(x_{0}, x_{1}, \ldots, x_{n+1}\right) \in \mathbb{L}^{n+2}:-x_{0}^{2}+\sum_{i=1}^{n+1} x_{i}^{2}=\frac{1}{c}, x_{0}>0\right\}
$$

com a métrica induzida $\langle\rangle=,-d x_{0}^{2}+\sum_{i=0}^{n+1} d x_{i}^{2}$ de $\mathbb{L}^{n+2}$.

\section{Modelo do semiespaço superior}

Consideremos o subconjunto de $\mathbb{R}^{n+1}$ dado por

$$
\mathbb{R}_{+}^{n+1}=\left\{\left(x_{1}, \ldots, x_{n+1}\right) \in \mathbb{R}^{n+1}: x_{n+1}>0\right\}
$$


provido com a métrica

$$
d s^{2}=\frac{1}{-c x_{n+1}^{2}}\left(d x_{1}^{2}+\cdots+d x_{n+1}^{2}\right)
$$

\section{Modelo conforme da bola ou modelo de Poincaré}

Consideremos sobre a bola aberta centrada na origem de $\mathbb{R}^{n+1}$ e raio $\frac{1}{\sqrt{-c}}, \mathbb{B}\left(\frac{1}{\sqrt{-c}}\right)$, a métrica de curvatura seccional constante $c$ dada por

$$
d s^{2}=\frac{4}{\left(1+c|x|^{2}\right)^{2}}\left(d x_{1}^{2}+\cdots+d x_{2}^{2}\right),
$$

onde $x=\left(x_{1}, \ldots, x_{n}\right)$ e $|\cdot|$ é a norma Euclidiana de $\mathbb{R}^{n}$.

\subsection{Equações fundamentais}

Nesta seção vamos deduzir algumas equações que utilizaremos ao longo de todo o trabalho.

Seja $f: M \rightarrow \bar{M}$ uma imersão de uma variedade diferenciável $M$ de dimensão $n$ em uma variedade Riemanniana $\bar{M}$ de dimensão igual a $k=n+m$. Vamos considerar em $M$ a métrica induzida por $\bar{M}$, isto é, sejam $v_{1}, v_{2} \in T_{p} M$, definimos $\left\langle v_{1}, v_{2}\right\rangle=\left\langle d f_{p}\left(v_{1}\right), d f_{p}\left(v_{2}\right)\right\rangle$. Assim $f$ passa a ser uma imersão isométrica de $M$ em $\bar{M}$. Veremos como se relacionam as geometrias de $M$ e $\bar{M}$.

Como $f$ é uma imersão, para cada $p \in M$, existe uma vizinhança $U \subset M$ de $p$ tal que $f(U) \subset \bar{M}$ é uma subvariedade de $\bar{M}$. Isto quer dizer que existem uma vizinhança $\bar{U} \subset \bar{M}$ de $f(p)$ e um difeomorfismo $\varphi: \bar{U} \rightarrow V \subset \mathbb{R}^{k}$ em um aberto $V$ do $\mathbb{R}^{k}$, tais que $\varphi$ aplica difeomorficamente $f(U) \subset \bar{U}$ em um aberto do subespaço $\mathbb{R}^{n} \subset \mathbb{R}^{k}$.

Identificaremos $U \operatorname{com} f(U)$ e cada vetor $v \in T_{q} M, q \in U, \operatorname{com} d f_{q}(v) \in T_{f(q)} \bar{M}$. Usaremos tais identificações para estender, por exemplo, um campo local de vetores de $M$ a um campo local de vetores em $\bar{M}$. Se $U$ é suficientemente pequeno, tal extensão é sempre possível.

Para cada $p \in M$, o produto interno em $T_{p} \bar{M}$ decompõe $T_{p} \bar{M}$ na soma direta

$$
T_{p} \bar{M}=T_{p} M \oplus\left(T_{p} M\right)^{\perp}
$$

onde $\left(T_{p} M\right)^{\perp}$ é o complemento ortogonal de $T_{p} M$ em $T_{p} \bar{M}$. 
Se $v \in T_{p} \bar{M}, p \in M$, podemos escrever

$$
v=v^{T}+v^{N}, \quad v^{T} \in T_{p} M, \quad v^{N} \in\left(T_{p} M\right)^{\perp} .
$$

Denominamos $v^{T}$ a componente tangencial de $v$ e $v^{N}$ a componente normal de $v$.

A conexão Riemanniana de $\bar{M}$ será indicada por $\bar{\nabla}$. Se $X$ e $Y$ são campos locais de vetores em $M$, e $\bar{X}, \bar{Y}$ são extensões locais a $\bar{M}$, definimos

$$
\nabla_{X} Y=\left(\bar{\nabla}_{\bar{X}} \bar{Y}\right)^{T}
$$

Pode-se verificar que esta é a conexão Riemanniana relativa a métrica induzida de $M$.

\subsubsection{Segunda forma fundamental}

Se $X, Y$ são campos locais em $M$,

$$
B(X, Y)=\bar{\nabla}_{\bar{X}} \bar{Y}-\nabla_{X} Y
$$

é um campo local em $\bar{M}$ normal a $M$. Pode-se verificar que $B(X, Y)$ não depende das extensões $\bar{X}, \bar{Y}$ e que é uma aplicação bilinear e simétrica. Como $B$ é bilinear, concluímos, exprimindo $B$ em um sistema de coordenadas, que o valor de $B(X, Y)(p)$ depende apenas de $X(p)$ e $Y(p)$.

Agora podemos definir a segunda forma fundamental. Seja $p \in M$ e $\eta \in\left(T_{p} M\right)^{\perp}$. A aplicação $H_{\eta}: T_{p} M \times T_{p} M \rightarrow \mathbb{R}$ dada por

$$
H_{\eta}(x, y)=\langle B(x, y), \eta\rangle, \quad x, y \in T_{p} M
$$

é uma forma bilinear simétrica.

Definição 1.4. A forma quadrática $I I_{\eta}$ definida em $T_{p} M$ por

$$
I I_{\eta}(x)=H_{\eta}(x, x)
$$

é chamada a segunda forma fundamental de $f$ em $p$ segundo o vetor normal $\eta$.

Observe que à aplicação bilinear $H_{\eta}$ fica associada uma aplicação linear autoadjunta $S_{\eta}: T_{p} M \rightarrow T_{p} M$ por

$$
\left\langle S_{\eta}(x), y\right\rangle=H_{\eta}(x, y)=\langle B(x, y), \eta\rangle
$$


A proposição seguinte nos dá uma expressão da aplicação linear associada à segunda forma fundamental em termos da derivada covariante.

\section{Proposição 1.6.}

Seja $p \in M, x \in T_{p} M$ e $\eta \in\left(T_{p} M\right)^{\perp}$. Seja $N$ uma extensão local de $\eta$ normal a $M$. Então

$$
S_{\eta}(x)=-\left(\bar{\nabla}_{x} N\right)^{T}
$$

Demonstração. Seja $y \in T_{p} M$ e $X, Y$ extensões locais de $x, y$, respectivamente, e tangente a $M$. Então $\langle N, Y\rangle=0$ de onde $\left\langle\bar{\nabla}_{X} N, Y\right\rangle+\left\langle N, \bar{\nabla}_{X} Y\right\rangle=0$, e portanto

$$
\begin{aligned}
\left\langle S_{\eta}(x), y\right\rangle & =\langle B(X, Y)(p), N\rangle=\left\langle\bar{\nabla}_{X} Y-\nabla_{X} Y, N\right\rangle(p) \\
& =\left\langle\bar{\nabla}_{X} Y, N\right\rangle(p)=-\left\langle Y, \bar{\nabla}_{X} N\right\rangle(p)=\left\langle-\bar{\nabla}_{x} N, y\right\rangle
\end{aligned}
$$

para todo $y \in T_{p} M$.

\subsubsection{As equações fundamentais de uma imersão isométrica}

No que se segue, usaremos sistematicamente as letras latinas $X, Y, Z$, etc., para indicar os campos diferenciáveis de vetores tangentes e as letras gregas $\xi, \eta, \zeta$, etc., para indicar os campos diferenciáveis de vetores normais. Dados $X$ e $\eta$, já vimos que a componente tangente de $\bar{\nabla}_{X} \eta$ é dada por $\left(\bar{\nabla}_{X} \eta\right)^{T}=-S_{\eta} X$. Passaremos agora a estudar a componente normal de $\bar{\nabla}_{X} \eta$, que será chamada a conexão normal $\nabla^{\perp}$ da imersão. Explicitamente,

$$
\nabla_{X}^{\perp} \eta=\left(\bar{\nabla}_{X} \eta\right)^{N}=\bar{\nabla}_{X} \eta-\left(\bar{\nabla}_{X} \eta\right)^{T}=\bar{\nabla}_{X} \eta+S_{\eta} X
$$

Pode-se verificar que a conexão normal $\nabla^{\perp}$ possui as propriedades usuais de uma conexão, isto é, é linear em $X$, aditiva em $\eta$, e

$$
\nabla_{X}^{\perp}(f \eta)=f \nabla_{X}^{\perp} \eta+X(f) \eta, \quad f \in C^{\infty}(M)
$$

De maneira análoga ao caso do fibrado tangente, introduz-se a partir de $\nabla^{\perp}$ uma noção de curvatura no fibrado normal que é chamada curvatura normal $R^{\perp}$ da imersão e definida por

$$
R^{\perp}(X, Y) \eta=\nabla_{Y}^{\perp} \nabla_{X}^{\perp} \eta-\nabla_{X}^{\perp} \nabla_{Y}^{\perp} \eta+\nabla_{[X, Y]}^{\perp} \eta
$$

Assim a geometria da imersão de decompõe em duas geometrias: uma geometria do fibrado tangente e uma geometria do fibrado normal. 


\section{Proposição 1.7.}

As seguintes equações se verificam:

(a) Equação de Gauss

$$
\langle\bar{R}(X, Y) Z, T\rangle=\langle R(X, Y) Z, T\rangle+\langle B(X, T), B(Y, Z)\rangle-\langle B(Y, T), B(X, Z)\rangle .
$$

(b) Equação de Ricci

$$
\langle\bar{R}(X, Y) \eta, \xi\rangle=\left\langle R^{\perp}(X, Y) \eta, \xi\right\rangle+\left\langle S_{\eta}\left(S_{\xi} X\right), Y\right\rangle-\left\langle S_{\xi}\left(S_{\eta} X\right), Y\right\rangle .
$$

Demonstração. Note que

$$
\begin{aligned}
\bar{R}(X, Y) Z= & \bar{\nabla}_{Y} \bar{\nabla}_{X} Z-\bar{\nabla}_{X} \bar{\nabla}_{Y} Z+\bar{\nabla}_{[X, Y]} Z \\
= & \bar{\nabla}_{Y}\left(\nabla_{X} Z+B(X, Z)\right)-\bar{\nabla}_{X}\left(\nabla_{Y} Z+B(Y, Z)\right)+\nabla_{[X, Y]} Z+B([X, Y], Z) \\
= & \nabla_{Y} \nabla_{X} Z+B\left(Y, \nabla_{X} Z\right)+\nabla_{Y}^{\perp} B(X, Z)-S_{B(X, Z)} Y-\nabla_{X} \nabla_{Y} Z+B\left(X, \nabla_{Y} Z\right) \\
& -\nabla_{X}^{\perp} B(Y, Z)+S_{B(Y, Z)} X+\nabla_{[Y, Z]} Z+B([X, Y], Z) \\
= & R(X, Y) Z+B\left(Y, \nabla_{X} Z\right)+\nabla_{Y}^{\perp} B(X, Z)-S_{B(X, Z)} Y+B\left(X, \nabla_{Y} Z\right) \\
& -\nabla_{X}^{\perp} B(Y, Z)+S_{B(Y, Z)} X+B([X, Y], Z) .
\end{aligned}
$$

Fazendo o produto com $T$ temos

$$
\langle\bar{R}(X, Y) Z, T\rangle=\langle R(X, Y) Z, T\rangle-\left\langle S_{B(X, Z)} Y, T\right\rangle+\left\langle S_{B(Y, Z)} X, T\right\rangle .
$$

Como

$$
\left\langle S_{\eta} E, F\right\rangle=\langle(B(E, F), \eta\rangle
$$

obtemos

$$
\langle\bar{R}(X, Y) Z, T\rangle=\langle R(X, Y) Z, T\rangle+\langle B(X, T), B(Y, Z)\rangle-\langle B(Y, T), B(X, Z)\rangle .
$$

Vamos agora demonstrar a equação de Ricci

$$
\begin{aligned}
\bar{R}(X, Y) \eta= & \bar{\nabla}_{Y} \bar{\nabla}_{X} \eta-\bar{\nabla}_{X} \bar{\nabla}_{Y} \eta+\bar{\nabla}_{[X, Y]} \eta \\
= & \bar{\nabla}_{Y}\left(\nabla_{X}^{\perp} \eta-S_{\eta}(X)\right)-\bar{\nabla}_{X}\left(\nabla_{Y}^{\perp} \eta-S_{\eta}(Y)\right)+\nabla_{[X, Y]}^{\perp} \eta-S_{\eta}([X, Y]) \\
= & \bar{\nabla}_{Y} \nabla_{X}^{\perp} \eta-\bar{\nabla}_{Y} S_{\eta}(X)-\bar{\nabla}_{X} \nabla_{Y}^{\perp} \eta+\bar{\nabla}_{X} S_{\eta}(Y)+\nabla_{[X, Y]}^{\perp} \eta-S_{\eta}([X, Y]) \\
= & \nabla_{Y}^{\perp} \nabla_{X}^{\perp} \eta-S_{\nabla_{X}^{\perp} \eta} Y-\nabla_{Y} S_{\eta}(X)-B\left(Y, S_{\eta}(X)\right)-\nabla_{X}^{\perp} \nabla_{Y}^{\perp} \eta+S_{\nabla_{Y}^{\perp} \eta} X \\
& +\nabla_{X} S_{\eta} Y+B\left(X, S_{\eta}(Y)\right)+\nabla_{[X, Y]}^{\perp} \eta-S_{\eta}([X, Y]) \\
= & R^{\perp}(X, Y) \eta+B\left(X, S_{\eta}(Y)\right)-B\left(Y, S_{\eta}(X)\right)-S_{\nabla_{X}^{\perp} \eta} Y-\nabla_{Y} S_{\eta} X \\
& +S_{\nabla_{Y}^{\perp} \eta} X+\nabla_{X} S_{\eta} Y-S_{\eta}[X, Y] .
\end{aligned}
$$


Fazendo o produto com $\xi$ temos

$$
\begin{aligned}
\langle\bar{R}(X, Y) \eta, \xi\rangle & =\left\langle R^{\perp}(X, Y) \eta, \xi\right\rangle+\left\langle B\left(X, S_{\eta}(Y)\right), \xi\right\rangle-\left\langle B\left(Y, S_{\eta}(X)\right), \xi\right\rangle \\
& =\left\langle R^{\perp}(X, Y) \eta, \xi\right\rangle+\left\langle S_{\xi} X, S_{\eta} Y\right\rangle-\left\langle S_{\xi} Y, S_{\eta} X\right\rangle
\end{aligned}
$$

O resultado segue sabendo que $S_{\xi}$ e $S_{\eta}$ são auto-adjuntas.

Tomando dois vetores $x, y$ ortonormais, decorre da fórmula de Gauss que

$$
K(x, y)-\bar{K}(x, y)=\langle B(x, x), B(y, y)\rangle-|B(x, y)|^{2}
$$

Para o caso de hipersuperfícies $f: M^{n} \rightarrow \bar{M}^{n+1}$, a equação acima admite uma forma mais simples. Sejam $p \in M$ e $\eta \in\left(T_{p} M\right)^{\perp},|\eta|=1$. Seja $\left\{e_{1}, \ldots, e_{n}\right\}$ uma base ortonormal de $T_{p} M$ para a qual $S_{\eta}=S$ é diagonal, isto é, $S\left(e_{i}\right)=\lambda_{i} e_{i}, i=1, \ldots, n$ onde $\lambda_{1}, \ldots, \lambda_{n}$ são os valores próprios de $S$ também chamados de direções principais de $M$ em $p$. Note que $B\left(e_{i}, e_{j}\right)=\lambda_{i j} \eta$, assim

$$
\lambda_{i j}=\left\langle B\left(e_{i}, e_{j}\right), \eta\right\rangle=\lambda_{i}\left\langle e_{i}, e_{j}\right\rangle=\lambda_{i} \delta_{i j}
$$

Portanto $B\left(e_{i}, e_{j}\right)=\lambda_{i} \delta_{i j} \eta$, e segue de (1.92) que

$$
K\left(e_{i}, e_{j}\right)-\bar{K}\left(e_{i}, e_{j}\right)=\lambda_{i} \lambda_{j}
$$

Vamos agora obter a equação de Codazzi. Seja $\mathcal{X}(M)^{\perp}$ o conjunto de campos normais e

$$
\begin{aligned}
B: \mathcal{X}(M) \times \mathcal{X}(M) \times \mathcal{X}(M)^{\perp} & \longrightarrow D(M) \\
(X, Y, \eta) & \longmapsto\langle B(X, Y), \eta\rangle
\end{aligned}
$$

Definamos

$$
\left(\bar{\nabla}_{Z} B\right)(X, Y, \eta)=Z B(X, Y, \eta)-B\left(\nabla_{Z} X, Y, \eta\right)-B\left(X, \nabla_{Z} Y, \eta\right)-B\left(X, Y, \nabla_{Z}^{\perp} \eta\right)
$$

Proposição 1.8 (Equação de Codazzi).

Com a notação acima

$$
\langle\bar{R}(X, Y) Z, \eta\rangle=\left(\bar{\nabla}_{Y} B\right)(X, Y, \eta)-\left(\bar{\nabla}_{X} B\right)(Y, Z, \eta)
$$


Demonstração. Calculemos primeiramente $\left(\bar{\nabla}_{Y} B\right)(X, Y, \eta)$.

$$
\begin{aligned}
\left(\bar{\nabla}_{Y} B\right)(X, Z, \eta)= & Y B(X, Z, \eta)-B\left(\nabla_{Y} X, Z, \eta\right)-B\left(X, \nabla_{Y} Z, \eta\right)-B\left(X, Z, \nabla_{Y}^{\perp} \eta\right) \\
= & Y\langle B(X, Z), \eta\rangle-\left\langle B\left(\nabla_{Y} X\right), \eta\right\rangle-\left\langle B\left(X, \nabla_{Y} Z\right), \eta\right\rangle \\
& -\left\langle B(X, Z), \nabla_{Y}^{\perp} \eta\right\rangle \\
= & \left\langle\nabla_{Y}^{\perp} B(X, Z), \eta\right\rangle-\left\langle B\left(\nabla_{Y} X, Z\right), \eta\right\rangle-\left\langle B\left(X, \nabla_{Y} Z\right), \eta\right\rangle
\end{aligned}
$$

Analogamente

$$
\left(\bar{\nabla}_{X} B\right)(Y, Z, \eta)=\left\langle\nabla_{X}^{\perp} B(Y, Z), \eta\right\rangle-\left\langle B\left(\nabla_{X} Y, Z\right), \eta\right\rangle-\left\langle B\left(Y, \nabla_{X} Z\right), \eta\right\rangle
$$

Por outro lado, na demonstração da Equação de Gauss, deduzimos a seguinte equação

$$
\begin{aligned}
\bar{R}(X, Y) Z= & R(X, Y) Z+B\left(Y, \nabla_{X} Z\right)+\nabla_{Y}^{\perp} B(X, Z)-S_{B(X, Z)} Y+B\left(X, \nabla_{Y} Z\right) \\
& -\nabla_{X}^{\perp} B(Y, Z)+S_{B(Y, Z)} X+B([X, Y], Z) .
\end{aligned}
$$

Fazendo o produto com $\eta$, temos

$$
\begin{aligned}
\langle\bar{R}(X, Y) Z, \eta\rangle= & \left\langle B\left(Y, \nabla_{X} Z\right), \eta\right\rangle+\left\langle\nabla_{Y}^{\perp} B(X, Z), \eta\right\rangle-\left\langle B\left(X, \nabla_{Y} Z\right), \eta\right\rangle \\
& -\left\langle\nabla_{X}^{\perp} B(Y, Z), \eta\right\rangle+\langle B([X, Y], Z), \eta\rangle
\end{aligned}
$$

De (1.93), (1.94) e (1.95), o resultado segue.

Se o espaço ambiente $\bar{M}$ tem curvatura seccional constante, a equação de Codazzi se reduz a

$$
\left(\bar{\nabla}_{X} B\right)(Y, Z, \eta)=\left(\bar{\nabla}_{Y} B\right)(X, Z, \eta)
$$

Se, além disto, a codimensão da imersão é igual a 1 , podemos concluir que $\nabla_{X}^{\perp} \eta=0$, de onde,

$$
\begin{aligned}
\left(\bar{\nabla}_{X} B\right)(Y, Z, \eta) & =X\langle B(Y, Z), \eta\rangle-\left\langle B\left(\nabla_{X}^{Y}, Z\right), \eta\right\rangle-\left\langle B\left(Y, \nabla_{Z} X\right), \eta\right\rangle \\
& =X\left\langle S_{\eta}(Y), Z\right\rangle-\left\langle S_{\eta}\left(\nabla_{X} Y\right), Z\right\rangle-\left\langle S_{\eta}(Y), \nabla_{X} Z\right\rangle \\
& =\left\langle\nabla_{X} S_{\eta}(Y), Z\right\rangle-\left\langle S_{\eta}\left(\nabla_{X} Y\right), Z\right\rangle
\end{aligned}
$$

Analogamente,

$$
\left(\bar{\nabla}_{Y} B\right)(X, Z, \eta)=\left\langle\nabla_{Y} S_{\eta}(X), Z\right\rangle-\left\langle S_{\eta}\left(\nabla_{Y} X\right), Z\right\rangle
$$

$\operatorname{De}\left(\bar{\nabla}_{X} B\right)(Y, Z, \eta)=\left(\bar{\nabla}_{Y} B\right)(X, Z, \eta)$, obtemos

$$
\left\langle\nabla_{X} S_{\eta}(Y), Z\right\rangle-\left\langle S_{\eta}\left(\nabla_{X} Y\right), Z\right\rangle=\left\langle\nabla_{Y} S_{\eta}(X), Z\right\rangle-\left\langle S_{\eta}\left(\nabla_{Y} X\right), Z\right\rangle
$$


Como $Z$ é arbitrário

$$
\nabla_{X} S_{\eta}(Y)-\nabla_{Y} S_{\eta}(X)=S_{\eta}([X, Y])
$$

Como vamos lidar com casos onde a codimensão é igual a 1, vamos ver como ficam as equações fundamentais em tais casos.

\section{Proposição 1.9 .}

Sejam $f: M \rightarrow \bar{M}$ uma imersão isométrica de codimensão igual a 1; X, Y, Z campos tangentes a $M$ e $N$ um campo normal unitário a $M$, então as seguintes equações se verificam:

(a) Equação de Gauss

$$
R(X, Y) Z-\bar{R}(X, Y) Z=\langle S X, Z\rangle S Y-\langle S Y, Z\rangle S X
$$

(b) Equação de Codazzi

$$
\bar{R}(X, Y) N=\nabla_{X} S(Y)-\nabla_{Y} S(X)-S([X, Y])
$$

Demonstração. Como passo intermediário na demonstração da equação de Ricci, deduzimos a seguinte equação

$$
\bar{R}(X, Y) \eta=\bar{\nabla}_{Y} \nabla_{X}^{\perp} \eta-\bar{\nabla}_{Y} S_{\eta}(X)-\bar{\nabla}_{X} \nabla_{Y}^{\perp} \eta+\bar{\nabla}_{X} S_{\eta}(Y)+\nabla_{[X, Y]}^{\perp} \eta-S_{\eta}([X, Y]) .
$$

Como já observamos, se a codimensão da imersão é igual a $1, \nabla_{X}^{\perp} \eta=0$. Assim

$$
\bar{R}(X, Y) \eta=\bar{\nabla}_{X} S_{\eta}(Y)-\bar{\nabla}_{Y} S_{\eta}(X)-S_{\eta}([X, Y])
$$

Note que

$$
\begin{aligned}
\left\langle\bar{\nabla}_{X} S Y-\bar{\nabla}_{Y} S X, N\right\rangle & =\left\langle\bar{\nabla}_{X} S Y, N\right\rangle-\left\langle\bar{\nabla}_{Y} S X, N\right\rangle \\
& =X\langle S Y, N\rangle-\left\langle S Y, \bar{\nabla}_{X} N\right\rangle-Y\langle S X, N\rangle+\left\langle S X, \bar{\nabla}_{Y} N\right\rangle \\
& =-\langle S Y,-S X\rangle+\langle S X,-S Y\rangle \\
& =0 .
\end{aligned}
$$

Isto é, o campo $\bar{\nabla}_{X} S Y-\bar{\nabla}_{Y} S X$ é um campo tangente a $M$. Logo, podemos trocar $\bar{\nabla}_{X} S Y-\bar{\nabla}_{Y} S X$ por $\nabla_{X} S Y-\nabla_{Y} S X$ na equação (1.96), obtendo

$$
\bar{R}(X, Y) \eta=\nabla_{X} S_{\eta}(Y)-\nabla_{Y} S_{\eta}(X)-S_{\eta}([X, Y])
$$


A equação de Ricci, no caso de codimensão igual a 1, reduz-se a uma identidade trivial. Vejamos como fica a equação de Gauss. Sejam $X, Y, Z, T \in \mathcal{X}(\bar{M})$ e $N \in \mathcal{X}(M)^{\perp}$ um campo normal unitário. Se a codimensão for igual a 1 , temos que $B(X, T)=\lambda N$, de onde

$$
\lambda=\langle B(X, T), N\rangle=\langle S X, T\rangle .
$$

Utilizando esta observação, podemos reescrever a equação de Gauss como

$$
\langle R(X, Y) Z-\bar{R}(X, Y) Z, T\rangle=\langle\langle S X, Z\rangle N,\langle S Y, T\rangle N\rangle-\langle\langle S Y, Z\rangle N,\langle S X, T\rangle N\rangle,
$$

isto é,

$$
\langle R(X, Y) Z-\bar{R}(X, Y) Z, T\rangle=\langle\langle S Y, Z\rangle S X-\langle S X, Z\rangle S Y, T\rangle
$$

A equação (1.97) é válida para qualquer $T \in \bar{M}$, em particular substituindo $T$ por $N$, obtemos

$$
\langle R(X, Y) Z-\bar{R}(X, Y) Z, N\rangle=0
$$

ou seja, $R(X, Y) Z-\bar{R}(X, Y) Z$ é um campo tangente a $M$. Logo, de (1.97), podemos concluir que

$$
R(X, Y) Z-\bar{R}(X, Y) Z=\langle S X, Z\rangle S Y-\langle S Y, Z\rangle S X
$$

\subsection{O princípio do Máximo}

Nesta seção recordaremos o clássico Princípio do Máximo (PM) de H. Hopf para equações elípticas de segunda ordem. Não estamos interessados em EDPs, mas sim em suas aplicações a superfícies.

Estamos interessados em equações em derivadas parciais da forma

$$
\sum_{i, j=1}^{n} a_{i j} u_{x_{i} x_{j}}+\sum_{i=1}^{n} b_{i} u_{x_{i}}+c u=d,
$$

onde $a_{i j}, b_{j}, c$ e $d$ são funções reais de classe $C^{\infty}$ que dependem somente de $\left(x_{1}, \ldots, x_{n}\right) \in \Omega$, sendo $\Omega$ um domínio de $\mathbb{R}^{n}$. Além disso, a matriz formada pelos $a_{i j}$, ou seja $A=$ $\left(a_{i j}\right)_{i, j=1, \ldots, n}$, é uma matriz simétrica e $u$ é uma função diferenciável em $\Omega$. 
Podemos representar a equação (1.98) por

$$
L u=d
$$

onde $L$ é o operador definido por

$$
L \equiv \sum_{i, j=1}^{n} a_{i j} \frac{\partial^{2}}{\partial x_{i} \partial x_{j}}+\sum_{i=1}^{n} b_{i} \frac{\partial}{\partial x_{i}}+c .
$$

Ao operador $L$ associaremos a forma quadrática $Q: \mathbb{R}^{n} \rightarrow \mathbb{R}$ dada pela matriz $A$, isto é,

$$
Q(x)=Q\left(x_{1}, \ldots, x_{n}\right)=\sum_{i, j=1}^{n} a_{i j} x_{i} x_{j}
$$

O seguinte passo é identificar os tipos de operadores $L$, dados por (1.99), dependendo da forma quadrática associada

Definição 1.5. Diremos que um operador $L$ do tipo dado em (1.99) é

- Elíptico se os autovalores da matriz $A$ são todos positivos.

- Uniformemente elíptico se os autovalores da matriz A possuem uma cota inferior constante positiva.

- Hiperbólico se os autovalores da matriz A são todos não nulos, mas existem autovalores de sinais opostos.

- Parabólico se algum autovalor se anula.

Os métodos do princípio do máximo estão baseados na seguinte observação: dada uma função $u \in C^{2}(\Omega)$ tal que alcança um máximo em um ponto $x_{0} \in \Omega$, então $\operatorname{grad}_{0} u\left(x_{0}\right)=0$ e $\operatorname{Hess}_{0} u\left(x_{0}\right) \leq 0$, onde $\operatorname{grad}_{0}$ e Hess 0 denotam o gradiente e o Hessiano de $u$ em um ponto $x_{0}$.

O PM fraco nos diz que, se $L u$ tem sinal, então o máximo ou o mínimo (dependendo do sinal de $L u$ ) de $u$ no fecho de $\Omega$ alcança-se em algum ponto da fronteira de $\Omega$, ainda que também possa alcançar-se em algum outro ponto do interior.

Por sua vez, o PM forte nos diz que, sob certas condições sobre o operador $L$, se $L u$ tem sinal e o máximo ou o mínimo (dependendo do sinal de $L u$ ) alcança-se em um ponto interior, então $u$ deve ser constante. 


\section{Teorema 1.1.}

Seja $L$ um operador uniformemente elíptico em um dominio $\Omega \subset \mathbb{R}^{n}$. Suponhamos $L u \geq 0$ para uma função $u \in C^{2}(\Omega)$. Então

- se c $\equiv 0$ e u alcança seu máximo em $\Omega$, u é constante.

- se c $\leq 0, u$ alcança seu máximo em $\Omega$ e esse máximo é não negativo, u é constante.

Teorema 1.2.

Seja $L$ um operador uniformemente elíptico em um dominio $\Omega \subset \mathbb{R}^{n}$, com fronteira $\partial \Omega$ duas vezes diferenciável. Seja $x_{0} \in \partial \Omega$ tal que

1. $u$ é de classe $C^{1}$ em $x_{0}$.

2. $u\left(x_{0}\right) \geq u_{x}, \quad \forall x \in \Omega$.

3. $\frac{\partial u}{\partial \eta}\left(x_{0}\right)=0$, onde $\eta$ é o normal interior de $\partial \Omega$.

\section{Então}

- se $c \equiv 0, u$ é constante.

- se c $\leq 0$ e $u\left(x_{0}\right) \geq 0$, u é constante. 


\section{Espaços produto}

Neste capítulo, veremos a definição de espaços produto, como se comporta o tensor curvatura em tais espaços e particularizaremos as equações de Gauss e Codazzi. Veremos também as equações de compatibilidade que uma superfície imersa nestes espaços deve satisfazer.

\section{$2.1 \quad$ Aspectos gerais}

Ao longo deste capítulo, trataremos com uma variedade 3 -dimensional $\mathbb{M}^{2} \times \mathbb{R}$ dada pelo produto de uma superfície Riemanniana $\mathbb{M}^{2}$, a que chamaremos de base, e a reta real $\mathbb{R}$, chamada fibra.

Um caso especial deste tipo de variedade é quando consideramos que sua base seja uma superfície simplesmente conexa com curvatura constante, isto é, uma das formas espaciais bidimensionais. Quando a curvatura da base é constante e igual a zero, o espaço produto que obtemos é o espaço euclidiano tridimensional; portanto, omitiremos este caso. Quando dissermos que a curvatura da base é constante, entenderemos que essa constante é diferente de zero. Além disso, podemos supor que a curvatura é \pm 1 . Com efeito, se denotarmos por $g_{c}$ a métrica de curvatura constante $c$, então a métrica $|c| g_{c}$ tem curvatura $\frac{c}{|c|}$. Assim, teremos que quando a curvatura da base é 1 , nossa base é $\mathbb{S}^{2}$, e quando é -1 a base é $\mathbb{H}^{2}$. Para agrupar, escreveremos $\mathbb{M}^{2}(\varepsilon), \varepsilon=1,-1$, sendo, $\mathbb{M}^{2}(1)=\mathbb{S}^{2}$ e $\mathbb{M}^{2}(-1)=\mathbb{H}^{2}$.

Seja $\mathbb{M}^{2}$ uma superfície Riemanniana, cuja métrica denotaremos por $g_{\kappa}$, onde $\kappa$ é a curvatura de Gauss de $g_{\kappa}$, e consideraremos a variedade produto $\mathbb{M}^{2} \times \mathbb{R}$. Sejam $\pi$ e $\sigma$ as projeções sobre a base, $\mathbb{M}^{2}$, e a fibra $\mathbb{R}$, respectivamente. Então a métrica em $\mathbb{M}^{2} \times \mathbb{R}$ é dada por

$$
\langle,\rangle=\pi^{*}\left(g_{\kappa}\right)+\sigma^{*}\left(d t^{2}\right)
$$

onde $d t^{2}$ é a métrica padrão em $\mathbb{R}$.

Chamaremos seção horizontal, $\mathbb{M}^{2} \times\{t\}=\sigma^{-1}(t)$, a pré-imagem por $\sigma$ de um 
ponto $t$ da fibra, e seção vertical, $\{p\} \times \mathbb{R}=\pi^{-1}(p)$, a pré-imagem por $\pi$ de um ponto $p$ da base.

Além disso, é claro que a métrica produto $\langle$,$\rangle verifica:$

- Para cada $t \in \mathbb{R}$, a aplicação $\left.\pi\right|_{\mathbb{M}^{2} \times\{t\}}$ é um isometria.

- Para cada $p \in \mathbb{M}^{2}$, a aplicação $\left.\sigma\right|_{\{p\} \times \mathbb{R}}$ é uma isometria.

- Para cada $(p, t) \in \mathbb{M}^{2} \times \mathbb{R}$, a seção horizontal, $\mathbb{M}^{2} \times\{t\}$, e a vertical, $\{p\} \times \mathbb{R}$ são ortogonais em $(p, t)$.

Por comodidade omitiremos as projeções, sempre e quando não haja confusão utilizando essa notação. Por exemplo, adiante escreveremos

$$
\langle,\rangle=g_{\kappa}+d t^{2}
$$

É claro que a geometria do espaço produto depende fortemente da geometria tanto de sua base como de sua fibra. Para o caso em que a fibra é a reta real, nosso caso, dita dependência é maior da geometria da base que da fibra. Para ver como dependem uma da outra, uma ferramenta básica é a noção de campos horizontais e verticais, isto é, a projeção na base e na fibra, respectivamente, de um campo na variedade.

Dado $X \in \mathcal{X}\left(\mathbb{M}^{2} \times \mathbb{R}\right)$ um campo de vetores, temos que

$$
X(p, t)=\left(X^{h}(p), X^{v}(t)\right) \in T_{(p, t)}\left(\mathbb{M}^{2} \times \mathbb{R}\right) \equiv T_{p} \mathbb{M}^{2} \times T_{t} \mathbb{R}
$$

onde $X^{h}=d \pi(X) \in \mathcal{X}\left(\mathbb{M}^{2}\right)$ e $X^{v}=d \sigma(X) \in \mathcal{X}(\mathbb{R})$. Então, dizemos que $X \in \mathcal{X}\left(\mathbb{M}^{2} \times \mathbb{R}\right)$ é um campo horizontal (respectivamente campo vertical) se $X^{v} \equiv 0$ (respectivamente $\left.X^{h} \equiv 0\right)$.

Se $X$ é um campo horizontal, então $X \in \mathcal{X}\left(\mathbb{M}^{2}\right) \subset \mathcal{X}\left(\mathbb{M}^{2} \times \mathbb{R}\right)$, e seguiremos denotando por $X$ visto como campo em $\mathbb{M}^{2}$, sempre e quando não houver confusão com a notação empregada. Analogamente, se $Y$ é um campo vertical, $Y \in \mathcal{X}(\mathbb{R}) \subset \mathcal{X}\left(\mathbb{M}^{2} \times \mathbb{R}\right)$, e seguiremos denotando por $Y$ visto como campo em $\mathbb{R}$.

Denotaremos por $\bar{\nabla}, \nabla^{\mathbb{M}^{2}}$ e $\nabla^{\mathbb{R}}$, as conexões de Levi-Civita associadas a $\langle\rangle,, g_{\kappa}$ e $d t^{2}$, respectivamente.

Para estudar como atua a conexão do ambiente somente é necessário saber como atua sobre campos horizontais e verticais. Isto é o que nos diz o seguinte resultado (ver [6], Proposição 56, pág. 89). 


\section{Proposição 2.1.}

Sejam $X, Y \in \mathcal{X}\left(\mathbb{M}^{2}\right)$ e $V, W \in \mathcal{X}(\mathbb{R})$, então

(1) $\bar{\nabla}_{X} Y$ é um campo horizontal e $\left(\bar{\nabla}_{X} Y\right)^{h}=\nabla_{X}^{\mathbb{M}^{2}} Y \in \mathcal{X}\left(\mathbb{M}^{2}\right)$.

(2) $\bar{\nabla}_{V} W$ é um campo vertical e $\left(\bar{\nabla}_{V} W\right)^{v}=\nabla_{V}^{\mathbb{R}} W \in \mathcal{X}(\mathbb{R})$.

(3) $\bar{\nabla}_{V} X=\bar{\nabla}_{X} V=0$.

A partir deste primeiro resultado, que se obtém de uma forma direta usando as fórmulas de Koszul, poderemos estudar tanto as geodésicas, como a completude do espaço produto (veja [6], Corolário 57, pág. 89).

Corolário 2.1.(1) Uma curva $\gamma(s)=(\alpha(s), \beta(s)) \subset \mathbb{M}^{2} \times \mathbb{R}$ é uma geodésica se, e somente se, suas projeções $\alpha \subset \mathbb{M}^{2}$ e $\beta \subset \mathbb{R}$ são ambas geodésicas.

(2) $\mathbb{M}^{2} \times \mathbb{R}$ é completa se, e somente se, $\mathbb{M}^{2}$ é completa (notemos que nossa fibra é a reta real com a métrica usual, que é completa).

Dado um campo horizontal $X \in \mathcal{X}\left(\mathbb{M}^{2}\right)$, temos

$$
\langle\bar{\nabla} \sigma, X\rangle=d \sigma(X)=0
$$

onde $\bar{\nabla} \sigma$ denota o gradiente de $\sigma$ com respeito a $\langle$,$\rangle . Portanto \bar{\nabla} \sigma \in \mathcal{X}(\mathbb{R})$, que denotaremos no que se segue por $\frac{\partial}{\partial t}$, é uma base de $\mathcal{X}(\mathbb{R})$. Além disso, $\left\langle\frac{\partial}{\partial t}, \frac{\partial}{\partial t}\right\rangle=1$.

Logo, dado um campo $X \in \mathcal{X}\left(\mathbb{M}^{2} \times \mathbb{R}\right)$, temos

$$
X^{h}=X-\left\langle X, \frac{\partial}{\partial t}\right\rangle \frac{\partial}{\partial t}, \quad X^{v}=\left\langle X, \frac{\partial}{\partial t}\right\rangle \frac{\partial}{\partial t} .
$$

Como sempre, escreveremos $\partial_{t}$ no lugar de $\frac{\partial}{\partial t}$. Observa-se que $\partial_{t}$ é um campo paralelo, o que segue da Proposição 2.1 e de que

$$
\left\langle\bar{\nabla}_{\partial_{t}} \partial_{t}, \partial_{t}\right\rangle=\frac{1}{2} \partial_{t}\left\langle\partial_{t}, \partial_{t}\right\rangle=0
$$

De fato, se $X \in\left(\mathbb{M}^{2} \times \mathbb{R}\right)$, temos

$$
\bar{\nabla}_{X} \partial_{t}=\bar{\nabla}_{X^{h}} \partial_{t}+\left\langle X, \partial_{t}\right\rangle \bar{\nabla}_{\partial_{t}} \partial_{t}=0
$$

logo $\partial_{t}$ é um campo paralelo.

Agora veremos como se relacionam os tensores de curvatura $\bar{R},{ }^{\mathbb{M}^{2}} R$ e ${ }^{\mathbb{R}} R$ de $\mathbb{M}^{2} \times \mathbb{R}, \mathbb{M}^{2}$ e $\mathbb{R}$ respectivamente (veja [6], Corolário 58, pág. 89). 


\section{Corolário 2.2.}

Sejam $X, Y, Z \in \mathcal{X}\left(\mathbb{M}^{2}\right)$ e $U, V, W \in \mathcal{X}(\mathbb{R})$. Então

(1) $\bar{R}(X, Y) Z$ é um campo horizontal e $(\bar{R}(X, Y) Z)^{h}={ }^{\mathbb{M}^{2}} R(X, Y) Z \in \mathcal{X}\left(\mathbb{M}^{2}\right)$.

(2) $\bar{R}(U, V) W$ é um campo vertical e $(\bar{R}(U, V) W)^{v}={ }^{\mathbb{R}} R(U, V) W \in \mathcal{X}(\mathbb{R})$.

(3) $\bar{R}$ é zero para qualquer outra escolha de $X, \ldots, W$.

Deste último Corolário, e tendo em conta que ${ }^{\mathbb{R}} R \equiv 0$, temos

\section{Corolário 2.3.}

Sejam $X, Y, Z \in \mathcal{X}\left(\mathbb{M}^{2} \times \mathbb{R}\right)$ e denotemos por $X^{h}, Y^{h}, Z^{h} \in \mathcal{X}\left(\mathbb{M}^{2}\right)$ as suas projeções horizontais. Então

$$
\bar{R}(X, Y) Z={ }^{\mathbb{M}^{2}} R\left(X^{h}, Y^{h}\right) Z^{h} .
$$

Chamaremos de slices aos conjuntos da forma $\mathbb{M}^{2}(\varepsilon) \times\left\{t_{0}\right\}$, para algum $t_{0} \in \mathbb{R}$ fixo.

\subsection{Equações de compatibilidade}

Ao contrário do que ocorre em formas espaciais, as equações de Gauss e Codazzi não descrevem completamente o comportamento da superfície. Assim, o que faremos será obter uma série de equações necessárias, entre elas a de Gauss e Codazzi, que uma superfície imersa no espaço produto tem de satisfazer .

Para os casos particulares em que a base é $\mathbb{H}^{2}$ ou $\mathbb{S}^{2}$, tais equações são de fato as equações de integrabilidade da superfície.

Vamos considerar o espaço homogêneo $\mathbb{S}^{2} \times \mathbb{R}$ como a hipersuperfície do espaço euclidiano tetradimensional usual $\mathbb{R}^{4}$ dada por

$$
\mathbb{S}^{2} \times \mathbb{R}=\left\{\left(x_{1}, x_{2}, x_{3}, x_{4}\right) \in \mathbb{R}^{4}: x_{1}^{2}+x_{2}^{2}+x_{3}^{2}=1\right\}
$$

Vamos também denotar $\mathbb{H}^{2} \times \mathbb{R}$ como a subvariedade Riemanniana do espaço de Lorentz $\mathbb{L}^{4}$, com métrica induzida $-x_{1}^{2}+x_{2}^{2}+x_{3}^{2}+x_{4}^{2}$, dada por

$$
\mathbb{H}^{2} \times \mathbb{R}=\left\{\left(x_{1}, x_{2}, x_{3}, x_{4}\right) \in \mathbb{R}^{4}:-x_{1}^{2}+x_{2}^{2}+x_{3}^{2}=-1, x_{1}>0\right\}
$$


Sejam $\Sigma$ uma superfície orientável e $\psi: \Sigma \rightarrow \mathbb{M}^{2} \times \mathbb{R}$ uma imersão. Então, consideraremos sobre $\psi$ a métrica induzida (Primeira Forma Fundamental), $\left.\langle\rangle\right|_{,\Sigma}$, de $\mathbb{M}^{2} \times \mathbb{R}$, a qual denotaremos por $I$. Sejam $\nabla$ e $R$ a conexão e o tensor de curvatura de $\psi$, respectivamente, e $S$ o endomorfismo de Weingarten de $S$ associado ao normal unitário $N$ da superfície, isto é, $S X=-\bar{\nabla}_{X} N, X \in \mathcal{X}(\psi)$. Então $I I(X, Y)=\langle S X, Y\rangle$ é a Segunda Forma Fundamental da superfície. Além disso, denotemos por:

(1) $T$ a projeção do vetor vertical $\partial_{t}$ sobre o espaço tangente de $\Sigma$.

(2) $\nu=\left\langle N, \partial_{t}\right\rangle$, a componente normal de $N$. A $\nu$ chamaremos função ângulo da superfície.

(3) $h$ a função altura em $\Sigma$, isto é, a quarta coordenada da imersão $\psi$.

Observamos primeiramente que $T$ é igual ao gradiente da função altura $h$. De fato, como $T \in \mathcal{X}(\Sigma)$, temos

$$
T=a \partial_{u}+b \partial_{v}
$$

para algumas funções reais $a, b$. Agora, escreveremos o campo $\partial_{t}$ em sua parte tangente e normal, isto é,

$$
\partial_{t}=T+\nu N
$$

Fazendo o produto de (2.1), com $\partial_{u}$ e $\partial_{v}$, obtemos

$$
\begin{aligned}
& h_{u}=\left\langle\partial_{t}, \partial_{u}\right\rangle=a E+b F, \\
& h_{v}=\left\langle\partial_{t}, \partial_{v}\right\rangle=a F+b G,
\end{aligned}
$$

de onde segue

$$
a=\frac{G h_{u}-F h_{v}}{E G-F^{2}} \quad \text { e } \quad b=\frac{-F h_{u}+E h_{v}}{E G-F^{2}} .
$$

Logo

$$
T=\frac{G h_{u}-F h_{v}}{E G-F^{2}} \partial_{u}+\frac{-F h_{u}+E h_{v}}{E G-F^{2}} \partial_{v}=\operatorname{grad} h .
$$

\section{Proposição 2.2.}

Sejam $\Sigma$ uma superfície orientável e $\psi: \Sigma \rightarrow \mathbb{M}^{2}(\varepsilon) \times \mathbb{R}$ uma imersão com campo normal unitário $N$ e operador forma associado $S$. Denotaremos por h a função altura em $\Sigma$, por 
$\nu$ a quarta coordenada do normal unitário $N$ e por $T$ a projeção do vetor vertical $\frac{\partial}{\partial_{t}}$ sobre o espaço tangente de $\Sigma$. Então as seguintes equações são satisfeitas

$$
\begin{array}{lr}
K(I)=K+\varepsilon \nu^{2}, & \text { Gauss } \\
\nabla_{X} S Y-\nabla_{Y} S X-S[X, Y]=\varepsilon \nu(\langle Y, T\rangle X-\langle X, T\rangle Y), & \text { Codazzi } \\
\nabla_{X} T=\nu S X, & \\
d \nu(X)=-\langle S X, T\rangle, & \\
\|T\|^{2}+\nu^{2}=1 . &
\end{array}
$$

Demonstração. De (2.1)

$$
1=\left\langle\partial_{t}, \partial_{t}\right\rangle=\langle T+\nu N, T+\nu N\rangle=\|T\|^{2}+\nu^{2}
$$

e está demonstrado (2.6). Para demonstrar a terceira e quarta equações, lembre que $\partial_{t}$ é um campo paralelo, logo $\bar{\nabla}_{X} \partial_{t}=0$. Assim, de (2.1), obtemos

$$
0=\bar{\nabla}_{X} T+(d \nu(X)) N+\nu \bar{\nabla}_{X} N
$$

Podemos escrever

$$
\bar{\nabla}_{X} T=\left(\bar{\nabla}_{X} T\right)^{t}+c N
$$

Fazendo o produto com $N$ e notando que $\left\langle\left(\bar{\nabla}_{X} T\right)^{t}, N\right\rangle=0$, concluímos que $\left\langle\bar{\nabla}_{X} T, N\right\rangle=c$. Como $\langle T, N\rangle=0$, resulta que

$$
\left\langle\bar{\nabla}_{X} T, N\right\rangle+\left\langle T, \bar{\nabla}_{X} N\right\rangle=0
$$

$\log 0$

$$
\left\langle\bar{\nabla}_{X} T, N\right\rangle=-\left\langle T, \bar{\nabla}_{X} N\right\rangle=\langle T, S X\rangle
$$

Segue de (2.7)

$$
0=\nabla_{X} T+\langle S X, T\rangle N+(d \nu(X)) N-\nu S X
$$

Tomando a parte tangente e a parte normal nesta igualdade, obtemos

$$
\nabla_{X} T=\nu S X \quad \text { e } \quad d \nu(X)=-\langle S X, T\rangle .
$$


Dados $X, Y, Z \in \mathcal{X}(\psi)$, as equações de Gauss e Codazzi de uma superfície em um espaço tridimensional são dadas, respectivamente, por

$$
\begin{aligned}
R(X, Y) Z-\bar{R}(X, Y) Z & =\langle S X, Z\rangle S Y-\langle S Y, Z\rangle S X \\
\bar{R}(X, Y) N & =\nabla_{X} S Y-\nabla_{Y} S X-S[X, Y]
\end{aligned}
$$

Logo, do Corolário (2.3), e usando que o tensor de curvatura de uma superfície sempre se pode expressar como

$$
\mathbb{M}^{2} R\left(X^{h}, Y^{h}\right) Z^{h}=\varepsilon\left(\left\langle X^{h}, Z^{h}\right\rangle Y^{h}-\left\langle Y^{h}, Z^{h}\right\rangle X^{h}\right)
$$

onde $\varepsilon$ é a curvatura de Gauss de $\mathbb{M}^{2}$ no ponto em que estamos trabalhando, podemos escrever,

$$
X^{h}=X-c \partial_{t}
$$

para alguma função real $c$. Efetuando o produto, na equação acima por $\partial_{t}$, e observando que $\left\langle X^{h}, \partial_{t}\right\rangle=0$, obtemos que $c=\left\langle X, \partial_{t}\right\rangle$, assim

$$
X^{h}=X-\left\langle X, \partial_{t}\right\rangle \partial_{t}
$$

Portanto para quaisquer $X, Y, Z \in \mathcal{X}\left(\mathbb{M}^{2} \times \mathbb{R}\right)$

$$
\begin{aligned}
\bar{R}(X, Y) Z= & { }^{\mathbb{M}^{2}} R\left(X^{h}, Y^{h}\right) Z^{h} \\
= & \varepsilon\left(\left\langle X^{h}, Z^{h}\right\rangle Y^{h}-\left\langle Y^{h}, Z^{h}\right\rangle X^{h}\right) \\
= & \left(\left(\langle X, Z\rangle-\left\langle X, \partial_{t}\right\rangle\left\langle Z, \partial_{t}\right\rangle\right)\left(Y-\left\langle Y, \partial_{t}\right\rangle \partial_{t}\right)\right. \\
& \left.-\left(\langle Y, Z\rangle-\left\langle Y, \partial_{t}\right\rangle\left\langle Z, \partial_{t}\right\rangle\right)\left(X-\left\langle X, \partial_{t}\right\rangle \partial_{t}\right)\right) \\
= & \varepsilon\left(\langle X, Z\rangle Y-\langle Y, Z\rangle X-\left\langle X, \partial_{t}\right\rangle\left\langle Z, \partial_{t}\right\rangle Y+\left\langle Y, \partial_{t}\right\rangle\left\langle Z, \partial_{t}\right\rangle X\right. \\
& \left.+\langle Y, Z\rangle\left\langle X, \partial_{t}\right\rangle-\langle X, Z\rangle\left\langle Y, \partial_{t}\right\rangle \partial_{t}\right),
\end{aligned}
$$

isto é,

$$
\begin{aligned}
\bar{R}(X, Y) Z= & \varepsilon\left(\langle X, Z\rangle Y-\langle Y, Z\rangle X-\left\langle X, \partial_{t}\right\rangle\left\langle Z, \partial_{t}\right\rangle Y+\left\langle Y, \partial_{t}\right\rangle\left\langle Z, \partial_{t}\right\rangle X\right. \\
& \left.+\langle Y, Z\rangle\left\langle X, \partial_{t}\right\rangle-\langle X, Z\rangle\left\langle Y, \partial_{t}\right\rangle \partial_{t}\right) .
\end{aligned}
$$

Assim, tomando $Z=N$ e $X, Y \in \mathcal{X}(\psi)$ em (2.10)

$$
\bar{R}(X, Y) N=\varepsilon \nu(\langle Y, T\rangle X-\langle X, T\rangle Y)
$$


e temos, por (2.9), que a equação de Codazzi (2.3) se verifica.

Para obter a equação de Gauss o que faremos é multiplicar por um campo $W$ a equação (2.8). Assim, de (2.10)

$$
\begin{aligned}
\langle\bar{R}(X, Y) Z, W\rangle= & \varepsilon(\langle X, Z\rangle\langle Y, W\rangle-\langle Y, Z\rangle\langle X, W\rangle \\
& -\left\langle X, \partial_{t}\right\rangle\left\langle Z, \partial_{t}\right\rangle\langle Y, W\rangle+\left\langle Y, \partial_{t}\right\rangle\left\langle Z, \partial_{t}\right\rangle\langle X, W\rangle \\
& \left.+\left(\langle Y, Z\rangle\left\langle X, \partial_{t}\right\rangle-\langle X, Z\rangle\left\langle Y, \partial_{t}\right\rangle\right)\left\langle\partial_{t}, W\right\rangle\right)
\end{aligned}
$$

e tomando $X, Y \in \mathcal{X}(\psi)$ uma base ortonormal e fazendo $Z=X, W=Y,(2.11)$ fica

$$
\begin{aligned}
\langle\bar{R}(X, Y) X, Y\rangle & =\varepsilon\left(1-\left\langle X, \partial_{t}\right\rangle^{2}-\left\langle Y, \partial_{t}\right\rangle^{2}\right) \\
& =\varepsilon\left(1-\langle X, T\rangle-\langle Y, T\rangle^{2}\right) \\
& =\varepsilon\left(1-\|T\|^{2}\right) \\
& =\varepsilon \nu^{2},
\end{aligned}
$$

isto é,

$$
\langle\bar{R}(X, Y) X, Y\rangle=\varepsilon \nu^{2}
$$

Só falta notar que, dados $X, Y \in \mathcal{X}(\psi)$ linearmente independentes, temos

$$
\langle R(X, Y) X, Y\rangle=K(I)
$$

e

$$
K=\operatorname{det} S=\langle S X, X\rangle\langle S Y, Y\rangle-\langle S Y, X\rangle\langle S Y, X\rangle
$$

Em consequência, substituindo (2.12) e (2.13) em (2.9), obtemos (2.2).

Vamos ver agora como ficam as equações (2.2) - (2.6) quando z é um parâmetro conforme para a segunda forma fundamental.

\section{Lema 2.1.}

Seja $\Sigma$ um superfície orientável e $\psi: \Sigma \rightarrow \mathbb{M}^{2}(\varepsilon) \times \mathbb{R}$ uma imersão com curvatura extrínseca positiva, $K>0$. Assim, as equações de compatibilidade para a imersão $\psi$ podem ser 
escritas em um parâmetro conforme para a segunda forma fundamental como

$$
\begin{array}{lr}
K(I)=K+\varepsilon \nu^{2}, & \text { Gauss } \\
\frac{\rho_{\bar{z}}}{\rho}+\left(\Gamma_{12}^{1}-\Gamma_{22}^{2}\right)=\varepsilon \alpha \frac{\nu}{\rho}, & \text { Codazzi } \\
h_{z z}=\Gamma_{11}^{1} h_{z}+\Gamma_{11}^{2} h_{\bar{z}}, & \\
h_{z \bar{z}}=\Gamma_{12}^{1} h_{z}+\Gamma_{12}^{2} h_{\bar{z}}+\nu \rho, & \\
\nu_{\bar{z}}=\frac{\alpha K}{\rho}, & \\
\frac{1}{D}\left(\alpha h_{z}+\bar{\alpha} h_{\bar{z}}\right)+\nu^{2}=1 . &
\end{array}
$$

Demonstração. Como a curvatura extrínseca $K$ é positiva, sua segunda forma, $I I$, é definida. Escolhamos $N$ tal que $I I$ é positiva definida. Como vamos considerar $z$ um parâmetro complexo conforme para a métrica Riemanniana $I I$, podemos escrever

$$
\begin{aligned}
I & =\langle d \psi, d \psi\rangle=L d z^{2}+2 M|d z|^{2}+\bar{L} d \bar{z}^{2} \\
I I & =\langle d \psi,-d N\rangle=2 \rho|d z|^{2}
\end{aligned}
$$

Primeiramente, observe que a curvatura extrínseca é dada por

$$
K=-\frac{\rho^{2}}{D}
$$

onde $D=|L|^{2}-M^{2}<0$.

Podemos tomar

$$
T=\frac{1}{D}\left(\alpha \partial_{z}+\bar{\alpha} \partial_{\bar{z}}\right)
$$

onde

$$
\alpha=\bar{L} h_{z}-M h_{\bar{z}}
$$

Agora, tomando $X=\partial_{\bar{z}}$ e $Y=\partial_{z}$ em (2.3), temos

$$
\nabla_{\partial_{\bar{z}}} S \partial_{z}-\nabla_{\partial_{z}} S \partial_{\bar{z}}-S\left[\partial_{\bar{z}}, \partial_{z}\right]=\varepsilon \nu\left(\left\langle\partial_{z}, T\right\rangle \partial_{\bar{z}}-\left\langle\partial_{\bar{z}}, T\right\rangle \partial_{z}\right) .
$$


Note que $\left[\partial_{\bar{z}}, \partial_{z}\right]=0 \mathrm{e}$

$$
\begin{aligned}
\left\langle\partial_{z}, T\right\rangle & =\frac{\left(\bar{L} h_{z}-M h_{\bar{z}}\right)\left\langle\partial_{z}, \partial_{z}\right\rangle+\left(L h_{\bar{z}}-M h_{z}\right)\left\langle\partial_{z}, \partial_{\bar{z}}\right\rangle}{D} \\
& =\frac{\left(\bar{L} h_{z}-M h_{\bar{z}}\right) L+\left(L h_{\bar{z}}-M h_{z}\right) M}{D} \\
& =\frac{\bar{L} L h_{z}-M L h_{\bar{z}}+L M h_{\bar{z}}-M^{2} h_{z}}{D} \\
& =\frac{|L|^{2}-M^{2}}{D} h_{z} \\
& =h_{z} .
\end{aligned}
$$

De forma análoga, verificamos que $\left\langle\partial_{\bar{z}}, T\right\rangle=h_{\bar{z}}$. Segue de (2.23) que

$$
\nabla_{\partial_{\bar{z}}} S \partial_{z}-\nabla_{\partial_{z}} S \partial_{\bar{z}}=\varepsilon \nu\left(h_{z} \partial_{\bar{z}}-h_{\bar{z}} \partial_{z}\right) .
$$

Fazendo o produto interno da equação anterior com $\partial_{\bar{z}}$, obtemos

$$
\left\langle\nabla_{\partial_{\bar{z}}} S \partial_{z}, \partial_{\bar{z}}\right\rangle-\left\langle\nabla_{\partial_{z}} S \partial_{\bar{z}}, \partial_{\bar{z}}\right\rangle=\varepsilon \nu\left(h_{z}\left\langle\partial_{\bar{z}}, \partial_{\bar{z}}\right\rangle-h_{\bar{z}}\left\langle\partial_{z}, \partial_{\bar{z}}\right\rangle\right)=\varepsilon \nu\left(\bar{L} h_{z}-M h_{\bar{z}}\right),
$$

$\log 0$

$$
\left\langle\nabla_{\partial_{\bar{z}}} S \partial_{z}, \partial_{\bar{z}}\right\rangle-\left\langle\nabla_{\partial_{z}} S \partial_{\bar{z}}, \partial_{\bar{z}}\right\rangle=\varepsilon \nu \alpha
$$

Podemos reescrever a equação acima na forma seguinte

$$
\partial_{\bar{z}}\left\langle S \partial_{z}, \partial_{\bar{z}}\right\rangle-\left\langle S \partial_{z}, \nabla_{\partial_{\bar{z}}} \partial_{\bar{z}}\right\rangle-\partial_{z}\left\langle S \partial_{\bar{z}}, \partial_{\bar{z}}\right\rangle+\left\langle S \partial_{\bar{z}}, \nabla_{\partial_{z}} \partial_{\bar{z}}\right\rangle=\varepsilon \nu \alpha .
$$

Note que

$$
\left\langle S \partial_{z}, \partial_{z}\right\rangle=\left\langle S \partial_{\bar{z}}, \partial_{\bar{z}}\right\rangle=0
$$

e

$$
\left\langle S \partial_{z}, \partial_{\bar{z}}\right\rangle=\left\langle S \partial_{\bar{z}}, \partial_{z}\right\rangle=\rho
$$

Segue de (2.24) que

$$
\rho_{\bar{z}}-\left\langle S \partial_{z}, \nabla_{\partial_{\bar{z}}} \partial_{\bar{z}}\right\rangle+\left\langle S \partial_{\bar{z}}, \nabla_{\partial_{z}} \partial_{\bar{z}}\right\rangle=\varepsilon \nu \alpha
$$

Como

$$
\begin{aligned}
\nabla_{\partial_{z}} \partial_{\bar{z}} & =\Gamma_{12}^{1} \partial_{z}+\Gamma_{12}^{2} \partial_{\bar{z}} \\
\nabla_{\partial_{\bar{z}}} \partial_{\bar{z}} & =\Gamma_{22}^{1} \partial_{z}+\Gamma_{22}^{2} \partial_{\bar{z}}
\end{aligned}
$$


Resulta que

$$
\begin{aligned}
\left\langle S \partial_{z}, \nabla_{\partial_{\bar{z}}} \partial_{\bar{z}}\right\rangle & =\Gamma_{22}^{1}\left\langle S \partial_{z}, \partial_{z}\right\rangle+\Gamma_{22}^{2}\left\langle S \partial_{z}, \partial_{\bar{z}}\right\rangle=\Gamma_{22}^{2} \rho, \\
\left\langle S \partial_{\bar{z}}, \nabla_{\partial_{z}} \partial_{\bar{z}}\right\rangle & =\Gamma_{12}^{1}\left\langle S \partial_{\bar{z}}, \partial_{z}\right\rangle+\Gamma_{12}^{2}\left\langle S \partial_{\bar{z}}, \partial_{\bar{z}}\right\rangle=\Gamma_{12}^{1} \rho .
\end{aligned}
$$

De $(2.25)$

$$
\rho_{\bar{z}}-\Gamma_{22}^{2} \rho+\Gamma_{12}^{1} \rho=\varepsilon \nu \alpha .
$$

Portanto, (2.3) é equivalente a

$$
\frac{\rho_{\bar{z}}}{\rho}+\left(\Gamma_{12}^{1}-\Gamma_{22}^{2}\right)=\varepsilon \alpha \frac{\nu}{\rho} .
$$

Por outro lado, tomando $X=\partial_{z}$, da equação (2.4) segue

$$
\nabla_{\partial_{z}} T=\nu S \partial_{z}
$$

Considerando o produto com $\partial_{\bar{z}}$

$$
\left\langle\nabla_{\partial_{z}} T, \partial_{\bar{z}}\right\rangle=\nu\left\langle S \partial_{z}, \partial_{\bar{z}}\right\rangle .
$$

Por sua vez

$$
\begin{aligned}
\left\langle\nabla_{\partial_{z}} T, \partial_{\bar{z}}\right\rangle & =\partial_{z}\left\langle T, \partial_{\bar{z}}\right\rangle-\left\langle T, \nabla_{\partial_{z}} \partial_{\bar{z}}\right\rangle \\
& =\partial_{z} h_{\bar{z}}-\Gamma_{12}^{1}\left\langle T, \partial_{z}\right\rangle-\Gamma_{12}^{2}\left\langle T, \partial_{\bar{z}}\right\rangle \\
& =h_{z \bar{z}}-\Gamma_{12}^{1} h_{z}-\Gamma_{12}^{2} h_{\bar{z}}
\end{aligned}
$$

e

$$
\nu\left\langle S \partial_{\bar{z}}, \partial_{z}\right\rangle=\nu \rho,
$$

assim

$$
h_{z \bar{z}}=\Gamma_{12}^{1} h_{z}+\Gamma_{12}^{2} h_{\bar{z}}+\nu \rho .
$$

Fazendo o produto de (2.26) com $\partial_{z}$, obtemos

$$
\left\langle\nabla_{\partial_{z}} T, \partial_{z}\right\rangle=\nu\left\langle S \partial_{z}, \partial_{z}\right\rangle .
$$

Procedendo de forma análoga

$$
\begin{aligned}
\left\langle\nabla_{\partial_{z}} T, \partial_{z}\right\rangle & =\partial_{z}\left\langle T, \partial_{z}\right\rangle-\left\langle T, \nabla_{\partial_{z}} \partial_{z}\right\rangle \\
& =\partial_{z} h_{z}-\left\langle T, \Gamma_{11}^{1} \partial_{z}+\Gamma_{11}^{2} \partial_{\bar{z}}\right\rangle \\
& =h_{z z}-\Gamma_{11}^{1} h_{z}-\Gamma_{11}^{2} h_{\bar{z}}
\end{aligned}
$$


Como

$$
\left\langle S \partial_{z}, \partial_{z}\right\rangle=0
$$

obtemos

$$
h_{z z}=\Gamma_{11}^{1} h_{z}+\Gamma_{11}^{2} h_{\bar{z}}
$$

As equações (2.16) e (2.17) anteriores são equivalentes a (2.4).

Agora, escrevendo (2.5) para $X=\partial_{\bar{z}}$ e usando (2.20) e (2.21), temos

$$
\begin{aligned}
\nu_{\bar{z}} & =-\left\langle S \partial_{\bar{z}}, T\right\rangle \\
& =-\left\langle S \partial_{\bar{z}}, \frac{1}{D}\left(\alpha \partial_{z}+\bar{\alpha} \partial_{\bar{z}}\right)\right\rangle \\
& =-\frac{\alpha}{D}\left\langle S \partial_{\bar{z}}, \partial_{z}\right\rangle \\
& =-\frac{\alpha}{D} \rho \\
& =\frac{\alpha K}{\rho} .
\end{aligned}
$$

Essa é expressão equivalente a (2.5).

Finalmente, de (2.21) e (2.22) temos

$$
\begin{aligned}
\langle T, T\rangle & =\left\langle T, \frac{1}{D}\left(\alpha \partial_{z}+\bar{\alpha} \partial_{\bar{z}}\right)\right\rangle \\
& =\frac{1}{D}\left[\alpha\left\langle T, \partial_{z}\right\rangle+\bar{\alpha}\left\langle T, \partial_{\bar{z}}\right\rangle\right] \\
& =\frac{1}{D}\left(\alpha h_{z}+\bar{\alpha} h_{\bar{z}}\right)
\end{aligned}
$$

De $(2.6)$

$$
\frac{1}{D}\left(\alpha h_{z}+\bar{\alpha} h_{\bar{z}}\right)+\nu^{2}=1
$$

O foco deste trabalho será em superfícies com curvatura Gaussiana constante. Assim, de agora em diante suporemos que $\psi$ tem curvatura Gaussiana constante $K(I)$. A fim de obter algumas estimativas para essas superfícies, calculamos o Laplaciano de $h$ e $\nu$ com respeito a segunda forma fundamental.

\section{Proposição 2.3.}

Seja $\psi: \Sigma \rightarrow \mathbb{M}^{2}(\varepsilon) \times \mathbb{R}$ uma imersão com curvatura Gaussiana constante $K(I)$ e curvatura 
extrínseca positiva. Vamos considerar um parâmetro conforme z para sua segunda forma fundamental tal que

$$
\begin{gathered}
I=\langle d \psi, d \psi\rangle=L d z^{2}+2 M|d z|^{2}+\bar{L} d \bar{z}^{2} \\
I I=\langle d \psi,-d N\rangle=2 \rho|d z|^{2}, \quad \rho>0 .
\end{gathered}
$$

Então temos

$$
\begin{aligned}
& h_{z \bar{z}}=(K(I)-\varepsilon) \frac{\nu \rho}{K}, \\
& \nu_{z \bar{z}}=\varepsilon \nu \frac{|\alpha|^{2}}{D}-\nu M K .
\end{aligned}
$$

Demonstração. Como

$$
D_{\bar{z}}=L_{\bar{z}} \bar{L}+L \bar{L}_{\bar{z}}-2 M M_{\bar{z}}
$$

então (1.63) e (2.15) permite-nos obter

$$
\frac{D_{\bar{z}}}{2 D}-\frac{\rho_{\bar{z}}}{\rho}=2 \Gamma_{12}^{1}-\varepsilon \alpha \frac{\nu}{\rho} .
$$

Agora, diferenciando (2.20) com respeito a $\bar{z}$, resulta

$$
\frac{D_{\bar{z}}}{2 D}-\frac{\rho_{\bar{z}}}{\rho}=-\frac{K_{\bar{z}}}{2 K}
$$

e assim obtemos de (2.29) e (2.30)

$$
\Gamma_{12}^{1}=-\frac{K_{\bar{z}}}{4 K}+\varepsilon \alpha \frac{\nu}{2 \rho} .
$$

Por outro lado, já que a curvatura Gaussiana de $\psi$ é constante, diferenciando a equação de Gauss (2.14), obtemos

$$
K_{\bar{z}}=-2 \varepsilon \nu \nu_{\bar{z}}
$$

e usando (2.18)

$$
K_{\bar{z}}=-2 \varepsilon \nu \frac{\alpha K}{\rho} .
$$

Portanto de (2.31),

$$
\Gamma_{12}^{1}=\varepsilon \frac{\nu \alpha}{\rho} .
$$


De (2.17), tendo em mente que $\overline{\Gamma_{12}^{1}}=\Gamma_{12}^{2}$, temos

$$
\begin{aligned}
h_{z \bar{z}} & =\Gamma_{12}^{1} h_{z}+\Gamma_{12}^{2} h_{\bar{z}}+\nu \rho \\
& =\varepsilon \frac{\nu \alpha}{\rho} h_{z}+\varepsilon \frac{\nu \bar{\alpha}}{\rho} h_{\bar{z}}+\nu \rho \\
& =\nu \rho\left(1+\varepsilon \frac{\alpha h_{z}+\bar{\alpha} h_{\bar{z}}}{\rho^{2}}\right)
\end{aligned}
$$

e usando (2.20) e (2.19)

$$
h_{z \bar{z}}=\nu \rho\left(1+\varepsilon \frac{D\left(1-\nu^{2}\right)}{\rho^{2}}\right)=\frac{\nu \rho}{K}\left(K-\varepsilon\left(1-\nu^{2}\right)\right) .
$$

Agora, se diferenciarmos (2.18) e usarmos (2.20), (2.30) e (2.32) então temos

$$
\begin{aligned}
\nu_{z \bar{z}} & =\frac{K \alpha_{z}}{\rho}+\frac{K_{z} \alpha}{\rho}-\frac{K \alpha \rho_{z}}{\rho^{2}} \\
& =\frac{K \alpha_{z}}{\rho}+\frac{K_{z} \alpha}{\rho}-\frac{K \alpha}{\rho}\left(\frac{D_{z}}{2 D}+\frac{K_{z}}{2 K}\right) \\
& =\frac{K \alpha_{z}}{\rho}+\frac{K_{z} \alpha}{\rho}-\frac{K \alpha D_{z}}{2 \rho D}-\frac{K_{z} \alpha}{2 \rho} \\
& =\frac{K \alpha_{z}}{\rho}+\frac{K_{z} \alpha}{2 \rho}-\frac{\alpha K}{\rho} \frac{D_{z}}{2 D} \\
& =\frac{K \alpha_{z}}{\rho}+\frac{\alpha}{2 \rho}\left(-2 \varepsilon \nu \frac{\bar{\alpha} K}{\rho}\right)-\frac{\alpha K}{\rho} \frac{D_{z}}{2 D} \\
& =\frac{K \alpha_{z}}{\rho}-\varepsilon \nu \frac{|\alpha|^{2} K}{\rho^{2}}-\frac{\alpha K}{\rho} \frac{D_{z}}{2 D} \\
& =\frac{K}{\rho} \alpha_{z}+\varepsilon \nu \frac{|\alpha|^{2}}{D}-\frac{\alpha K}{\rho} \frac{D_{z}}{2 D},
\end{aligned}
$$

isto é,

$$
\nu_{z \bar{z}}=\frac{K}{\rho} \alpha_{z}+\varepsilon \nu \frac{|\alpha|^{2}}{D}-\frac{\alpha K}{\rho} \frac{D_{z}}{2 D} .
$$

Derivando

$$
\alpha=\bar{L} h_{z}-M h_{\bar{z}}=\left\langle\psi_{\bar{z}}, \psi_{\bar{z}}\right\rangle h_{z}-\left\langle\psi_{z}, \psi_{\bar{z}}\right\rangle h_{\bar{z}}
$$

em relação a $\bar{z}$, obtemos

$$
\alpha_{z}=2\left\langle\psi_{z \bar{z}}, \psi_{\bar{z}}\right\rangle h_{z}+\bar{L} h_{z z}-\left\langle\psi_{z z}, \psi_{\bar{z}}\right\rangle h_{\bar{z}}-\left\langle\psi_{z}, \psi_{z \bar{z}}\right\rangle h_{\bar{z}}-M h_{z \bar{z}} .
$$

Usando (2.16) e (2.17), obtemos

$$
\begin{aligned}
\alpha_{z}= & 2\left(\Gamma_{12}^{1} M+\Gamma_{12}^{2} \bar{L}\right) h_{z}+\bar{L}\left(\Gamma_{11}^{1} h_{z}+\Gamma_{11}^{2} h_{\bar{z}}\right)-\left(\Gamma_{11}^{1} M+\Gamma_{11}^{2} \bar{L}\right) h_{\bar{z}} \\
& -\left(\Gamma_{12}^{1} L+\Gamma_{12}^{2} M\right) h_{\bar{z}}-L\left(\Gamma_{12}^{1} h_{z}+\Gamma_{12}^{2} h_{\bar{z}}+\rho \nu\right) \\
= & \alpha\left(\Gamma_{12}^{2}+\Gamma_{11}^{1}\right)+\alpha \Gamma_{12}^{2}-\bar{\alpha} \Gamma_{12}^{1}-\nu \rho M .
\end{aligned}
$$


Observamos que de (2.33), podemos concluir que $\alpha \Gamma_{12}^{2}-\bar{\alpha} \Gamma_{12}^{1}=0$, e conjugando (1.63), obtemos $\Gamma_{12}^{2}+\Gamma_{11}^{1}=\frac{D_{z}}{2 D}$. Assim,

$$
\alpha_{z}=\alpha \frac{D_{z}}{2 D}-\nu \rho M
$$

Então resulta de (2.34)

$$
\nu_{z \bar{z}}=\frac{K}{\rho}\left(\alpha \frac{D_{z}}{2 D}-\nu \rho M\right)+\varepsilon \nu \frac{|\alpha|^{2}}{D}-\frac{\alpha K}{\rho} \frac{D_{z}}{2 D}=\varepsilon \nu \frac{|\alpha|^{2}}{D}-\nu M K .
$$




\section{Estimativas de altura}

Neste capítulo, obteremos estimativas de altura ótimas para gráficos em $\mathbb{M}^{2} \times \mathbb{R}$. A altura do gráfico é medida em relação ao slice $\mathbb{M}^{2}(\varepsilon) \times\{0\}$. Veremos também que o limite máximo destas estimativas são atingidos se, e somente se, a imersão for um hemisfério de uma superfície completa de rotação.

\subsection{Estimativas de altura para superfícies de curvatura Gaussiana constante}

Vamos considerar a forma quadrática

$$
Q d z^{2}=\left((K(I)-\varepsilon)\left\langle\psi_{z}, \psi_{z}\right\rangle+\varepsilon h_{z}^{2}\right) d z^{2}
$$

que está bem definida para superfícies de curvatura Gaussiana constante e curvatura extrínseca positiva (ver [2]).

\section{Proposição 3.1.}

Seja $\Sigma$ uma superfície de curvatura Gaussiana constante em $\mathbb{M}^{2} \times \mathbb{R}$, então a forma quadrática

$$
Q d z^{2}=\left((K(I)-\varepsilon)\left\langle\psi_{z}, \psi_{z}\right\rangle+\varepsilon h_{z}^{2}\right) d z^{2}
$$

é uma forma quadrática holomorfa. 
Demonstração. De (2.20), (2.22), (2.27) e (2.31) obtemos

$$
\begin{aligned}
Q_{\bar{z}} & =2(K(I)-\varepsilon)\left\langle\psi_{z \bar{z}}, \psi_{z}\right\rangle+2 \varepsilon h_{z} h_{z \bar{z}} \\
& =2(K(I)-\varepsilon)\left(\Gamma_{12}^{1} L+\Gamma_{12}^{2} M\right)+2 \varepsilon h_{z}(K(I)-\varepsilon) \frac{\nu \rho}{K} \\
& =2 \varepsilon(K(I)-\varepsilon)\left(\frac{\nu \alpha}{\rho} L+\frac{\nu \bar{\alpha}}{\rho} M-\frac{\nu D}{\rho} h_{z}\right) \\
& =2 \varepsilon(K(I)-\varepsilon) \frac{\nu}{\rho}\left(\alpha L+\bar{\alpha} M-D h_{z}\right) \\
& =2 \varepsilon(K(I)-\varepsilon) \frac{\nu}{\rho}\left(\left(\bar{L} h_{z}-M h_{\bar{z}}\right) L+\left(L h_{\bar{z}}-M h_{z}\right) M-\left(|L|^{2}-M^{2}\right) h_{z}\right) \\
& =2 \varepsilon(K(I)-\varepsilon) \frac{\nu}{\rho}\left(|L|^{2} h_{z}-L M_{\bar{z}}+L M h_{\bar{z}}-M^{2} h_{z}-\left(|L|^{2}-M^{2}\right) h_{z}\right) \\
& =2 \varepsilon(K(I)-\varepsilon) \frac{\nu}{\rho}\left(\left(|L|^{2}-M^{2}\right) h_{z}-\left(|L|^{2}-M^{2}\right) h_{z}\right) \\
& =0 .
\end{aligned}
$$

Isto é, $Q d z^{2}$ é uma forma quadrática holomorfa.

Também temos como uma consequência o seguinte corolário:

\section{Corolário 3.1.}

Seja $\Sigma$ uma superfície satisfazendo

1. $\Sigma$ é uma superfície plana em $\mathbb{H}^{2} \times \mathbb{R}$, que é localmente um gráfico sobre $\mathbb{H}^{2}$. Então a projeção $\eta: \mathbb{H}^{2} \times \mathbb{R} \rightarrow \mathbb{H}^{2} \times\{0\} \equiv \mathbb{H}^{2}$ é uma aplicação harmônica para a segunda forma fundamental.

2. $\Sigma$ é uma superfície de curvatura Gaussiana constante igual a $1 \mathrm{em} \mathbb{S}^{2} \times \mathbb{R}$ com $\|T\| \neq 0$ em toda a superfície. Então a função altura é harmônica para a segunda forma fundamental.

Demonstração. Observamos que, de (2.2) e (2.6), uma superfície plana em $\mathbb{H}^{2} \times \mathbb{R}$ tem curvatura extrínseca positiva se, e somente se, $\|T\| \neq 1$ em toda a superfície. Ou equivalentemente, a superfície é localmente um gráfico sobre $\mathbb{H}^{2}$.

Adicionalmente, para a superfície plana $\Sigma$

$$
Q d z^{2}=\left(\left\langle\psi_{z}, \psi_{z}\right\rangle-h_{z}^{2}\right) d z^{2}=\left\langle\eta_{z}, \eta_{z}\right\rangle d z^{2}
$$

De onde

$$
Q_{\bar{z}}=2\left\langle\eta_{z \bar{z}}, \eta_{z}\right\rangle
$$


é holomorfa. Isto é, $\eta$ é uma aplicação harmônica. De fato, escrevendo $\eta_{z}=\eta_{u}-i \eta_{v}$, segue de $(3.2)$

$$
\left\langle\eta_{z \bar{z}}, \eta_{u}\right\rangle-i\left\langle\eta_{z \bar{z}}, \eta_{v}\right\rangle=0
$$

de onde $\left\langle\eta_{z \bar{z}}, \eta_{u}\right\rangle=\left\langle\eta_{z \bar{z}}, \eta_{v}\right\rangle=0$, isto é, $\eta_{z \bar{z}}$ é normal a $\mathbb{H}^{2}$, e portanto harmônica (veja [10], exemplo 9).

A prova é análoga para o segundo caso.

Esses resultados devem ser entendidos como um análogo para o fato que $\eta$ e $h$ são aplicações harmônicas para a métrica induzida de uma superfície mínima em $\mathbb{H}^{2} \times \mathbb{R}$. Mais geralmente, a função altura é uma aplicação harmônica para a métrica induzida de uma superfície mínima no espaço produto $\mathbb{M}^{2} \times \mathbb{R}$.

Nas nossas estimativas de altura veremos que os casos extremos são superfícies completas de curvatura Gaussiana constante em $\mathbb{M}^{2}(\varepsilon) \times \mathbb{R}$ (veja [2]). Assim, vamos descrevê-los brevemente.

Exemplo 3.1 $\left(K(I)\right.$ - superfícies em $\mathbb{H}^{2} \times \mathbb{R}$.). Seja $K(I)$ uma constante positiva e

$$
\psi(u, v)=(\cosh k(v), \operatorname{senh} k(v) \cos u, \operatorname{senh} k(v) \operatorname{sen} u, h(v))
$$

a superfície de revolução em $\mathbb{H}^{2} \times \mathbb{R}$ dada por

$$
\begin{gathered}
k(v)=\operatorname{arcsenh}\left(\frac{1}{\sqrt{K(I)}} \operatorname{sen}(\sqrt{K(I)} v)\right) \text { e } \\
h(v)=-\sqrt{\frac{1+K(I)}{K(I)}} \arctan \left(\frac{\cos (\sqrt{K(I)} v)}{\sqrt{K(I)+\operatorname{sen}^{2}(\sqrt{K(I)} v)}}\right)
\end{gathered}
$$

$\operatorname{com} v \in\left[0, \frac{\pi}{\sqrt{K(I)}}\right]$.

Então, $\psi$ é, a menos de isometrias, a parametrização da única superfície completa com curvatura Gaussiana constante $K(I)$ em $\mathbb{H}^{2} \times \mathbb{R}$.

Exemplo 3.2 $\left(K(I)\right.$-superfícies completas em $\mathbb{S}^{2} \times \mathbb{R}$.). Vamos considerar uma constante $K(I)>1 \mathrm{e}$

$$
\psi(u, v)=(\operatorname{sen} k(v), \cos k(v) \cos u, \cos k(v) \operatorname{sen} u, h(v))
$$


a superfície de revolução em $\mathbb{S}^{2} \times \mathbb{R}$ dada por

$$
\begin{gathered}
k(v)=\arccos \left(\frac{1}{\sqrt{K(I)}} \operatorname{sen}(\sqrt{K(I)} v)\right) \text { e } \\
h(v)=-\sqrt{\frac{K(I)-1}{K(I)}} \log \left(\frac{\cos (\sqrt{K(I)} v)+\sqrt{K(I)-\operatorname{sen}^{2}(\sqrt{K(I)} v)}}{1+\sqrt{K(I)}}\right)
\end{gathered}
$$

$\operatorname{com} v \in\left[0, \frac{\pi}{\sqrt{K(I)}}\right]$

Então, $\psi$ é, a menos de isometrias, a parametrização da única superfície completa com curvatura Gaussiana constante $K(I)$ em $\mathbb{S}^{2} \times \mathbb{R}$.

Provaremos que nossas estimativas alturas são alcançadas quando a forma quadrática $Q d z^{2}$ é identicamente nula. Assim, a fim de caracterizar esses exemplos temos o seguinte teorema. Observamos que esse resultado é local e uma versão global é dada em $[2]$.

\section{Teorema 3.1.}

Seja $\psi: \Sigma \rightarrow \mathbb{M}^{2}(\varepsilon) \times \mathbb{R}$ uma imersão de curvatura Gaussiana constante $K(I)>0$ se $\varepsilon=-1$ (respec. $K(I)>1$ se $\varepsilon=1$ ). Vamos assumir que $Q d z^{2}$ é identicamente nula sobre $\Sigma$. Então $\psi$ é uma parte de uma $K(I)$-superfície completa.

Demonstração. Para uma exposição clara vamos dividir essa demonstração em duas partes. Primeiramente, mostraremos que a imersão deve ser helicoidal, isto é, $\psi$ é invariante sob um grupo contínuo de isometrias do espaço ambiente. Então, na segunda parte, demonstraremos que as órbitas desse movimento helicoidal são círculos sobre os slices $\mathbb{M}^{2}(\varepsilon) \times\left\{h_{0}\right\}$. Assim, concluiremos que $\psi$ é uma superfície de revolução.

Seguindo [2], definimos a métrica

$$
A=I+\frac{\varepsilon}{K(I)-\varepsilon} d h^{2}
$$

Note que

$$
A=\left(L+\frac{\varepsilon}{K(I)-\varepsilon} h_{z}^{2}\right) d z^{2}+2\left(M+\frac{\varepsilon}{K(I)-\varepsilon} h_{z} h_{\bar{z}}\right)|d z|^{2}+\left(\bar{L}+\frac{\varepsilon}{K(I)-\varepsilon} h_{\bar{z}}^{2}\right) d \bar{z}^{2}
$$

Como $Q d z^{2} \equiv 0$, resulta que a $(2,0)$ - parte de $A$ com respeito a $I I$ se anula. De (2.2) e (2.6), $K$ é positiva e então $I I$ é definida. Portanto, $A$ e $I I$ são conformes. Em particular, existe uma função $\lambda$ sobre $S$ tal que $I I=\lambda A$. E, de ([2], Lema 1$), \lambda^{2}=K(I)-\varepsilon$. 
Seja $(u, v)$ coordenadas duplamente ortogonais para a primeira e segunda formas fundamentais, isto é,

$$
\begin{aligned}
& I=E d u^{2}+G d v^{2}, \\
& I I=k_{1} E d u^{2}+k_{2} G d v^{2},
\end{aligned}
$$

onde $k_{1}$ e $k_{2}$ são as curvaturas principais da imersão $\psi$. Lembre que essas coordenadas estão disponíveis na vizinhança de todo ponto não umbílico bem como sobre o interior do conjunto dos pontos umbílicos. Segue que, consideraremos esses pontos e, usando que esse conjunto é denso em $\Sigma$, deduziremos que as propriedades obtidas podem ser estendidas para toda superfície por continuidade.

Já que

$$
\begin{aligned}
A= & \left(E+\frac{\varepsilon}{K(I)-\varepsilon} h_{u}^{2}\right) d u^{2} \\
& +2 \frac{\varepsilon}{K(I)-\varepsilon} h_{u} h_{v} d u d v+\left(G+\frac{\varepsilon}{K(I)-\varepsilon} h_{v}^{2}\right) d v^{2}
\end{aligned}
$$

$\mathrm{e} I I=\sqrt{K(I)-\varepsilon} A$ temos

$$
\begin{aligned}
k_{1} E & =\sqrt{K(I)-\varepsilon}\left(E+\frac{\varepsilon}{K(I)-\varepsilon} h_{u}^{2}\right), \\
0 & =h_{u} h_{v}, \\
k_{2} G & =\sqrt{K(I)-\varepsilon}\left(G+\frac{\varepsilon}{K(I)-\varepsilon} h_{v}^{2}\right) .
\end{aligned}
$$

De (3.4) vemos facilmente que o conjunto dado pela união do interior do conjunto onde $h_{u}=0$ e a do interior do conjunto onde $h_{v}=0$ é denso. De fato, se $h_{v}(p) \neq 0$ para algum ponto $p \in \Sigma$, existe uma vizinhança $V$ de $p$ onde $h_{v} \neq 0$. Mas como $h_{v} h_{v}=0$, segue que $h_{u}=0 \mathrm{em} V$. Consequentemente, podemos assumir que $h_{u} \equiv 0$ na vizinhança onde $(u, v)$ são tomados.

Então, de (3.3), a curvatura principal $k_{1}=\sqrt{K(I)-\varepsilon}$ é uma constante positiva. Além disso, de (2.5)

$$
\nu_{u}=d \nu\left(\partial_{u}\right)=-\left\langle S \partial_{u}, T\right\rangle=-\left\langle k_{1} \partial_{u}, T\right\rangle=-k_{1} h_{u}=0
$$

Portanto, da equação de Gauss (3.1),

$$
K(I)=k_{1} k_{2}+\varepsilon \nu^{2} .
$$


Derivando a equação acima em relação a $u$, obtemos $0=k_{1}\left(k_{2}\right)_{u}$, de onde $\left(k_{2}\right)_{u}=0$.

Agora, (3.5) pode ser reescrita como

$$
k_{2} G=k_{1}\left(G+\frac{\varepsilon}{k_{1}^{2}} h_{v}^{2}\right)
$$

de onde

$$
k_{1}\left(k_{2}-k_{1}\right) G=\varepsilon h_{v}^{2}
$$

Uma vez que estamos considerando uma vizinhança sem pontos umbílicos ou vizinhança totalmente umbílica, observamos que se $k_{1} \equiv k_{2}$ então, de (3.6), $h_{v}$ também é identicamente nula. Portanto, $h$ deve ser constante, isto é, a superfície estaria sobre um slice e então $K(I)=\varepsilon$, o que é uma contradição.

Consequentemente, $k_{1} \neq k_{2}$ em nossa vizinhança e, de (3.6), $G_{u} \equiv 0$.

Se considerarmos a equação de Codazzi (2.3) para $X=\partial_{u}$ e $Y=\partial_{v}$, obtemos

$$
\begin{aligned}
\left(k_{2}-k_{1}\right) \nabla_{\partial_{u}} \partial_{v} & =k_{2} \nabla_{\partial_{u}} \partial_{v}-k_{1} \nabla_{\partial_{u}} \partial_{v} \\
& =k_{2} \nabla_{\partial_{u}} \partial_{v}-k_{1} \nabla_{\partial_{v}} \partial_{u} \\
& =\nabla_{\partial_{u}} k_{2} \partial_{v}-\nabla_{\partial_{v}} k_{1} \partial_{u} \\
& =\nabla_{\partial_{u}} S \partial_{v}-\nabla_{\partial_{v}} S \partial_{u} \\
& =\varepsilon \nu\left(\left\langle\partial_{v}, T\right\rangle \partial_{u}-\left\langle\partial_{u}, T\right\rangle \partial_{v}\right) \\
& =\varepsilon \nu\left(h_{v} \partial_{u}-h_{u} \partial_{v}\right) \\
& =\varepsilon \nu h_{v} \partial_{u} .
\end{aligned}
$$

Além disso, de (1.72) e (1.73)

$$
\begin{aligned}
\nabla_{\partial_{u}} \partial_{v} & =\Gamma_{12}^{1} \partial_{u}+\Gamma_{12}^{2} \partial_{v} \\
& =\frac{E_{v}}{2 E} \partial_{u}+\frac{G_{u}}{2 G} \partial_{v} \\
& =\frac{E_{v}}{2 E} \partial_{u}
\end{aligned}
$$

e, como $\nabla_{\partial_{u}} \partial_{v}=\frac{\varepsilon \nu h_{v}}{k_{2}-k_{1}} \partial_{u}$, então

$$
(\log E)_{u v}=\left(\frac{E_{v}}{E}\right)_{u}=\frac{\left(\varepsilon \nu_{u} h_{v}+\varepsilon \nu h_{v u}\right)\left(k_{2}-k_{1}\right)-\varepsilon \nu h_{v}\left(k_{2}\right)_{u}}{\left(k_{2}-k_{1}\right)^{2}}=0,
$$

isto é, a função $E(u, v)$ pode ser escrita como $E(u, v)=E_{1}(u) E_{2}(v)$ para funções positivas $E_{1}$ e $E_{2}$. 
Finalmente, tomamos novos parâmetros $(x, y)$ tais que

$$
d x=\sqrt{E_{1}(u)} d u, \quad y=v .
$$

Então, a primeira forma fundamental, a segunda forma fundamental, $h$ e $\nu$ somente dependem de $y$, isto é, as funções $E, G, k_{1}, k_{2}$ e $\nu$ não dependem de $(x, y)$, mas somente de $y$.

Consequentemente, a imersão $\psi(x, y)$ e $\varphi(x, y)=\psi\left(x+x_{0}, y\right)$, para um adequado $x_{0}$, tem as mesmas funções $E, G, k_{1}, k_{2}, h$ e $\nu$. Então, $\psi(x, y)$ e $\psi\left(x+x_{0}, y\right)$ somente diferem por uma isometria do espaço ambiente para cada $x_{0}$ (veja [4]), isto é, $\psi$ é helicoidal e as órbitas são dadas por $\beta(t)=\psi(x+t, y)$.

Na segunda parte da prova, mostraremos que $\psi$ é uma superfície de rotação. Primeiramente, observamos que $\beta(t)$ está contida em um slice porque a função altura somente depende de $y$.

Em particular,

$$
\beta(t) \subset \mathbb{M}^{2}(\varepsilon) \times\{y\} \equiv \mathbb{M}^{2}(\varepsilon)
$$

é invariante sob um grupo de isometrias de $\mathbb{M}^{2}(\varepsilon)$. Então, a curvatura de $\beta$ em $\mathbb{M}^{2}(\varepsilon)$ é constante.

Portanto, se $\varepsilon=1$ então $\beta$ está contida sobre um círculo de $\mathbb{S}^{2}$. De fato, seja $\alpha: I \rightarrow \mathbb{S}^{2} \subset \mathbb{R}^{3}$. Note que

$$
\nabla_{\alpha^{\prime}} \alpha^{\prime}=\alpha^{\prime \prime}-\left\langle\alpha^{\prime \prime}, \alpha\right\rangle \alpha
$$

Como $\left\langle\alpha^{\prime}, \alpha\right\rangle=0$, temos $\left\langle\alpha^{\prime \prime}, \alpha\right\rangle+\left\langle\alpha^{\prime}, \alpha^{\prime}\right\rangle=0$, de onde $\left\langle\alpha^{\prime \prime}, \alpha\right\rangle=-1$. De (3.7)

$$
\nabla_{\alpha^{\prime}} \alpha^{\prime}=\alpha^{\prime \prime}+\alpha
$$

Consequentemente

$$
\begin{aligned}
k^{2} & =\left|\nabla_{\alpha^{\prime}} \alpha^{\prime}\right|^{2} \\
& =\left\langle\alpha^{\prime \prime}, \alpha^{\prime \prime}\right\rangle+2\left\langle\alpha^{\prime \prime}, \alpha\right\rangle+\langle\alpha, \alpha\rangle \\
& =\left\langle\alpha^{\prime \prime}, \alpha^{\prime \prime}\right\rangle-1,
\end{aligned}
$$

$\operatorname{logo}\left\langle\alpha^{\prime \prime}, \alpha^{\prime \prime}\right\rangle=k^{2}+1, k$ constante. 
Vendo $\alpha$ como curva em $\mathbb{R}^{3}, \alpha^{\prime \prime}=k_{E} n$, de onde $k_{E}^{2}=k^{2}+1 \neq 0$ constante.

Decorre de $\left\langle\alpha^{\prime}, \alpha\right\rangle=0$, que $\left\langle\alpha^{\prime \prime}, \alpha\right\rangle+\left\langle\alpha^{\prime}, \alpha^{\prime}\right\rangle=0$. Assim

$$
k_{E}\langle n, \alpha\rangle=-1
$$

portanto

$$
\langle n, \alpha\rangle=-\frac{1}{k_{E}}
$$

Derivando esta última equação e usando as fórmulas de Frenet

$$
\left\langle-k_{E} t-\tau_{E} b, \alpha\right\rangle+\langle n, t\rangle=0
$$

Segue que $\tau_{E}\langle b, \alpha\rangle=0$. Suponha $\tau_{E} \neq 0$, então $\langle b, \alpha\rangle=0$, de onde

$$
\left\langle b^{\prime}, \alpha\right\rangle+\langle b, t\rangle=0
$$

$\operatorname{logo}-\tau_{E}\langle n, \alpha\rangle=0$. Mas $\langle n, \alpha\rangle=-\frac{1}{k_{E}}$, o que nos dá uma contradição. Logo $\tau_{E}=0$. Assim $\tau_{E}=0$ e $k_{E}$ constante implicam que $\alpha$ é um círculo.

Por outro lado, se $\varepsilon=-1, \beta$ está contida em um círculo de $\mathbb{H}^{2}$ se, e somente se, sua curvatura é maior que 1 . De fato, seja $\alpha: I \rightarrow \mathbb{H}^{2} \subset \mathbb{R}_{1}^{3}$, tal que $\langle\alpha, \alpha\rangle=-1$.

Escrevendo

$$
\alpha^{\prime \prime}=\nabla_{\alpha^{\prime}} \alpha^{\prime}+\lambda \alpha
$$

e fazendo o produto com $\alpha$, obtemos $\left\langle\alpha^{\prime \prime}, \alpha\right\rangle=-\lambda$, de onde concluímos que

$$
\nabla_{\alpha^{\prime}} \alpha^{\prime}=\alpha^{\prime \prime}+\left\langle\alpha^{\prime \prime}, \alpha\right\rangle \alpha
$$

Decorre de $\left\langle\alpha^{\prime}, \alpha\right\rangle=0$ que $\left\langle\alpha^{\prime \prime}, \alpha\right\rangle=-\left\langle\alpha^{\prime}, \alpha^{\prime}\right\rangle=-1$, assim

$$
\nabla_{\alpha^{\prime}} \alpha^{\prime}=\alpha^{\prime \prime}-\alpha
$$

e

$$
\begin{aligned}
k^{2} & =\left|\nabla_{\alpha^{\prime}} \alpha^{\prime}\right| \\
& =\left\langle\alpha^{\prime \prime}, \alpha^{\prime \prime}\right\rangle-2\left\langle\alpha^{\prime \prime}, \alpha\right\rangle+\langle\alpha, \alpha\rangle \\
& =\left\langle\alpha^{\prime \prime}, \alpha^{\prime \prime}\right\rangle+2-1 \\
& =\left\langle\alpha^{\prime \prime}, \alpha^{\prime \prime}\right\rangle+1 .
\end{aligned}
$$


Consequentemente

$$
\left\langle\alpha^{\prime \prime}, \alpha^{\prime \prime}\right\rangle=k^{2}-1
$$

Vendo $\alpha$ como uma curva em $\mathbb{L}^{3}$. Se $k^{2}-1>0$, então $\alpha^{\prime \prime}=t^{\prime}$ é tipo espaço e $k_{L}=\left|\alpha^{\prime \prime}\right|=\sqrt{k^{2}-1} \neq 0$. Temos

$$
\begin{aligned}
t^{\prime} & =k_{L} n, \\
n^{\prime} & =-k_{L} t+\tau_{L} b, \\
b^{\prime} & =\tau_{L} n .
\end{aligned}
$$

Novamente, como $\left\langle\alpha^{\prime}, \alpha\right\rangle=0$, concluímos que $\left\langle\alpha^{\prime \prime}, \alpha\right\rangle=-1 \mathrm{e}$

$$
\langle n, \alpha\rangle=-\frac{1}{k_{L}}
$$

Supondo $k_{L}$ constante e derivando (3.8)

$$
\left\langle-k_{L} t+\tau_{L} b, \alpha\right\rangle+\langle n, t\rangle=0
$$

$\operatorname{logo} \tau_{L}\langle b, \alpha\rangle=0$ e, de forma análoga ao caso anterior, concluímos que $\tau_{L}=0$. Assim, se $n$ e $t$ são tipo espaço, então $b$ é tipo tempo constante, então a curva está contida num plano euclidiano, de onde está num círculo.

Para o caso em que a curva está contida em $\mathbb{H}^{2}$, um cálculo fácil nos dá

$$
\nabla_{\partial_{x}} \partial_{x}=-\frac{1}{2} \frac{E_{y}}{G} \partial_{y}+k_{1} E N
$$

onde $\nabla$ denota a conexão de Levi-Civita em $\mathbb{H}^{2} \times \mathbb{R}$. Além disso, $\beta$ pode ser parametrizado pelo comprimento de arco como $\beta(s)=\psi\left(x+\frac{s}{\sqrt{E(y)}}, y\right)$. Então, o quadrado de sua curvatura é dado por

$$
\begin{aligned}
\left\langle\nabla_{\beta^{\prime}(s)} \beta^{\prime}(s), \nabla_{\beta^{\prime}(s)} \beta^{\prime}(s)\right\rangle & =\left\langle-\frac{1}{2} \frac{1}{E} \frac{E_{y}(y)}{G(y)} \psi_{y}+k_{1} E N,-\frac{1}{2} \frac{1}{E} \frac{E_{y}(y)}{G(y)} \psi_{y}+k_{1} E N\right\rangle \\
& =\frac{1}{4} \frac{1}{E(y)^{2}}\left(\frac{E_{y}(y)^{2}}{G(y)}+k_{1}^{2} E(y)^{2}\right) \geq k_{1}^{2} \\
& =K(I)+1>1 .
\end{aligned}
$$

Assim, $\beta$ está contida em um círculo em qualquer caso e $\psi$ deve ser uma superfície de rotação. Finalmente, a prova finaliza como consequência do próxima lema. 


\section{Lema 3.1.}

Seja $\psi: \Sigma \rightarrow \mathbb{M}^{2}(\varepsilon) \times \mathbb{R}$ uma superfície de rotação tal que a curvatura principal $k_{1}$ associada com seus paralelos coincidem. Se $k_{1}>1$ para $\varepsilon=-1$ ou $k_{1}>0$ para $\varepsilon=1$ então, a menos de isometria, $\psi$ é um pedaço da superfície completa de curvatura Gaussiana constante descritas no Exemplo (3.1) ou Exemplo (3.2).

Demonstração. Vamos parametrizar

$$
\begin{array}{ll}
\psi(u, v)=(\cosh k(v), \operatorname{senh} k(v) \cos u, \operatorname{senh} k(v) \operatorname{sen} u, h(v)), & \text { se } \quad \varepsilon=-1, \\
\psi(u, v)=(\operatorname{sen} k(v), \cos k(v) \cos u, \cos k(v) \operatorname{sen} u, h(v)), & \text { se } \quad \varepsilon=1,
\end{array}
$$

$\operatorname{com} k^{\prime}(v)^{2}+h^{\prime}(v)^{2}=1$.

Então, um simples cálculo nos dá que as curvaturas principais associadas com os paralelos são

$$
\begin{array}{ll}
k_{1}=h^{\prime}(v) \operatorname{coth}(k(v)), & \text { se } \quad \varepsilon=-1, \\
k_{1}=h^{\prime}(v) \tan (k(v)), & \text { se } \quad \varepsilon=1 .
\end{array}
$$

Vamos analisar o caso em que $\varepsilon=-1$. Primeiramente, vamos verificar que as funções $k(v)$ e $h(v)$, apresentadas no Exemplo (3.1), satisfazem (3.9). Tome $\sqrt{K(I)}=c$, assim podemos escrever

$$
\begin{aligned}
& K(I)=c^{2} \\
& k(v)=\operatorname{arcsenh}\left(\frac{1}{c} \operatorname{sen}(c v)\right) \\
& h(v)=-\sqrt{\frac{1+c^{2}}{c^{2}}} \arctan \left(\frac{\cos c v}{\sqrt{c^{2}+\operatorname{sen}^{2} c v}}\right) .
\end{aligned}
$$

Derivando $h(v)$, obtemos

$$
h^{\prime}(v)=\frac{\sqrt{c^{2}+1} \operatorname{sen} c v}{\sqrt{c^{2}+\operatorname{sen}^{2} c v}} .
$$

Tome $k(v)=\operatorname{arcsenh}(\theta(v))$, onde $\theta(v)=\frac{1}{c} \operatorname{sen}(c v)$, assim

$$
\operatorname{senh} k(v)=\theta(v)
$$

e

$$
\cosh k(v)=\sqrt{1+\theta^{2}(v)}
$$


de onde $\operatorname{coth} k(v)=\frac{\sqrt{1+\theta^{2}(v)}}{\theta(v)}=\frac{\sqrt{c^{2}+\operatorname{sen}^{2} c(v)}}{\operatorname{sen} c v}$.

Logo

$$
h^{\prime}(v) \operatorname{coth} k(v)=\frac{\sqrt{c^{2}+1} \operatorname{sen} c v}{\sqrt{c^{2}+\operatorname{sen}^{2} c v}} \frac{\sqrt{c^{2}+\operatorname{sen}^{2} c v}}{\operatorname{sen} c v}=\sqrt{c^{2}+1} .
$$

Suponha $h^{\prime}(v) \operatorname{coth} k(v)=b$. Se $b>0$, tome o parâmetro $v$. Se $b<0$ reparametrize por $-v$. Segue que as funções $h(v)$ e $k(v)$, apresentadas no exemplo (3.1), são de fato soluções de (3.9). Usando que $k^{\prime}(v)^{2}+h^{\prime}(v)^{2}=1$, as soluções para (3.9) são dadas por

$$
k(v)=k_{0}\left( \pm\left(v+c_{0}\right)\right), \quad h(v)= \pm h_{0}\left( \pm\left(v+c_{0}\right)\right)+c_{1}
$$

onde $c_{0}, c_{1}$ são duas constantes reais e $k_{0}(v), h_{0}(v)$ são aquelas do Exemplo (3.1).

O caso em que $\varepsilon=1$, é inteiramente análogo, no qual obteríamos as mesmas expressões para as soluções de (3.10), como vistas em (3.11), para $h(v)$ e $k(v)$.

Portanto, $\psi$ é, a menos de reparametrizações e translações verticais, um pedaço da superfície completa de curvatura Gaussiana constante.

Agora, estamos prontos para obter nossa estimativa da altura.

\section{Teorema 3.2.}

Seja $\psi: \Sigma \rightarrow \mathbb{M}^{2}(\varepsilon) \times \mathbb{R}$ um gráfico compacto sobre um conjunto $\Omega \subset \mathbb{M}^{2}(\varepsilon)$, com curvatura Gaussiana constante positiva $K(I)>\varepsilon$ e cuja fronteira está contida em um slice $\mathbb{M}^{2}(\varepsilon) \times\{0\}$. Então a altura máxima que $\psi$ pode atingir em $\mathbb{M}^{2}(\varepsilon) \times\{0\}$ é

$$
\begin{array}{ll}
\sqrt{\frac{K(I)+1}{K(I)}} \arctan \left(\frac{1}{\sqrt{K(I)}}\right) & \text { se } \varepsilon=-1, \\
\sqrt{\frac{K(I)-1}{K(I)}} \ln \left(\frac{\sqrt{K(I)}+1}{\sqrt{K(I)-1}}\right) & \text { se } \varepsilon=1 .
\end{array}
$$

Além disso, a igualdade ocorre se, e somente se, $\psi$ é o hemisfério de uma $K(I)$-superfície completa.

Demonstração. Observe que, como $K(I)$ é positiva e maior que $\varepsilon$, de $(2.2)$ e (2.6) a curvatura extrínseca $K$ é também positiva. Podemos assumir, sem perda de generalidade, que $\psi$ está contida sobre o slice $\mathbb{M}^{2}(\varepsilon) \times\{0\}$ e então $\nu \leq 0$ em toda parte por causa da escolha da orientação. 
Vamos considerar as funções

$$
\begin{aligned}
& f=\sqrt{\frac{K(I)+1}{K(I)}} \arctan \left(\frac{\nu}{\sqrt{K(I)}}\right), \quad \text { se } \varepsilon=-1, \\
& f=-\sqrt{\frac{K(I)-1}{K(I)}} \ln \left(\frac{\sqrt{K(I)}-\nu}{\sqrt{K(I)-\nu^{2}}}\right), \quad \text { se } \quad \varepsilon=1
\end{aligned}
$$

e $\phi=h+f$ em $\Sigma$. Observe que $\phi=f \leq 0$ sobre $\partial \Sigma$. Nosso objetivo é mostrar que $\phi_{z \bar{z}} \geq 0$ em $\Sigma$, porque sob essas condições o princípio do máximo assegura que $\phi \leq 0 \mathrm{em}$ $\Sigma$. Deste último fato nossa estimativa para o máximo da altura segue facilmente, isto é, se $\phi \leq 0$, então (3.12) e (3.13) ocorrem. Observe que esses limites são ótimos, porque eles são atingidos para o hemisfério da $K(I)$-superfícies completas.

Vamos mostrar que $\phi_{z \bar{z}} \geq 0$. De (2.20), (2.14), (2.18) e (2.28), obtemos

$$
f_{z \bar{z}}=\frac{\sqrt{K(I)-\varepsilon}}{K^{2}}\left(K \nu_{z \bar{z}}+2 \varepsilon \nu\left|\nu_{z}\right|^{2}\right)=-\frac{\sqrt{K(I)-\varepsilon} \nu}{K}\left(\frac{\varepsilon|\alpha|^{2}}{D}+K M\right) \text {. }
$$

Usando (2.22)

$$
|\alpha|^{2}=\alpha \bar{\alpha}=\left(\bar{L} h_{z}-M h_{\bar{z}}\right)\left(L h_{\bar{z}}-M h_{z}\right)=\left(|L|^{2}+M^{2}\right)\left|h_{z}\right|^{2}-M\left(\bar{L} h_{z}^{2}+L h_{\bar{z}}^{2}\right) .
$$

Já verificamos que

$$
\|T\|^{2}=\frac{1}{D}\left(\alpha h_{z}+\bar{\alpha} h_{\bar{z}}\right) .
$$

Novamente usando (2.22), obtemos

$$
\|T\|^{2}=\frac{1}{D}\left(\bar{L} h_{z}^{2}+L h_{\bar{z}}^{2}-2 M\left|h_{z}\right|^{2}\right) .
$$

De $(3.14)$ e (3.15),

$$
\begin{aligned}
\frac{|\alpha|^{2}}{D} & =\frac{|L|^{2}+M^{2}}{D}\left|h_{z}\right|^{2}-M \frac{1}{D}\left(\bar{L} h_{z}^{2}+L h_{\bar{z}}^{2}\right) \\
& =\frac{|L|^{2}-M^{2}}{D}\left|h_{z}\right|^{2}-M \frac{1}{D}\left(\bar{L} h_{z}^{2}+L h_{\bar{z}}^{2}-2 M\left|h_{z}\right|^{2}\right) \\
& =\left|h_{z}\right|^{2}-M\|T\|^{2} .
\end{aligned}
$$

Usando (2.14)

$$
\begin{aligned}
K M+\frac{\varepsilon|\alpha|^{2}}{D} & =K M+\varepsilon\left(\left|h_{z}\right|^{2}-M\|T\|^{2}\right) \\
& =M\left(K-\varepsilon\|T\|^{2}\right)+\varepsilon\left|h_{z}\right|^{2} \\
& =M\left(K-\varepsilon\left(1-\nu^{2}\right)\right)+\varepsilon\left|h_{z}\right|^{2} \\
& =M(K(I)-\varepsilon)+\varepsilon\left|h_{z}\right|^{2} .
\end{aligned}
$$


Assim, usando (2.27) ficamos com

$$
\phi_{z \bar{z}}=\frac{(K(I)-\varepsilon) \nu}{K}\left(\rho-\sqrt{K(I)-\varepsilon}\left(M+\frac{\varepsilon\left|h_{z}\right|^{2}}{K(I)-\varepsilon}\right)\right) .
$$

De [2], Lema 1, temos

$$
K(I)-\varepsilon=\frac{\rho^{2}}{\left(M+\frac{\varepsilon\left|h_{z}\right|^{2}}{K(I)-\varepsilon}\right)^{2}-\frac{|Q|^{2}}{(K(I)-\varepsilon)^{2}}},
$$

ou equivalentemente

$$
\rho^{2}-(K(I)-\varepsilon)\left(M+\frac{\varepsilon\left|h_{z}\right|^{2}}{K(I)-\varepsilon}\right)^{2}=-\frac{|Q|^{2}}{K(I)-\varepsilon} .
$$

Já que $M+\frac{\varepsilon\left|h_{z}\right|^{2}}{K(I)-\varepsilon}$ é a $(1,1)$-parte da métrica $A$ com respeito a $I I$, então essa expressão deve ser positiva. Portanto, de (3.16) e (3.17)

$$
\begin{aligned}
\phi_{z \bar{z}} & =\frac{(K(I)-\varepsilon) \nu}{K}\left[-\frac{Q^{2}}{(K(I)-\varepsilon)(\rho+\sqrt{K(I)-\varepsilon})\left(M+\frac{\varepsilon\left|h_{z}\right|^{2}}{K(I)-\varepsilon}\right)}\right] \\
& =-\frac{\nu}{K} \frac{Q^{2}}{(\rho+\sqrt{K(I)-\varepsilon})\left(M+\frac{\varepsilon\left|h_{z}\right|^{2}}{K(I)-\varepsilon}\right)} \geq 0 .
\end{aligned}
$$

Finalmente, note que se a altura máxima é atingida, então $\phi_{z \bar{z}} \equiv 0$. Isto é, $Q$ ou $\nu$ é identicamente nulo em $\Sigma$. Mas, $\psi$ não pode estar contida em um cilindro porque $K(I) \neq 0$, isto é, $\nu \not \equiv 0$. Portanto, $Q \equiv 0$ e o resultado segue do Teorema (3.1).

Observação 3.1. Do Teorema de Bonnet-Myers, pode-se deduzir que toda geodésica minimizante em uma superfície $\psi: \Sigma \rightarrow \mathbb{M}^{2}(\varepsilon) \times \mathbb{R}$ com curvatura Gaussiana positiva $K(I)$ tem comprimento menor ou igual a $\frac{2 \pi}{\sqrt{K(I)}}$. Consequentemente, sempre existem estimativas de altura para tais superfícies, embora essas estimativas estão longe de serem ótimas. Os limites que nós fornecemos no teorema acima é, como vimos, ótimos.

Gostaríamos de salientar que a hipótese $K(I) \geq 1$ em $\mathbb{S}^{2} \times \mathbb{R}$ pode ser mudada para $K(I)>0, K(I) \neq 1$. De fato, se $\psi: \Sigma \rightarrow \mathbb{M}^{2}(\varepsilon) \times \mathbb{R}$ é uma superfície com curvatura Gaussiana $K(I), 0<K(I)<1$, então lá não existe um ponto $p$ onde a função altura atinge um máximo ou mínimo, porque da equação de Gauss seria $k_{1}(p) k_{2}(p)<0$ e assim em qualquer vizinhança de $p$ haveria pontos de maior ou menor altura que $p$. Em particular, respondemos a questão posta pelos autores em [2]. 


\section{Proposição 3.2 .}

Não existe uma superfície completa com curvatura Gaussiana constante $K(I), 0<K(I)<$ $1, e m \mathbb{S}^{2} \times \mathbb{R}$. 


\section{Representação}

Neste capítulo, veremos o teorema de representação para superfícies de curvatura Gaussiana constante, que nos permite escrever as primeira e segunda formas fundamentais em termos da função altura $h$, a quarta coordenada do seu normal unitário $\nu$ e da constante $K(I)$. Damos especial atenção aos casos particulares: $K(I)=1 \mathrm{em} \mathbb{S}^{2} \times \mathbb{R}$ e $K(I)=-1$ em $\mathbb{H}^{2} \times \mathbb{R}$.

\subsection{Representação de $K(I)$-superfícies com $K>0$}

Seja $\psi: \Sigma \rightarrow \mathbb{M}^{2}(\varepsilon) \times \mathbb{R}$ uma $K(I)$-superfície com curvatura extrínseca positiva e assuma que $I I$ é positiva definida. Também assumiremos que a superfície é transversa para cada slice $\mathbb{M}^{2}(\varepsilon) \times\{t\}$ para todo $t \in \mathbb{R}$, isto é, $T$ nunca se anula em $\Sigma$, ou equivalentemente, $\nu^{2}<1$ em $\Sigma$. Nos referiremos a tais superfícies como superfícies transversas.

Vamos observar que os pontos onde $\nu^{2}=1$ é um conjunto isolado porque $K<0$ (isto é, $I I$ é positiva definida).

Segue de (2.22)

$$
\bar{L}=\frac{\alpha+M h_{\bar{z}}}{h_{z}}
$$

e de (2.19) temos

$$
\frac{\alpha h_{z}+\bar{\alpha} h_{\bar{z}}}{1-\nu^{2}}=D=|L|^{2}-M^{2}=\left|\frac{\alpha+M h_{\bar{z}}}{h_{z}}\right|^{2}-M^{2},
$$

isto é,

$$
M=-\frac{|\alpha|^{2}}{\alpha h_{z}+\bar{\alpha} h_{\bar{z}}}+\frac{\left|h_{z}\right|^{2}}{1-\nu^{2}} .
$$

Consequentemente, usando (4.1)

$$
L=\frac{\bar{\alpha}^{2}}{\alpha h_{z}+\bar{\alpha} h_{\bar{z}}}+\frac{h_{z}^{2}}{1-\nu^{2}} .
$$

Por outro lado, de (2.14) e (2.18) obtemos

$$
\alpha=\frac{\rho \nu_{\bar{z}}}{K(I)-\varepsilon \nu^{2}} .
$$


Além disso, de (2.20), (2.14) e (2.19)

$$
\rho^{2}=-D K=-\frac{K(I)-\varepsilon \nu^{2}}{1-\nu^{2}}\left(\alpha h_{z}+\bar{\alpha} h_{\bar{z}}\right)
$$

De $(4.2)$ e $(4.3)$

$$
\bar{\alpha}^{2}=-\frac{\nu_{z}^{2}}{\left(1-\nu^{2}\right)\left(K(I)-\varepsilon \nu^{2}\right)}\left(\alpha h_{z}+\bar{\alpha} h_{\bar{z}}\right) .
$$

Com tudo isto, $L, M$ podem ser escritos em termos de $h_{z}, \nu, \nu_{z}$ e $K(I)$ como

$$
\begin{aligned}
L & =\frac{h_{z}^{2}}{1-\nu^{2}}-\frac{\nu_{z}^{2}}{\left(1-\nu^{2}\right)\left(K(I)-\varepsilon \nu^{2}\right)}, \\
M & =\frac{\left|h_{z}\right|^{2}}{1-\nu^{2}}+\frac{\left|\nu_{z}\right|^{2}}{\left(1-\nu^{2}\right)\left(K(I)-\varepsilon \nu^{2}\right)} .
\end{aligned}
$$

Assim, substituindo as expressões acima encontradas para $L$ e $M$ em (2.22), obtemos

$$
\alpha=-\frac{\nu_{z} h_{\bar{z}}+\nu_{\bar{z}} h_{z}}{\left(1-\nu^{2}\right)\left(K(I)-\varepsilon \nu^{2}\right)} \nu_{\bar{z}}
$$

e de $(4.2)$

$$
\rho=-\frac{\nu_{z} h_{\bar{z}}+\nu_{\bar{z}} h_{z}}{1-\nu^{2}}>0
$$

Neste ponto pode ser interessante observar que a métrica induzida e a segunda forma fundamental podem ser recuperadas para qualquer superfície transversa com curvatura extrínseca positiva em termos de $h, \nu, K(I)$ e da estrutura conforme dada por $I I$. De fato, as equações de compatibilidade para a existência de uma imersão de uma superfície Riemanniana simplesmente conexa com funções fixadas $h: \Sigma \rightarrow \mathbb{R}$ e $\nu: \Sigma \rightarrow(-1,1)$ e curvatura Gaussiana fixada são dadas pelo Lema (2.1).

As equações de compatibilidade são muito mais simples para uma superfície de curvatura Gaussiana constante:

\section{Teorema 4.1.}

Sejam $\Sigma$ uma superfície e $\psi: \Sigma \rightarrow \mathbb{M}^{2}(\varepsilon) \times \mathbb{R}$ uma $K(I)$-imersão transversa tal que $K>0$. Então, dada um parâmetro local conforme z para II, a primeira e segunda formas fundamentais de $\psi$ podem ser recuperadas em termos de sua função altura $h, \nu e$ 
a constante $K(I)$ como

$$
\begin{aligned}
& L=\frac{h_{z}^{2}}{1-\nu^{2}}-\frac{\nu_{z}^{2}}{\left(1-\nu^{2}\right)\left(K(I)-\varepsilon \nu^{2}\right)}, \\
& M=\frac{\left|h_{z}\right|^{2}}{1-\nu^{2}}+\frac{\left|\nu_{z}\right|^{2}}{\left(1-\nu^{2}\right)\left(K(I)-\varepsilon \nu^{2}\right)}, \\
& \rho=-\frac{\nu_{z} h_{\bar{z}}+\nu_{\bar{z}} h_{z}}{1-\nu^{2}}>0 .
\end{aligned}
$$

Além disso $h$ e $\nu$ satisfazem

$$
\begin{gathered}
h_{z \bar{z}}=-\frac{(K(I)-\varepsilon) \nu}{\left(1-\nu^{2}\right)\left(K(I)-\varepsilon \nu^{2}\right)}\left(\nu_{z} h_{\bar{z}}+\nu_{\bar{z}} h_{z}\right), \\
\nu_{z \bar{z}}=-\frac{\nu}{\left(1-\nu^{2}\right)\left(K(I)-\varepsilon \nu^{2}\right)}\left(\left(K(I)-2 \varepsilon \nu^{2}+\varepsilon\right)\left|\nu_{z}\right|^{2}\right. \\
\left.\quad+\left(K(I)-\varepsilon \nu^{2}\right)^{2}\left|h_{z}\right|^{2}\right) .
\end{gathered}
$$

Reciprocamente, sejam $K(I) \in \mathbb{R}$ e $\varepsilon \in\{-1,1\}$ uma constante, $h: \Sigma \rightarrow \mathbb{R}$ e $\nu: \Sigma \rightarrow(-1,1)$ funções sobre uma superfície de Riemann $\Sigma$ simplesmente conexa satisfazendo (4.8) , (4.9), $K(I)-\varepsilon \nu^{2}>0$ e $\nu_{z} h_{\bar{z}}+\nu_{\bar{z}} h_{z}<0$. Então, existe uma imersão $\psi: \Sigma \rightarrow \mathbb{M}^{2}(\varepsilon) \times \mathbb{R}$ tal que $\Sigma$ é uma $K(I)$-superfície transversa cuja primeira e segunda formas são dadas por

$$
\begin{aligned}
I & =L d z^{2}+2 M|d z|^{2}+\bar{L} d \bar{z}^{2}, \\
I I & =2 \rho|d z|^{2},
\end{aligned}
$$

onde L, M e $\rho$ são definidas por (4.5), (4.6) e (4.7), respectivamente; cuja função altura e a quarta coordenada de seu normal são dadas por $h$ e $\nu$, respectivamente; e a estrutura dada por sua segunda forma fundamental é a de $\Sigma$. Além disso, essa imersão é única a menos de isometria de $\mathbb{M}^{2}(\varepsilon) \times \mathbb{R}$.

Demonstração. Para a primeira parte observamos que (4.5), (4.6) e (4.7) já foram calculados. Além disso, (4.8) e (4.9) são equivalentes a (2.27) e (2.28) quando as expressões anteriores de $L, M$ e $\rho$ são usadas.

Para a segunda parte, usando (4.5), (4.6) e (4.7), devemos verificar que as equações (2.14) - (2.19) do Lema (2.1) são satisfeitas. 
Por um cálculo direto obtemos

$$
\begin{aligned}
|L|^{2} & =\frac{1}{\left(1-\nu^{2}\right)^{2}}\left[\left|h_{z}\right|^{4}-\frac{\nu_{\bar{z}} h_{z}^{2}+\nu_{z}^{2} h_{\bar{z}}^{2}}{K(I)-\varepsilon \nu^{2}}+\frac{\left|\nu_{z}\right|^{4}}{\left(K(I)-\varepsilon \nu^{2}\right)^{2}}\right], \\
M^{2} & =\frac{1}{\left(1-\nu^{2}\right)^{2}}\left[\left|h_{z}\right|^{4}+2 \frac{\left|h_{z}\right|^{2}\left|\nu_{z}\right|^{2}}{K(I)-\varepsilon \nu^{2}}+\frac{\left|\nu_{z}\right|^{4}}{\left(K(I)-\varepsilon \nu^{2}\right)^{2}}\right], \\
\rho^{2} & =\left(\frac{h_{\bar{z}} \nu_{z}+\nu_{\bar{z}} h_{z}}{1-\nu^{2}}\right)^{2} .
\end{aligned}
$$

De onde

$$
D=|L|^{2}-M^{2}=-\frac{\left(\nu_{\bar{z}} h_{z}+\nu_{z} h_{\bar{z}}\right)^{2}}{\left(1-\nu^{2}\right)^{2}\left(K(I)-\varepsilon \nu^{2}\right)} .
$$

De (2.20), (4.10) e (4.11) obtemos (2.14).

Resulta de (4.4)

$$
\alpha h_{z}+\bar{\alpha} h_{\bar{z}}=-\frac{1}{\left(1-\nu^{2}\right)\left(K(I)-\varepsilon \nu^{2}\right)}\left(\nu_{\bar{z}} h_{z}+\nu_{z} h_{\bar{z}}\right)^{2} .
$$

De (4.11) e (4.12) obtemos (2.19).

Verificamos (2.18) usando (2.20), (4.4) e (4.11).

Nesta parte, será necessário calcularmos os símbolos de Christoffel. De (4.5), (4.8) e (4.9)

$$
\begin{aligned}
& L_{\bar{z}}=-\frac{2 \varepsilon \nu h_{\bar{z}} \rho}{K}=\frac{2 \varepsilon \nu h_{z}\left(\nu_{z} h_{\bar{z}}+\nu_{\bar{z}} h_{z}\right)}{\left(1-\nu^{2}\right)\left(K(I)-\varepsilon \nu^{2}\right)}, \\
& \bar{L}_{z}=-\frac{2 \varepsilon \nu h_{z} \rho}{K}=\frac{2 \varepsilon \nu h_{\bar{z}}\left(\nu_{z} h_{\bar{z}}+\nu_{\bar{z}} h_{z}\right)}{\left(1-\nu^{2}\right)\left(K(I)-\varepsilon \nu^{2}\right)},
\end{aligned}
$$

segue que

$$
\bar{L} L_{\bar{z}}-M \bar{L}_{z}=-\frac{2 \varepsilon\left(\nu_{z} h_{\bar{z}}+\nu_{\bar{z}} h_{z}\right)^{2} \nu \nu_{\bar{z}}}{\left(1-\nu^{2}\right)^{2}\left(K(I)-\varepsilon \nu^{2}\right)^{2}} .
$$

Sabemos que

$$
\Gamma_{12}^{1}=\frac{1}{2 D}\left(\bar{L} L_{\bar{z}}-M \bar{L}_{z}\right) .
$$

Usando (4.11) e (4.13), obtemos

$$
\Gamma_{12}^{1}=\frac{\varepsilon \nu \nu_{\bar{z}}}{K(I)-\varepsilon \nu^{2}} .
$$

Decorre de $\Gamma_{12}^{2}=\overline{\Gamma_{12}^{1}}$ que

$$
\Gamma_{12}^{2}=\frac{\varepsilon \nu \nu_{z}}{K(I)-\varepsilon \nu^{2}} .
$$


Note que

$$
\Gamma_{12}^{1}=\frac{\varepsilon \alpha \nu}{\rho} .
$$

Usando (4.7), (4.14), (4.15) podemos verificar que (2.17) é satisfeita.

Como

$$
\Gamma_{12}^{1}+\Gamma_{22}^{2}=\frac{D_{\bar{z}}}{2 D}
$$

segue que

$$
\Gamma_{12}^{1}-\Gamma_{22}^{2}=2 \Gamma_{12}^{1}-\frac{D_{\bar{z}}}{2 D}
$$

Usando (2.30), obtemos

$$
\Gamma_{12}^{1}-\Gamma_{22}^{2}=2 \Gamma_{12}^{1}-\frac{\rho_{\bar{z}}}{\rho}+\frac{K_{\bar{z}}}{2 K}
$$

Portanto

$$
\frac{\rho_{\bar{z}}}{\rho}+\Gamma_{12}^{1}-\Gamma_{22}^{2}=2 \Gamma_{12}^{1}+\frac{K_{\bar{z}}}{2 K}
$$

De (2.32), (4.16) e (4.17) segue que

$$
\frac{\rho_{\bar{z}}}{\rho}+\Gamma_{12}^{1}-\Gamma_{22}^{2}=\frac{\varepsilon \alpha \nu}{\rho} .
$$

E está provado (2.15). Resta, por fim, demonstrarmos a equação (2.16). Sabemos que

$$
\Gamma_{12}^{2}+\Gamma_{11}^{1}=\frac{D_{z}}{2 D}
$$

assim

$$
\Gamma_{11}^{1}=\frac{D_{z}}{2 D}-\Gamma_{12}^{2}=\frac{D_{z}}{2 D}-\overline{\Gamma_{12}^{1}}
$$

Novamente, usando (2.30), (2.32), concluímos que

$$
\frac{D_{z}}{2 D}=\frac{\rho_{z}}{\rho}+\frac{\varepsilon \nu \bar{\alpha}}{\rho} .
$$

Logo de (4.16), (4.18) e (4.19), segue que

$$
\Gamma_{11}^{1}=\frac{\rho_{z}}{\rho}+\frac{\varepsilon \nu \bar{\alpha}}{\rho}-\frac{\varepsilon \nu \bar{\alpha}}{\rho},
$$

isto é,

$$
\Gamma_{11}^{1}=\frac{\rho_{z}}{\rho}
$$


Sabemos que

$$
\begin{aligned}
& \Gamma_{11}^{1}=\frac{1}{2 D}\left(\bar{L} L_{z}-2 M M_{z}+M L_{\bar{z}}\right) \\
& \Gamma_{11}^{2}=-\frac{1}{2 D}\left(L L_{\bar{z}}-2 L M_{z}+M L_{z}\right),
\end{aligned}
$$

então

$$
\Gamma_{11}^{1} h_{z}+\Gamma_{11}^{2} h_{\bar{z}}=\frac{1}{2 D}\left[L_{z}\left(\bar{L} h_{z}-M h_{\bar{z}}\right)+\left(L_{\bar{z}}-2 M_{z}\right)\left(M h_{z}-L h_{\bar{z}}\right)\right]
$$

De (4.5) e (4.6)

$$
\begin{aligned}
L h_{z}-M h_{\bar{z}} & =\frac{1}{1-\nu^{2}}\left[h_{\bar{z}}^{2} h_{z}-\frac{\nu_{\bar{z}}^{2} h_{z}}{K}-h_{z} h_{\bar{z}}^{2}-\frac{\nu_{z} \nu_{\bar{z}} h_{\bar{z}}}{K}\right] \\
& =-\frac{\nu_{\bar{z}}}{K\left(1-\nu^{2}\right)}\left(\nu_{\bar{z}} h_{z}+\nu_{z} h_{\bar{z}}\right) \\
& =\alpha
\end{aligned}
$$

e

$$
\begin{aligned}
M h_{z}-L h_{\bar{z}} & =\frac{1}{1-\nu^{2}}\left[h_{z}^{2} h_{\bar{z}}+\frac{\nu_{z} \nu_{\bar{z}} h_{z}}{K}-h_{z}^{2} h_{\bar{z}}+\frac{\nu_{z}^{2} h_{\bar{z}}}{K}\right] \\
& =\frac{\nu_{z}}{K\left(1-\nu^{2}\right)}\left(\nu_{\bar{z}} h_{z}+\nu_{z} h_{\bar{z}}\right) \\
& =-\bar{\alpha} .
\end{aligned}
$$

E concluímos de (4.21) que

$$
\Gamma_{11}^{1} h_{z}+\Gamma_{11}^{2} h_{\bar{z}}=\frac{1}{2 D}\left[L_{z} \alpha-\left(L_{\bar{z}}-2 M_{z}\right) \bar{\alpha}\right] .
$$

Mas $\alpha=\frac{\rho}{K} \nu_{\bar{z}}, \log 0$ usando $(2.20)$

$$
\Gamma_{11}^{1} h_{z}+\Gamma_{11}^{2} h_{\bar{z}}=-\frac{1}{2 \rho}\left[L_{z} \nu_{\bar{z}}-L_{\bar{z}} \nu_{z}+2 M_{z} \nu_{z}\right]
$$

Por sua vez

$$
\begin{aligned}
L_{z} & =\frac{2}{1-\nu^{2}}\left[L \nu \nu_{z}+h_{z} h_{z z}-\frac{\nu_{z} \nu_{z z}}{K}-\frac{2 \varepsilon \nu \nu_{z}^{3}}{K^{2}}\right] \\
L_{\bar{z}} & =\frac{2}{1-\nu^{2}}\left[L \nu \nu_{\bar{z}}+h_{z} h_{z \bar{z}}-\frac{\nu_{z} \nu_{z \bar{z}}}{K}-\frac{2 \varepsilon \nu \nu_{z}^{2} \nu_{\bar{z}}}{K^{2}}\right], \\
M_{z} & =\frac{1}{1-\nu^{2}}\left[2 M \nu \nu_{z}+h_{z z} h_{\bar{z}}+h_{z} h_{z \bar{z}}+\frac{\nu_{z z} \nu_{\bar{z}}+\nu_{z} \nu_{z \bar{z}}}{K}+\frac{2 \varepsilon \nu \nu_{z}^{2} \nu_{\bar{z}}}{K^{2}}\right],
\end{aligned}
$$

assim

$$
L_{z} \nu_{\bar{z}}-L_{\bar{z}} \nu_{z}=\frac{2}{1-\nu^{2}}\left[h_{z}\left(h_{z z} \nu_{\bar{z}}-h_{z \bar{z}} \nu_{z}\right)-\frac{\nu_{z}\left(\nu_{z z} \nu_{\bar{z}}-\nu_{z \bar{z}} \nu_{\bar{z}}\right)}{K}\right]
$$


$\mathrm{e}$

$$
\begin{aligned}
L_{z} \nu_{\bar{z}}-L_{\bar{z}} \nu_{z}+2 M_{z} \nu_{z}= & \frac{1}{1-\nu^{2}}\left[2 h_{z}\left(h_{z z} \nu_{\bar{z}}-h_{z \bar{z}} \nu_{z}\right)-\frac{2 \nu_{z}\left(\nu_{z z} \nu_{\bar{z}}-\nu_{z \bar{z}} \nu_{\bar{z}}\right)}{K}+4 M \nu \nu_{z}^{2}\right. \\
& \left.+2 h_{z z} h_{\bar{z}} \nu_{z}+2 h_{z} h_{z \bar{z}} \nu_{z}+\frac{2 \nu_{z}\left(\nu_{z z} \nu_{\bar{z}}+\nu_{z} \nu_{z \bar{z}}\right)}{K}+\frac{4 \varepsilon \nu \nu_{z}^{2}\left|\nu_{z}\right|^{2}}{K^{2}}\right] \\
= & \frac{1}{1-\nu^{2}}\left[2 h_{z z}\left(h_{z} \nu_{\bar{z}}+h_{\bar{z}} \nu_{z}\right)+\frac{4 \nu_{z}^{2} \nu_{z \bar{z}}}{K}+4 M \nu \nu_{z}^{2}+\frac{4 \varepsilon \nu \nu_{z}^{2}\left|\nu_{z}\right|^{2}}{K^{2}}\right] \\
= & -2 h_{z z} \rho+\frac{4 \nu_{z}^{3}}{1-\nu^{2}}\left[-\frac{\nu}{K^{2}\left(1-\nu^{2}\right)}\left(\left(K(I)-2 \varepsilon \nu^{2}+\varepsilon\right)\left|\nu_{z}\right|^{2}\right.\right. \\
& \left.\left.+K^{2}\left|h_{z}\right|^{2}\right)+\frac{\nu}{1-\nu^{2}}\left(\left|h_{z}\right|^{2}+\frac{\left|\nu_{z}\right|^{2}}{K}\right)+\frac{\varepsilon \nu\left|\nu_{z}\right|^{2}}{K\left(1-\nu^{2}\right)}\right] \\
= & -2 h_{z z} \rho+\frac{4 \nu_{z}^{3}}{1-\nu^{2}}\left[-\frac{\nu}{K^{2}\left(1-\nu^{2}\right)}\left(K-\varepsilon\left(\nu^{2}-1\right)\right)\left|\nu_{z}\right|^{2}\right. \\
& \left.+\frac{\nu\left|\nu_{z}\right|^{2}}{K\left(1-\nu^{2}\right)}+\frac{\varepsilon \nu\left|\nu_{z}\right|^{2}}{K^{2}}\right] \\
= & -2 h_{z z} \rho,
\end{aligned}
$$

$\log 0$

$$
\Gamma_{11}^{1} h_{z}+\Gamma_{11}^{2} h_{\bar{z}}=-\frac{1}{2 \rho}\left(-2 h_{z z} \rho\right)=h_{z z}
$$

Essa representação tem especial interesse para superfícies transversas com curvatura Gaussiana constante $K(I)=1$ em $\mathbb{S}^{2} \times \mathbb{R}$. Nesse caso, os slices são as únicas superfícies completas com curvatura Gaussiana constante $K(I)=1$ em $\mathbb{S}^{2} \times \mathbb{R}$ (veja [2]). Além disso, as equações de compatibilidade podem ser reduzidas a equação sinh-Gordon elípticas.

Assim, seja $\psi: \Sigma \rightarrow \mathbb{S}^{2} \times \mathbb{R}$ uma imersão transversa com $K(I)=1$ nas condições anteriores. Já que $\nu^{2}<1$, segue de (2.14) que $K>0$ e então podemos assumir que $I I$ é uma métrica Riemanniana em $\Sigma$. Portanto, estamos sob as hipóteses do Teorema 4.1 e consequentemente a função altura $h$ é harmônica para $I I$ e $\nu$ satisfaz

$$
\nu_{z \bar{z}}=-\nu\left(\left|h_{z}\right|^{2}+2 \frac{\left|\nu_{z}\right|^{2}}{1-\nu^{2}}\right) .
$$

Se definirmos $\omega=\operatorname{arctanh}(\nu)$, esta última equação torna-se

$$
\omega_{z \bar{z}}+\left|h_{z}\right|^{2} \operatorname{senh}(\omega) \cosh (\omega)=0
$$

e podemos reformular o Teorema 4.1 para essas superfícies como segue: 


\section{Corolário 4.1.}

Sejam $\Sigma$ uma superfície conexa e $\psi: \Sigma \rightarrow \mathbb{S}^{2} \times \mathbb{R}$ uma imersão transversa com $K(I)=$ 1. Então, dado um parâmetro conforme local z para II, a primeira e segunda formas fundamentais de $\psi$ podem ser recuperadas em termos de sua função altura $h$ e $\omega=$ $\operatorname{arctanh}(\nu)$ como

$$
\begin{aligned}
& L=\cosh ^{2}(\omega) h_{z}^{2}-\omega_{z}^{2}, \\
& M=\cosh ^{2}(\omega)\left|h_{z}\right|^{2}+\left|\omega_{z}\right|^{2}, \\
& \rho=-\left(\omega_{z} h_{\bar{z}}+\omega_{\bar{z}} h_{z}\right)>0,
\end{aligned}
$$

onde $\omega$ satisfaz (4.22).

Reciprocamente, sejam $h: \Sigma \rightarrow \mathbb{R}$ uma função harmônica sobre uma superfície Riemanniana simplesmente conexa $\Sigma e \omega: \Sigma \rightarrow \mathbb{R}$ uma função satisfazendo $\omega_{z} h_{\bar{z}}+\omega_{\bar{z}} h_{z}<0$ $e$ (4.22). Então, existe uma imersão $\psi: \Sigma \rightarrow \mathbb{S}^{2} \times \mathbb{R}$ tal que $\Sigma$ é transversa com curvatura Gaussiana constante $K(I)=1$, cuja primeira e segunda formas fundamentais são dadas por

$$
\begin{aligned}
I & =L d z^{2}+2 M|d z|^{2}+\bar{L} d \bar{z}^{2}, \\
I I & =2 \rho|d z|^{2},
\end{aligned}
$$

onde L, M e $\rho$ são definidas por (4.23), (4.24) e (4.25) respectivamente, cuja função altura e quarta coordenada de seu normal são dadas por $h$ e $\nu=\tanh (\omega)$, respectivamente; e a estrutura dada por sua segunda forma fundamental é a de $\Sigma$. Além disso, essa imersão é única a menos de isometria de $\mathbb{S}^{2} \times \mathbb{R}$.

Já que $h_{z} \neq 0$ em uma imersão transversa e $h_{z}$ é uma função holomorfa quando $K(I)=1$ em $\mathbb{S}^{2} \times \mathbb{R}$, podemos tomar um parâmetro conforme $\zeta$ tal que $d \zeta=h_{z} d z$. Assim, (4.22) torna-se a clássica equação sinh-Gordon elíptica

$$
\omega_{\zeta \bar{\zeta}}+\operatorname{senh}(\omega) \cosh (\omega)=0
$$

\subsection{Representação de $K(I)$-superfícies com $K<0$}

Resultados similares àqueles apresentados na seção anterior podem ser obtidos para $K(I)$-superfícies transversas com curvatura extrínseca negativa. Neste caso, $I I$ é uma 
métrica Lorentziana e $\Sigma$ pode ser considerada uma superfície de Lorentz com estrutura conforme induzida.

Então, podemos tomar coordenadas assintóticas $(u, v)$ para a superfície com $K<0$, tal que a primeira e segunda formas podem ser escritas como

$$
\begin{aligned}
& I=E d u^{2}+2 F d u d v+G d v^{2}, \\
& I I=2 f d u d v .
\end{aligned}
$$

Após um desenvolvimento similar em essência aquele do caso de curvatura extrínseca positiva obtemos alguns resultados que são enunciados sem uma demonstração a fim de não repetir os mesmos cálculos.

\section{Teorema 4.2.}

Sejam $\Sigma$ uma superfície conexa e $\psi: \Sigma \rightarrow \mathbb{M}^{2}(\varepsilon) \times \mathbb{R}$ uma $K(I)$-imersão transversa tal que $K<0$. Então, dadas coordenadas assintóticas $(u, v)$ para $I I$, a primeira e segunda formas fundamentais de $\psi$ podem ser recuperadas em termos de sua função altura $h, \nu$ e a constante $K(I)$ como

$$
\begin{aligned}
E & =\frac{h_{u}^{2}}{1-\nu^{2}}-\frac{\nu_{u}^{2}}{\left(1-\nu^{2}\right)\left(K(I)-\varepsilon \nu^{2}\right)}, \\
F & =\frac{h_{u} h_{v}}{1-\nu^{2}}+\frac{\nu_{u} \nu_{v}}{\left(1-\nu^{2}\right)\left(K(I)-\varepsilon \nu^{2}\right)}, \\
G & =\frac{h_{v}^{2}}{1-\nu^{2}}-\frac{\nu_{v}^{2}}{\left(1-\nu^{2}\right)\left(K(I)-\varepsilon \nu^{2}\right)}, \\
f & =-\frac{\nu_{u} h_{v}+\nu_{v} h_{u}}{1-\nu^{2}}>0 .
\end{aligned}
$$

Além disso, $h$ e $\nu$ satisfazem

$$
\begin{gathered}
h_{u v}=-\frac{(K(I)-\varepsilon) \nu}{\left(1-\nu^{2}\right)\left(K(I)-\varepsilon \nu^{2}\right)}\left(\nu_{u} h_{v}+\nu_{v} h_{u}\right) \\
\nu_{u v}=-\frac{\nu}{\left(1-\nu^{2}\right)\left(K(I)-\varepsilon \nu^{2}\right)}\left(\left(K(I)-2 \varepsilon \nu^{2}+\varepsilon\right) \nu_{u} \nu_{v}\right. \\
\left.\quad+\left(K(I)-\varepsilon \nu^{2}\right)^{2} h_{u} h_{v}\right) .
\end{gathered}
$$

Reciprocamente, sejam $K(I) \in \mathbb{R} e \varepsilon \in\{-1,1\}$ constantes, $h: \Sigma \rightarrow \mathbb{R}$ e $\nu: \Sigma \rightarrow(-1,1)$ funções sobre uma superfície de Lorentz simplesmente conexa $\Sigma$ satisfazendo (4.30), (4.31), $K(I)-\varepsilon \nu^{2}<0$ e $\nu_{u} h_{v}+\nu_{v} h_{u}<0$. Então existe uma imersão $\psi: \Sigma \rightarrow \mathbb{M}^{2}(\varepsilon) \times \mathbb{R}$ tal que $\Sigma$ é uma $K(I)$-superfície transversa cuja primeira e segunda 
formas fundamentais são dadas por

$$
\begin{aligned}
I & =E d u^{2}+2 F d u d v+G d v^{2}, \\
I I & =2 f d u d v
\end{aligned}
$$

onde E, F, G e f são definidas por (4.26), (4.27), (4.28) e (4.29), respectivamente; cuja função altura e a quarta coordenada de seu normal são dadas porh e $\nu$, respectivamente; e a estrutura dada por sua segunda forma fundamental é a de $\Sigma$. Além disso, essa imersão é única a menos de isometrias de $\mathbb{M}^{2}(\varepsilon) \times \mathbb{R}$.

Se $\psi: \Sigma \rightarrow \mathbb{H}^{2} \times \mathbb{R}$ é uma imersão transversa com $K(I)=-1$, segue de $(2.14)$ que $K<0$ em todo ponto. Adicionalmente, do Teorema 4.2, $h$ é harmônica para $I I$ e $\nu$ satisfaz

$$
\nu_{u v}=\nu\left(h_{u} h_{v}-2 \frac{\nu_{u} \nu_{v}}{1-\nu^{2}}\right)
$$

Por tomar $\omega=\operatorname{arctanh}(\nu)$, esta última equação torna-se

$$
\omega_{u v}-h_{u} h_{v} \operatorname{senh}(\omega) \cosh (\omega)=0 .
$$

\section{Corolário 4.2.}

Seja $\Sigma$ uma superfície conexa e $\psi: \Sigma \rightarrow \mathbb{H}^{2} \times \mathbb{R}$ uma imersão transversa com $K(I)=-1$. Então, dadas coordenadas locais assintóticas $(u, v)$ para II, a primeira e segunda formas fundamentais de $\psi$ podem ser recuperadas em temos de sua função altura $h$ e $\omega=\operatorname{arctanh}(\nu)$ como

$$
\begin{aligned}
& E=\cosh ^{2}(\omega) h_{u}^{2}+\omega_{u}^{2}, \\
& F=\cosh ^{2}(\omega) h_{u} h_{v}-\omega_{u} \omega_{v}, \\
& G=\cosh ^{2}(\omega) h_{v}^{2}+\omega_{v}^{2}, \\
& f=-\left(\omega_{u} h_{v}+\omega_{v} h_{u}\right)>0,
\end{aligned}
$$

onde $\omega$ satisfaz (4.32).

Reciprocamente, sejam $h: \Sigma \rightarrow \mathbb{R}$ uma função harmônica sobre uma superfície de Lorentz simplesmente conexa $\Sigma e \omega: \Sigma \rightarrow \mathbb{R}$ uma função satisfazendo $\omega_{u} h_{v}+\omega_{v} h_{u}<0$ e (4.32). Então, existe uma imersão $\psi: \Sigma \rightarrow \mathbb{H}^{2} \times \mathbb{R}$ tal que $\Sigma$ é transversa com curvatura Gaussiana constante $K(I)=-1$, cuja primeira e segunda formas fundamentais são dadas 
por

$$
\begin{aligned}
I & =E d u^{2}+2 F d u d v+G d v^{2}, \\
I I & =2 f d u d v,
\end{aligned}
$$

onde $E, F, G$ e $f$ são definidas por (4.33), (4.34), (4.35) e (4.36), respectivamente; cuja função altura e a quarta coordenada do seu normal são dadas por $h$ e $\nu=\tanh (\omega)$, respectivamente; e a estrutura dada por sua segunda forma fundamental é a de $\Sigma$. Além disso, essa imersão é única a menos de isometria de $\mathbb{H}^{2} \times \mathbb{R}$.

Deste resultado, é fácil obter superfícies completas com $K(I)=-1$ em $\mathbb{H}^{2} \times \mathbb{R}$, por exemplo, se tomarmos $h_{u} \equiv 0$ e escolhermos convenientemente $\omega(u, v)=\omega_{1}(u)+\omega_{2}(v)$.

Além disso, já que $h_{u v}=0$ se $h_{u}$ e $h_{v}$ não se anulam então tomando novos parâmetros $(\bar{u}, \bar{v})$ tais que $d \bar{u}=h_{u} d u, d \bar{v}=h_{v} d v$ a equação anterior (4.32) torna-se a equação sinh-Gordon hiperbólica

$$
\omega_{\bar{u} \bar{v}}-\operatorname{senh} \omega \cosh \omega=0 .
$$




\section{Resumo e aplicações}

Aqui apresentaremos os principais resultados vistos nesta dissertação e também veremos algumas aplicações, sem demonstrações, relacionados à estimativas de altura e representação.

\subsection{Resumo}

No capítulo 1, vimos a importante definição de Tensor de Codazzi e de pares de Codazzi.

Vimos as formas assumidas pelas equações para os casos de parâmetros especiais, a saber:

\section{Proposição 1.2.}

Seja $(I, I I) \in \mathcal{P}(\Sigma)$ e z um parâmetro conforme para $I$. Então

$$
\begin{aligned}
I & =2 \lambda|d z|^{2} \\
I I & =q d z^{2}+2 H \lambda|d z|^{2}+\bar{q} d \bar{z}^{2}
\end{aligned}
$$

e se verificam as seguintes equações

$$
\begin{aligned}
K & =H^{2}-\frac{|q|^{2}}{\lambda^{2}}, \\
S \partial_{z} & =H \partial_{z}+\frac{q}{\lambda} \partial_{\bar{z}}, \\
\Gamma_{11}^{1} & =\frac{\lambda_{z}}{\lambda}, \\
\Gamma_{11}^{2} & =0, \\
\Gamma_{12}^{1} & =0, \\
T_{S}\left(\partial_{z}, \partial_{\bar{z}}\right) & =T_{H}\left(\partial_{z}, \partial_{\bar{z}}\right)+\frac{1}{\lambda}\left(\bar{q}_{z} \partial_{z}+q_{\bar{z}} \partial_{\bar{z}}\right), \\
\left\langle T_{S}\left(\partial_{z}, \partial_{\bar{z}}\right), \partial_{z}\right\rangle & =\lambda H_{z}-q_{\bar{z}}, \\
K(I) & =-\frac{1}{\lambda}(\ln \lambda)_{z \bar{z}} .
\end{aligned}
$$


Seja $(I, I I) \in \mathcal{P}(\Sigma)$ onde $I I$ é Riemanniana. Seja $S^{I} \in \mathcal{S}(\Sigma, I I)$ o endomorfismo autoadjunto associado a $I$ com respeito a $I I$ e $S^{I I} \in \mathcal{S}(\Sigma, I)$ o endomorfismo autoadjunto associado a $I I$ com respeito a $I$. Seja $z$ um parâmetro conforme para $I I$, isto é,

$$
\begin{aligned}
I & =p d z^{2}+2 \lambda|d z|^{2}+\bar{p} d \bar{z}^{2}, \\
I I & =2 \rho|d z|^{2},
\end{aligned}
$$

e denotemos

$$
D=|p|^{2}-\lambda^{2}
$$

Então se verificam as seguintes equações

$$
\begin{aligned}
\lambda K & =\rho H \\
S^{I} \partial_{z} & =\frac{H}{K} \partial_{z}+\frac{p}{\rho} \partial \bar{z} \\
S^{I I} \partial_{z} & =K\left(\frac{H}{K} \partial_{z}-\frac{p}{\rho} \partial_{\bar{z}}\right), \\
\Gamma_{11}^{1}+\Gamma_{12}^{2} & =\frac{D_{z}}{2 D} \\
T_{S^{I I}}\left(\partial_{z}, \partial_{\bar{z}}\right) & =\frac{K_{z}}{2} S^{I} \partial_{\bar{z}}-\frac{K_{\bar{z}}}{2} S^{I} \partial_{z}-\frac{(K \bar{p})_{z}}{\rho} \partial_{z}+\frac{(K p)_{\bar{z}}}{\rho} \partial_{\bar{z}} \\
\left\langle T_{S^{I I}}\left(\partial_{z}, \partial_{\bar{z}}\right), \partial_{z}\right\rangle & =2 \rho\left(\frac{K_{z}}{4 K}+\Gamma_{12}^{2}\right) .
\end{aligned}
$$

\section{Proposição 1.4.}

Sejam $(I, I I) \in \mathcal{P}(\Sigma), p \in \Sigma^{\prime}$ e $(u, v)$ parâmetros locais duplamente ortogonais,

$$
\begin{gathered}
I=E d u^{2}+G d v^{2}, \\
I I=k_{1} E d u^{2}+k_{2} G d v^{2} .
\end{gathered}
$$


Então se verificam as seguintes equações

$$
\begin{aligned}
a & =\left(\frac{k_{1}-k_{2}}{2}\right)^{2} \\
\Gamma_{11}^{1} & =\frac{E_{u}}{2 E} \\
\Gamma_{11}^{2} & =-\frac{E_{v}}{2 G}, \\
\Gamma_{12}^{1} & =\frac{E_{v}}{2 E} \\
\Gamma_{12}^{2} & =\frac{G_{u}}{2 G} \\
\Gamma_{22}^{1} & =-\frac{G_{u}}{2 E}, \\
\Gamma_{22}^{2} & =\frac{G_{v}}{2 G}, \\
T_{S}\left(\partial_{u}, \partial_{v}\right) & =T_{H}\left(\partial_{u}, \partial_{v}\right)-\sqrt{a}\left((\ln (\sqrt{a} E))_{u} \partial_{v}+(\ln (\sqrt{q} G))_{v} \partial_{u}\right), \\
\left\langle T_{S}\left(\partial_{u}, \partial_{v}\right), \partial_{u}\right\rangle & =\left(k_{1}\right)_{v}+\sqrt{a} \frac{E_{v}}{E}, \\
\left\langle T_{S}\left(\partial_{u}, \partial_{v}\right), \partial_{v}\right\rangle & =-\left(k_{2}\right)_{u}+\sqrt{a} \frac{G_{u}}{G}, \\
K(I) & =-\frac{1}{2 \sqrt{E G}}\left(\left(\frac{E_{v}}{\sqrt{E G}}\right)_{v}+\left(\frac{E_{u}}{\sqrt{E G}}\right)_{u}\right) .
\end{aligned}
$$

\section{Proposição 1.5.}

Seja $(I, I I) \in \mathcal{P}(\Sigma)$ onde $I I$ é Lorentziana. Suponhamos que existe $S^{I} \in \mathcal{S}(\Sigma, I I)$ endomorfismo autoadjunto associado a $I$ com respeito a $I I$, isto é,

$$
I(X, Y)=I I\left(S^{I} X, Y\right), \quad X, Y \in \mathcal{X}(\Sigma)
$$

e $S^{I I} \in \mathcal{S}(\Sigma, I)$ o endomorfismo autoadjunto associado a $I I$ com respeito a $I$, isto é,

$$
I I(X, Y)=I\left(S^{I I} X, Y\right), \quad X, Y \in \mathcal{X}(\Sigma) .
$$

Seja $(x, y)$ parâmetros assintóticos para $(I, I I)$, isto é

$$
\begin{aligned}
I & =E d x^{2}+2 F d x d y+G d y^{2}, \\
I I & =2 f d x d y .
\end{aligned}
$$

e denotemos por

$$
D=E G-F^{2}
$$


Então se verificam as seguintes equações

$$
\begin{aligned}
F K & =f H \\
S^{I} \partial_{x} & =\frac{H}{K} \partial_{x}+\frac{E}{f} \partial_{y}, \\
S^{I} \partial_{x} & =\frac{G}{f} \partial_{x}+\frac{H}{K} \partial_{y}, \\
S^{I I} \partial_{x} & =K\left(\frac{H}{K} \partial_{x}-\frac{E}{f} \partial_{y}\right), \\
S^{I I} \partial_{y} & =K\left(-\frac{G}{f} \partial_{x}+\frac{H}{K} \partial_{y}\right), \\
\Gamma_{11}^{1}+\Gamma_{12}^{2} & =\frac{D_{x}}{2 D}, \\
\Gamma_{12}^{1}+\Gamma_{22}^{2} & =\frac{D_{y}}{2 D}, \\
T_{S^{I I}}\left(\partial_{x}, \partial_{y}\right) & =\frac{K_{x}}{2} S^{I} \partial_{y}-\frac{K_{y}}{2} S^{I} \partial_{x}-\frac{(K G)_{x}}{f} \partial_{x}+\frac{(K E)_{y}}{f} \partial_{y}, \\
\left\langle T_{S^{I I}}\left(\partial_{x}, \partial_{y}\right), \partial_{x}\right\rangle & =2 f\left(\frac{K_{x}}{4 K}+\Gamma_{12}^{2}\right), \\
\left\langle T_{S^{I I}}\left(\partial_{x}, \partial_{y}\right), \partial_{y}\right\rangle & =-2 f\left(\frac{K_{y}}{4 K}+\Gamma_{12}^{1}\right) .
\end{aligned}
$$

No capítulo 2, vimos a definição e algumas propriedades dos espaços produto, como também as equações necessárias que uma superfície imersa nestes espaços deve satisfazer.

\section{Lema 2.1}

As equações de compatibilidade para uma imersão $\psi$ com curvatura extrínseca positiva pode ser escrita em um parâmetro conforme para a segunda forma fundamental como

$$
\begin{array}{lr}
K(I)=K+\varepsilon \nu^{2}, & \text { Gauss } \\
\frac{\rho_{\bar{z}}}{\rho}+\left(\Gamma_{12}^{1}-\Gamma_{22}^{2}\right)=\varepsilon \alpha \frac{\nu}{\rho}, & \text { Codazzi } \\
h_{z z}=\Gamma_{11}^{1} h_{z}+\Gamma_{11}^{2} h_{\bar{z}}, & \\
h_{z \bar{z}}=\Gamma_{12}^{1} h_{z}+\Gamma_{12}^{2} h_{\bar{z}}+\nu \rho, & \\
\nu_{\bar{z}}=\frac{\alpha K}{\rho} & \\
\frac{1}{D}\left(\alpha h_{z}+\bar{\alpha} h_{\bar{z}}\right)+\nu^{2}=1 . &
\end{array}
$$

No capítulo 3, está presente a estimativa para a altura máxima que pode alcançar um gráfico de curvatura de Gauss constante positiva $K(I)>0$ em $\mathbb{H}^{2} \times \mathbb{R}$ (ou 
$K(I)>1$ em $\left.\mathbb{S}^{2} \times \mathbb{R}\right)$ sobre um plano horizontal, caracterizando, de fato, as superfícies de revolução como aquelas em que se alcança a distância máxima.

\section{Teorema 3.2}

Seja $\psi: \Sigma \rightarrow \mathbb{M}^{2}(\varepsilon) \times \mathbb{R}$ um gráfico compacto sobre um conjunto $\Omega \subset \mathbb{M}^{2}(\varepsilon)$, com curvatura Gaussiana constante positiva $K(I)>\varepsilon$ e cuja fronteira está contida em um slice $\mathbb{M}^{2}(\varepsilon) \times\{0\}$. Então a altura máxima que $\psi$ pode atingir em $\mathbb{M}^{2}(\varepsilon) \times\{0\}$ é

$$
\begin{array}{ll}
\sqrt{\frac{K(I)+1}{K(I)}} \arctan \left(\frac{1}{\sqrt{K(I)}}\right), & \text { se } \quad \varepsilon=-1, \\
\sqrt{\frac{K(I)-1}{K(I)}} \ln \left(\frac{\sqrt{K(I)}+1}{\sqrt{K(I)-1}}\right), & \text { se } \quad \varepsilon=1 .
\end{array}
$$

Além disso, a igualdade ocorre se, e somente se, $\psi$ é o hemisfério de uma $K(I)$-superfície completa.

No capítulo 4, vimos os teoremas de representação para os casos de curvatura extrínseca positiva e curvatura extrínseca negativa.

Para o caso de curvatura extrínseca positiva temos:

\section{Teorema 4.1}

Sejam $\Sigma$ uma superfície e $\psi: \Sigma \rightarrow \mathbb{M}^{2}(\varepsilon) \times \mathbb{R}$ uma $K(I)$-imersão transversa tal que $K>0$. Então, dada um parâmetro local conforme $z$ para $I I$, a primeira e segunda formas fundamentais de $\psi$ podem ser recuperadas em termos de sua função altura $h, \nu$ e a constante $K(I)$ como

$$
\begin{aligned}
& L=\frac{h_{z}^{2}}{1-\nu^{2}}-\frac{\nu_{z}^{2}}{\left(1-\nu^{2}\right)\left(K(I)-\varepsilon \nu^{2}\right)}, \\
& M=\frac{\left|h_{z}\right|^{2}}{1-\nu^{2}}+\frac{\left|\nu_{z}\right|^{2}}{\left(1-\nu^{2}\right)\left(K(I)-\varepsilon \nu^{2}\right)}, \\
& \rho=-\frac{\nu_{z} h_{\bar{z}}+\nu_{\bar{z}} h_{z}}{1-\nu^{2}}>0 .
\end{aligned}
$$

Além disso $h$ e $\nu$ satisfazem

$$
\begin{gathered}
h_{z \bar{z}}=-\frac{(K(I)-\varepsilon) \nu}{\left(1-\nu^{2}\right)\left(K(I)-\varepsilon \nu^{2}\right)}\left(\nu_{z} h_{\bar{z}}+\nu_{\bar{z}} h_{z}\right) \\
\nu_{z \bar{z}}=-\frac{\nu}{\left(1-\nu^{2}\right)\left(K(I)-\varepsilon \nu^{2}\right)}\left(\left(K(I)-2 \varepsilon \nu^{2}+\varepsilon\right)\left|\nu_{z}\right|^{2}\right. \\
\left.\quad+\left(K(I)-\varepsilon \nu^{2}\right)^{2}\left|h_{z}\right|^{2}\right)
\end{gathered}
$$

Reciprocamente, sejam $K(I) \in \mathbb{R}$ e $\varepsilon \in\{-1,1\}$ uma constante, $h: \Sigma \rightarrow \mathbb{R}$ e $\nu: \Sigma \rightarrow(-1,1)$ funções sobre uma superfície de Riemann $\Sigma$ simplesmente conexa 
satisfazendo (5.4) , (5.5), $K(I)-\varepsilon \nu^{2}>0$ e $\nu_{z} h_{\bar{z}}+\nu_{\bar{z}} h_{z}<0$. Então, existe uma imersão $\psi: \Sigma \rightarrow \mathbb{M}^{2}(\varepsilon) \times \mathbb{R}$ tal que $\Sigma$ é uma $K(I)$-superfície transversa cuja primeira e segunda formas são dadas por

$$
\begin{aligned}
I & =L d z^{2}+2 M|d z|^{2}+\bar{L} d \bar{z}^{2}, \\
I I & =2 \rho|d z|^{2},
\end{aligned}
$$

onde $L, M$ e $\rho$ são definidas por (5.1), (5.2) e (5.3), respectivamente; cuja função altura e a quarta coordenada de seu normal são dadas por $h$ e $\nu$, respectivamente; e a estrutura dada por sua segunda forma fundamental é a de $\Sigma$. Além disso, essa imersão é única a menos de isometria de $\mathbb{M}^{2}(\varepsilon) \times \mathbb{R}$.

Para o caso de curvatura extrínseca negativa temos:

\section{Teorema 4.2}

Sejam $\Sigma$ uma superfície conexa e $\psi: \Sigma \rightarrow \mathbb{M}^{2}(\varepsilon) \times \mathbb{R}$ uma $K(I)$-imersão transversa tal que $K<0$. Então, dadas coordenadas assintóticas $(u, v)$ para $I I$, a primeira e segunda formas fundamentais de $\psi$ podem ser recuperadas em termos de sua função altura $h, \nu$ e a constante $K(I)$ como

$$
\begin{aligned}
& E=\frac{h_{u}^{2}}{1-\nu^{2}}-\frac{\nu_{u}^{2}}{\left(1-\nu^{2}\right)\left(K(I)-\varepsilon \nu^{2}\right)}, \\
& F=\frac{h_{u} h_{v}}{1-\nu^{2}}+\frac{\nu_{u} \nu_{v}}{\left(1-\nu^{2}\right)\left(K(I)-\varepsilon \nu^{2}\right)}, \\
& G=\frac{h_{v}^{2}}{1-\nu^{2}}-\frac{\nu_{v}^{2}}{\left(1-\nu^{2}\right)\left(K(I)-\varepsilon \nu^{2}\right)}, \\
& f=-\frac{\nu_{u} h_{v}+\nu_{v} h_{u}}{1-\nu^{2}}>0 .
\end{aligned}
$$

Além disso, $h$ e $\nu$ satisfazem

$$
\begin{gathered}
h_{u v}=-\frac{(K(I)-\varepsilon) \nu}{\left(1-\nu^{2}\right)\left(K(I)-\varepsilon \nu^{2}\right)}\left(\nu_{u} h_{v}+\nu_{v} h_{u}\right) \\
\nu_{u v}=-\frac{\nu}{\left(1-\nu^{2}\right)\left(K(I)-\varepsilon \nu^{2}\right)}\left(\left(K(I)-2 \varepsilon \nu^{2}+\varepsilon\right) \nu_{u} \nu_{v}\right. \\
\left.\quad+\left(K(I)-\varepsilon \nu^{2}\right)^{2} h_{u} h_{v}\right) .
\end{gathered}
$$

Reciprocamente, sejam $K(I) \in \mathbb{R}$ e $\varepsilon \in\{-1,1\}$ constantes, $h: \Sigma \rightarrow \mathbb{R}$ e $\nu: \Sigma \rightarrow(-1,1)$ funções sobre uma superfície de Lorentz simplesmente conexa $\Sigma$ satisfazendo (5.10), (5.11), $K(I)-\varepsilon \nu^{2}<0$ e $\nu_{u} h_{v}+\nu_{v} h_{u}<0$. Então existe uma imersão 
$\psi: \Sigma \rightarrow \mathbb{M}^{2}(\varepsilon) \times \mathbb{R}$ tal que $\Sigma$ é uma $K(I)$-superfície transversa cuja primeira e segunda formas fundamentais são dadas por

$$
\begin{aligned}
I & =E d u^{2}+2 F d u d v+G d v^{2}, \\
I I & =2 f d u d v
\end{aligned}
$$

onde $E, F, G$ e $f$ são definidas por (5.6), (5.7), (5.8) e (5.9), respectivamente; cuja função altura e a quarta coordenada de seu normal são dadas por $h$ e $\nu$, respectivamente; e a estrutura dada por sua segunda forma fundamental é a de $\Sigma$. Além disso, essa imersão é única a menos de isometrias de $\mathbb{M}^{2}(\varepsilon) \times \mathbb{R}$.

\subsection{Aplicações}

Para mostrar a relevância das estimativas de altura e fórmulas de representação, vamos ver nesta seção algumas aplicações destes resultados.

Vejamos, primeiramente, duas aplicações das estimativas de altura.

Em [12], foi utilizada uma estimativa de altura para provar o seguinte resultado:

\section{Proposição 5.1.}

Suponha $M^{2}$ uma superfície compacta sem fronteira cuja curvatura de Gauss é limitada inferiormente por $2 \tau$, para algum número real $\tau$, e suponha $\Sigma$ uma $H$-superfície propriamente mergulhada em $N=M^{2} \times \mathbb{R}$. Se $\tau<0$, assuma que $H^{2} \geq|\tau|$. Então $\Sigma$ não pode estar contida em um semiespaço. Em particular, $\Sigma$ deve ter pelo menos um fim superior $e$ um fim inferior.

Aqui $H$-superfície refere-se a superfícies de curvatura constante. Veja [12] para maiores detalhes.

\section{Teorema 5.1.}

Suponha $\Sigma \subset H_{c}^{3}$ uma superfície completa propriamente mergulhada com curvatura média constante maior do que de uma horoesfera. Então

1. $\Sigma$ não é homeomorfa a uma superfície fechada perfurada em um ponto.

2. Se $\Sigma$ é homeomorfa a uma superfície fechada perfurada em dois pontos, $\Sigma$ é Delaunay. Em particular, $\Sigma$ é topologicamente um cilindro. 
3. Se $\Sigma$ é homeomorfa a superfície fechada perfurada em três pontos, então $\Sigma$ permanece a uma distância limitada de uma geodésica plana de simetria reflexiva. Além disso, cada metade de $\Sigma$ determinada pelo plano de simetria reflexiva é um gráfico sobre este plano com respeito a função distância do plano.

Aqui $H_{c}^{3}$ denota uma forma espacial completa simplesmente conexa tridimensional de curvatura seccional constante negativa $c$. Superfícies Delaunay são superfícies de revolução de curvatura média constante. Veja [13] para maiores informações.

A estimativa de altura apresentada no capítulo 3 tem como hipótese que $\Sigma$ seja um gráfico compacto. Mas, como uma consequência do clássico princípio da reflexão de Alenxandrox para slices em $\mathbb{M}^{2}(\varepsilon) \times \mathbb{R}$ e pelo Teorema (4.2), temos o seguinte corolário:

\section{Corolário 5.1.}

Seja $\psi: \Sigma \rightarrow \mathbb{M}^{2} \times \mathbb{R}$ um mergulho de uma superfície compacta com curvatura Gaussiana constante positiva $K(I)>\varepsilon$, tal que sua fronteira $\psi(\partial \Sigma)$ (possivelmente vazia) está contida em um slice $\mathbb{M}^{2}(\varepsilon) \times\left\{t_{0}\right\}$. Então a diferença da altura entre seu ponto mais alto e seu ponto mais baixo é menor ou igual a

$$
\begin{array}{lr}
2 \sqrt{\frac{K(I)+1}{K(I)}} \arctan \left(\frac{1}{\sqrt{K(I)}}\right) & \text { se } \varepsilon=-1 \\
2 \sqrt{\frac{K(I)+1}{K(I)}} \ln \left(\frac{\sqrt{K(I)}+1}{\sqrt{K(I)-1}}\right) & \text { se } \varepsilon=1 .
\end{array}
$$

Além disso, se uma tal diferença é atingida, $\psi(S)$ é, a menos de isometria, a $K(I)$-superfície completa.

Conforme dissemos na introdução, representações constituem importantes ferramentas, pois garantem a existência de imersões, como também conhecer as expressões para a primeira e a segunda formas fundamentais em termos de algumas funções dadas, se estas funções satisfazem determinadas condições.

Um resultado de representação para superfícies planas pode ser encontrado em [8] em que dada uma $\phi$ harmônica, existe uma superfície plana em $\mathbb{H}^{3}$ parametrizada por linhas de curvatura

$$
\begin{gathered}
I=\cosh ^{2} \phi(u, v)(d u)^{2}+\operatorname{senh}^{2} \phi(u, v)(d v)^{2} \\
I I=\operatorname{senh}^{2} \phi(u, v) \cosh \phi(u, v)\left((d u)^{2}+(d v)^{2}\right) .
\end{gathered}
$$


As parametrizações por linhas de curvatura existem na vizinhança de um ponto não umbílico (para detalhes, seja [15], Teorema 2.4 e Corolário 2.7). Usando este resultado os autores provaram uma caracterização geométrica para uma classes de funções $\phi$, a saber:

\section{Teorema 5.2.}

Um flat front em $\mathbb{H}^{3}$ é helicoidal se, e somente se, há uma parametrização local por linhas de curvatura em um vizinhança de um ponto não singular, tal que a primeira e segunda formas fundamentais são dadas por (5.12) e (5.13), onde

$$
\phi=a u+b v+c \quad e \quad(a, b, c) \neq(0, \pm 1,0) .
$$

Um possível problema pode ser considerado de maneira análoga com o teorema de representação para superfícies em $\mathbb{S}^{2} \times \mathbb{R}$ e $\mathbb{H}^{2} \times \mathbb{R}$, isto é, caracterizar geometricamente superfícies para uma dada classes de soluções para as equações (4.22) e (4.32), que são transformadas em uma classe de equações conhecidas, as equações sinh-Gordon. 


\section{Referências Bibliográficas}

[1] J. A. Aledo, J. M Espinar, J. A. Gálvez, Surfaces with constant curvature in $\mathbb{S}^{2} \times \mathbb{R} e$ $\mathbb{H}^{2} \times \mathbb{R}$. Height estimates and representation, Bulletin fo the Brazilian Mathematical Society, New Series 38(4), (2007), 533 - 554.

[2] J. A. Aledo, J. M. Espinar e J.A. Gálvez, Complete Surfaces of constant curvature in $\mathbb{H}^{2} \times \mathbb{R}$ and $\mathbb{S}^{2} \times \mathbb{R}$, Calc. Var. Partial Differential Equations 29 (2007), no 3, 347 363.

[3] J. A. Aledo, J. M Espinar, J. A. Gálvez, Height estimates for surfaces wiht positive constant mean curvature in $\mathbb{M}^{2} \times \mathbb{R}$, Illinois J. Math 52, (2008), $\mathrm{n}^{\mathrm{o}} 1,203-211$.

[4] B. Daniel, Isometric immersions into $\mathbb{S}^{n} \times \mathbb{R}$ and $\mathbb{H}^{n} \times \mathbb{R}$ and applications to minimal surfaces, Trans. Amer. Math. Soc. 361, (2009), nº 12, 6255-6282.

[5] M. P do Carmo, Geometria Riemanniana, IMPA, 5a ed, Rio de Janeiro (2011).

[6] B. O’Neill, Semi-Reimannian Geometry, Academic Press (1983).

[7] M. P do Carmo, Geometria Diferencial de Curvas e Superfícies, SBM, 5a ed, Rio de Janeiro (2012).

[8] A. Martínez, J. P. dos Santos, K. Tenenblat, Helicoidal Flat Surfaces in Hyperbolic 3-Space, Pacific Journal of Mathematics, (2013), vol. 264, no 1, 195-212.

[9] K. Akutagawa, Harmonic diffeomorphism of the hyperbolic plane, Transactions of the American Mathematical Society, (1994), vol. 342, nº 1, 325-342.

[10] F. Hélein e J. C. Wood, Harmonic maps, Handbook of Global Analysis, (2007).

[11] T. K. Milnor, Abstract Weingarten Surfaces, J. Diff. Geom., 15 (1980), 365-380.

[12] D. Hoffman, J. H. S. de Lira e H. Rosenberg, Constant mean curvature surfaces in $M^{2} \times \mathbb{R}$, Trans. A.M.S., (2006), vol. 358, $\mathrm{n}^{\mathrm{o}} 2$, 491-507.

[13] N. Korevaar, R. Kusner, W. Meeks e B. Solomon, Constant mean curvature surfaces in hyperbolic spaces, Amer. J. Math., 114 (1992), 1-43 
[14] H. Rosenberg e R. Sa Earp, The Geometry of properly embedded special surfaces in $\mathbb{R}^{3}$; e.g., surfaces satisfying $a H+b K=1$, where $a$ and $b$ are positive. Duke Math. J., 73 (1994), no 2, 291-306.

[15] K. Tenenblat, Transformations of manifolds and applications to differential equations, Pitman Monographs and Surveys in Pure and Applied Mathematics 93, Longman, Harlow, 1998.

[16] R. López, Differential geometry of curves and surfaces in Lorentz-Minkowski space, International Electronic Journal of Geometry, 7 (2014), 44-107. 